\title{
Hydrogenation and dehydrogenation of interstellar PAHs: Spectral characteristics and $\mathrm{H}_{2}$ formation
}

\author{
H. Andrews, A. Candian, and A. G. G. M. Tielens
}

Leiden Observatory, Leiden University, PO Box 9513, 2300 RA Leiden, The Netherlands
e-mail: heandrew@strw. leidenuniv.nl

Received 29 April 2016 / Accepted 24 June 2016

\begin{abstract}
Context. We have modelled the abundance distribution and IR emission of the first 3 members of the coronene family in the north-west photodissociation region of the well-studied reflection nebulae NGC 7023.

Aims. Our aim was 3-fold: i) analyze the distribution of abundances; (ii) examine the spectral footprints from the hydrogenation state of polycyclic aromatic hydrocarbons (PAHs); and (iii) assess the role of PAHs in the formation of $\mathrm{H}_{2}$ in photodissociation regions. Methods. To model the physical conditions inside the cloud, we used the Meudon PDR Code, and we gave this as input to our kinetic model. We used specific molecular properties for each $\mathrm{PAH}$, based on the latest data available at the present time. We considered the loss of an $\mathrm{H}$ atom or an $\mathrm{H}_{2}$ molecule as multiphoton processes, and we worked under the premise that PAHs with extra $\mathrm{H}$ atoms can form $\mathrm{H}_{2}$ through an Eley-Rideal abstraction mechanism.

Results. In terms of abundances, we can distinguish clear differences with PAH size. The smallest PAH, coronene $\left(\mathrm{C}_{24} \mathrm{H}_{12}\right)$, is found to be easily destroyed down to the complete loss of all of its $\mathrm{H}$ atoms. The largest species circumcircumcoronene $\left(\mathrm{C}_{96} \mathrm{H}_{24}\right)$, is found in its normal hydrogenated state. The intermediate size molecule, circumcoronene $\left(\mathrm{C}_{54} \mathrm{H}_{18}\right)$, shows an intermediate behaviour with respect to the other two, where partial dehydrogenation is observed inside the cloud. Regarding spectral variations, we find that the emission spectra in NGC 7023 are dominated by the variation in the ionization of the dominant hydrogenation state of each species at each point inside the cloud. It is difficult to "catch" the effect of dehydrogenation in the emitted PAH spectra since, for any conditions, only PAHs within a narrow size range will be susceptible to dehydrogenation, being quickly stripped off of all $\mathrm{H}$ atoms (and may isomerize to cages or fullerenes). The $3 \mu \mathrm{m}$ region is the most sensitive one towards the hydrogenation level of PAHs.

Conclusions. Based on our results, we conclude that PAHs with extra $\mathrm{H}$ atoms are not the carriers of the $3.4 \mu \mathrm{m}$ band observed in NGC 7023, since these species are only found in very benign environments. Finally, concerning the role of PAHs in the formation of $\mathrm{H}_{2}$ in photodissociation regions, we find that $\mathrm{H}_{2}$ abstraction from PAHs with extra $\mathrm{H}$ atoms is an inefficient process compared to grains. Instead, we propose that photodissociation of PAHs of small-to-intermediate sizes could contribute to $\mathrm{H}_{2}$ formation in PDR surfaces, but they cannot account by themselves for the inferred high $\mathrm{H}_{2}$ formation rates in these regions.
\end{abstract}

Key words. astrochemistry - photon-dominated region (PDR) - ISM: molecules - infrared: ISM

\section{Introduction}

Polycyclic aromatic hydrocarbons (PAHs) are a set of molecules consisting of hexagonal aromatic carbon rings, with hydrogen atoms attached at the periphery. Upon absorption of high energy photons they get electronically excited, rapidly redistribute the absorbed energy among all available vibrational states, and relax, either by fragmenting or emitting IR photons through a fluorescence process (Léger \& Puget 1984; Allamandola et al. 1989). Their presence in space is now widely accepted as being the carriers of the strong emission bands at 3.3, 6.2, 7.7, 11.3 and $12.7 \mu \mathrm{m}$, observed in the mid-IR spectra of UV-rich environments such as photodissociation regions (PDRs). PDRs are then considered to be the best laboratories to study PAH emission since they are the UV illuminated surfaces of molecular clouds, where the PAH emission is found to be the strongest.

Observational studies on PDRs using the ISO and Spitzer space telescopes have examined the emission of PAHs in the mid-IR range (Bregman et al. 1989; Berné et al. 2007; Joblin et al. 2010; Boersma et al. 2012, among others). The combination of these studies with theoretical calculations on the IR emission of different PAH species, has allowed a characterization of the emission of PAHs as a whole (Rapacioli et al. 2005; Berné et al. 2007; Rosenberg et al. 2011; Boersma et al. 2013, 2014, 2015; Andrews et al. 2015). Hundreds of theoretical (and experimental) spectra of PAHs have been compiled in the NASA Ames PAH IR Spectroscopic Database (PAHdb ${ }^{1}$; Bauschlicher et al. 2010; Boersma et al. 2014) and the Cagliari PAH Database ${ }^{2}$ (Malloci et al. 2007). The use of such databases has provided great insight on how different classes of PAHs can leave particular footprints in the emission at mid-IR wavelengths. In this regard, spectral variations due to changes in the ionization state of PAH molecules have been well characterized (e.g., Rosenberg et al. 2011; Boersma et al. 2014). Indeed, PAH band ratios such as the 6.2/11.3, 7.7/11.3 and 11.0/11.3 are now used as tracers of the ionization state of the emitting population of PAHs (Galliano et al. 2008; Fleming et al. 2010; Boersma et al. 2014). Likewise, astronomical models have long pointed out the potential importance of hydrogenation for the IR emission spectra (Schutte et al. 1993). Specifically, the $3.4 \mu \mathrm{m}$ band has been interpreted as due to PAHs with extra $\mathrm{H}$ atoms - the so-called, superhydrogenated

\footnotetext{
1 http://www . astrochem.org/pahdb

http://astrochemistry.oa-cagliari.inaf.it/database/
} 
PAHs (Schutte et al. 1993) - in the emitting zones (Sandford et al. 2013; Bernstein et al. 1996) but other explanations for this band have also been put forward, e.g., methylated PAHs and overtones of the $3.3 \mu \mathrm{m} \mathrm{CH}$ stretching mode (Barker et al. 1987; Geballe et al. 1989; Joblin et al. 1996). Observationally, the ratio of the 3.4 to the $3.3 \mu \mathrm{m}$ band is known to vary with distance to the illuminating star (Joblin et al. 1996; Pilleri et al. 2015) and this may provide a test for these assignments.

Over the years, even more sophisticated models have been developed to calculate the IR emission spectra, and to aid in the interpretation of the observed spectral variations. Bakes et al. (2001) developed a model to describe the PAH emission under the physical conditions in the Orion bar, using the latest available data (at that time) on symmetric condensed PAHs up to 54 carbon atoms. That work was the first attempt to study the emission of specific PAH molecules, focusing on the spectral variations produced by changes in the charge distribution of the species. However in PDR environments, PAHs are expected to undergo not only changes in their charge states, but also experience further processing due to the interaction with high energy photons. Allain et al. (1996) developed a kinetic model that considered photodissociation of PAHs. They used generic estimates for the rates of direct $\mathrm{H}$-loss, $\mathrm{H}_{2}$-loss and acetylene-loss, and found that small PAHs ( $<50$ carbon atoms) would be destroyed in highly UV illuminated environments. Based on the available experimental data at that time, Le Page et al. (2001, 2003) modelled the ionization and hydrogenation of generic PAHs (up to 200 carbon atoms) in diffuse clouds in terms of abundances only. Montillaud et al. (2013) built a kinetic model for 4 PAHs up to the size of circumcircumcoronene (96 carbon atoms), and modelled the spatial abundance evolution of these species in the north-west NW PDR of NGC 7023. They only considered neutral and cationic species, and hydrogenation states ranging from the pure carbon clusters up to the parent molecule plus 1 extra hydrogen atom. Their novelty was to admit multiphoton events to describe the photodissociation rates. They showed that multiphoton events can cause the fragmentation of large species, that otherwise would never fragment due to large energies required for such processing.

PAH processing can also leave its impression on the environment. In particular, it has been suggested that the $\mathrm{H}_{2}$ formation rate may be affected by the presence of PAHs, as observations of PDRs have revealed $\mathrm{H}_{2}$ formation rates of up to $10^{-16} \mathrm{~cm}^{3} / \mathrm{s}$ (Habart et al. 2004), that well exceeds the accepted value for diffuse clouds $\left(3 \times 10^{-17} \mathrm{~cm}^{3} / \mathrm{s}\right.$; Jura 1975 ; Gry et al. 2002). For the diffuse medium, $\mathrm{H}_{2}$ is generally thought to form through surface reactions on dust grains (Hollenbach \& Salpeter 1971). Detailed studies have shown that different routes for $\mathrm{H}_{2}$ formation on dust grains are effective in different temperature ranges (e.g., Cuppen \& Herbst 2005; Le Bourlot et al. 2012; Bron et al. 2014). While Langmuir Hinshelwood reactions - where two physisorbed mobile $\mathrm{H}$ atoms interact and desorb as $\mathrm{H}_{2}$ - have been found to be efficient on grains at temperatures below $20 \mathrm{~K}$ (e.g., Pirronello et al. 1997, 1999; Katz et al. 1999), surface reactions involving chemisorbed $\mathrm{H}$ atoms in an Eley-Rideal fashion - where a free $\mathrm{H}$ atom interacts with a chemisorbed $\mathrm{H}$ atom, and desorb as $\mathrm{H}_{2}$ - have been found to be efficient at higher temperatures (e.g., Duley 1996; Habart et al. 2004; Le Bourlot et al. 2012). Surface reactions on PAHs have been proposed as an effective alternative at intermediate temperatures. This is also supported by observational correlations between $\mathrm{H}_{2}$ and $\mathrm{PAH}$ emission in PDRs (Habart et al. 2003, 2004). Experimental and theoretical studies on neutral PAHs with extra $\mathrm{H}$ atoms suggest PAHs could effectively form $\mathrm{H}_{2}$ through Eley-Rideal abstractions
(Bauschlicher 1998; Rauls \& Hornekær 2008; Thrower et al. 2011; Mennella et al. 2012). At the same time, PAHs could also contribute to the $\mathrm{H}_{2}$ budget through photodissociation, where $\mathrm{H}_{2}$-loss competes with $\mathrm{H}$-loss, the main fragmentation channel after photon excitation (e.g., Jochims et al. 1994; Ling et al. 1995). Boschman et al. (2015) built a kinetic model based on the coronene molecule (24 carbon atoms), to study the role of PAHs in the formation of $\mathrm{H}_{2}$ in PDRs. They found that a small PAH like coronene would not form $\mathrm{H}_{2}$ through surface reactions, but mainly through direct loss (i.e., photodissociation). It is of interest then to compare these processes in order to assess the role of PAHs in the formation of $\mathrm{H}_{2}$ in PDRs, and describe how these contributions depend on PAH size.

In this work, we model the abundance distribution of PAHs under the physical conditions expected in a prototypical PDR as the NW PDR of NGC 7023. Our aim is to study the variations in the hydrogenation and ionization state of each species, not only in terms of abundances, but also in terms of their emitted spectra. Extending on previous studies, we are able to make use of the greater availability of theoretical and experimental data on specific PAH species. We focus on the study of PAHs of different sizes that are expected to be relevant in the ISM. Thus, here we intend to extend the work of Bakes et al. (2001) by modelling the PAH emission, taking into account also the hydrogenation degree of each species. We expect then to be able to discern the spectral footprint of the hydrogenation of PAHs. In particular, we would like to assess whether it is feasible for superhydrogenated species to be present in PDRs, and be the carriers for the $3.4 \mu \mathrm{m}$ band whose origin is still unknown (Bernstein et al. 1996; Sandford et al. 2013). Together with this, we will explore the effectiveness of $\mathrm{H}_{2}$ formation on PAHs. For this, we justify our assumptions on the works of Thrower and coworkers, that have proposed $\mathrm{H}_{2}$ abstraction from superhydrogenated PAHs as a feasible pathway for $\mathrm{H}_{2}$ formation (Rauls \& Hornekær 2008; Thrower et al. 2011). Here, we will explore this process for PAHs bigger than coronene, and we will establish how photoprocessing of PAHs can contribute to the formation of $\mathrm{H}_{2}$ in highly UV irradiated environments such as PDRs.

The present work is organized as follows: Sects. 2 and 3 present a general description of all the processes admitted in the model, and how the equations are implemented. The results are presented in Sect. 4 in terms of the variations in the ionization state and hydrogenation state of the PAHs (Sect. 4.1), the $\mathrm{H}_{2}$ formation rate from PAHs (Sect. 4.2), and the spectral variations observed in their emission (Sect. 4.3). The discussion of our results and its implications on the PAH population in the NW PDR of NGC 7023 are presented in Sect. 5.

\section{Physical-chemical processes}

We consider 3 molecules of increasing size within the coronene family: coronene $\mathrm{C}_{24} \mathrm{H}_{12}$, circumcoronene $\mathrm{C}_{54} \mathrm{H}_{18}$, and circumcircumcoronene $\mathrm{C}_{96} \mathrm{H}_{24}$. We chose these molecules since this so-called coronene family is one of the best studied (experimentally and theoretically) set of PAHs. Circumcoronene and circumcircumcoronene consist of a coronene core surrounded by one and two rings of hexagons respectively (see Fig. 1). Because of their similarity in molecular structure, their physical and spectroscopic properties show a global trend (Ricca et al. 2012). The sizes of these three species span the range of astrophysical relevant PAHs (Allamandola et al. 1989; Croiset et al. 2016). Also, given their compact structure, the coronene 
Coronene
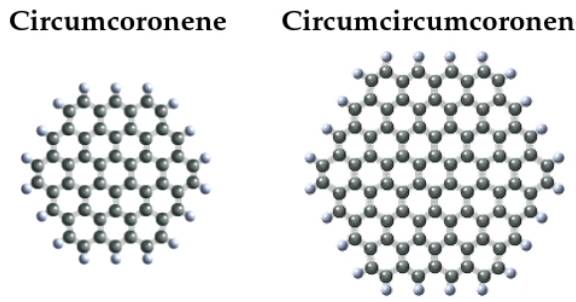

Fig. 1. PAHs studied in this work. From left to right: coronene $\left(\mathrm{C}_{24} \mathrm{H}_{12}\right)$, circumcoronene $\left(\mathrm{C}_{54} \mathrm{H}_{18}\right)$ and circumcircumcoronene $\left(\mathrm{C}_{96} \mathrm{H}_{24}\right)$. The carbon cores are depicted in grey, while $\mathrm{H}$ atoms are depicted in white.

family is expected to be among the most stable PAHs in the ISM (Ricca et al. 2012).

PAHs in our model absorb photon energies up to $E_{\max }=$ $13.6 \mathrm{eV}$, as expected in PDRs. All possible ionization states (given this threshold energy) are admitted in our model. In terms of hydrogenation state, we refer to the parent molecule as the molecule with a number of hydrogen atoms $N_{\mathrm{H}}$ equal to $N_{\mathrm{H}}^{0}$, where $N_{\mathrm{H}}^{0}$ corresponds to 12 for coronene, 18 for circumcoronene, and 24 for circumcircumcoronene. Parent molecules will also be referred to as the molecule in the normal hydrogenated state. All molecules with $N_{\mathrm{H}}<N_{\mathrm{H}}^{0}$ are referred to as dehydrogenated derivatives; while molecules with extra hydrogen atoms $N_{\mathrm{H}}>N_{\mathrm{H}}^{0}$ are denominated as molecules in superhydrogenated states (Schutte et al. 1993). Throughout this work we consider that the hydrogenation state of each molecule can go from being completely dehydrogenated $\left(N_{\mathrm{H}}=0\right)$ to having up to 4 additional $\mathrm{H}$ atoms $\left(N_{\mathrm{H}}=N_{\mathrm{H}}^{0}+4\right)$. A discussion on this choice will be given later in the paper (see Sect. 5). In the following, we will describe all the parameters and assumptions regarding the processes considered in our model.

\subsection{Environment}

We analyze the PAH abundance and emission spectra in the environment of the northwest NW PDR of the well-studied reflection nebula NGC 7023 (RA (J 2000.0): 2101 32.3; Dec (J 2000.0): +68 10 25.4). Figure 2 shows the region studied in this work. The NW PDR of NGC 7023 is located $\sim 45^{\prime \prime}$ from the exciting binary system HD 200775 (Alecian et al. 2008), and it is the brightest one in the mid-IR extending to about 60" (Werner et al. 2004). It is often considered as a prototype of a PDR due to its edgeon structure, which shows a clear stratification of the emitting region (Pilleri et al. 2012). The star has created a low-density cavity that is surrounded by a dense molecular cloud apparent in ${ }^{12} \mathrm{CO},{ }^{13} \mathrm{CO}, \mathrm{CO}^{+}$and other species (Fuente et al. 1998, 2003). The cavity edge is lined by a layer of HI (Fuente et al. 1998). The molecular cloud surface is traced by fluorescent $\mathrm{H}_{2}$ emission and by bright Extended Red Emission (Lemaire et al. 1996; Witt et al. 2006). Spitzer/IRS observations have revealed the presence of $\mathrm{C}_{60}$ in this nebula (Sellgren et al. 2010; Berné \& Tielens 2012; Berné et al. 2015). While the PAH abundance decreases rapidly into the cavity, the fullerene abundance actually increases (Berné $\&$ Tielens 2012). The average size of the PAHs at the PDR front is smaller than the sizes of PAHs deeper in the PDR, as well as the PAHs in the cavity (Croiset et al. 2016). All of these effects have been attributed to photoprocessing of PAHs, driving dehydrogenation, graphene formation and isomerization to cages and fullerenes (Berné \& Tielens 2012; Berné et al. 2015).

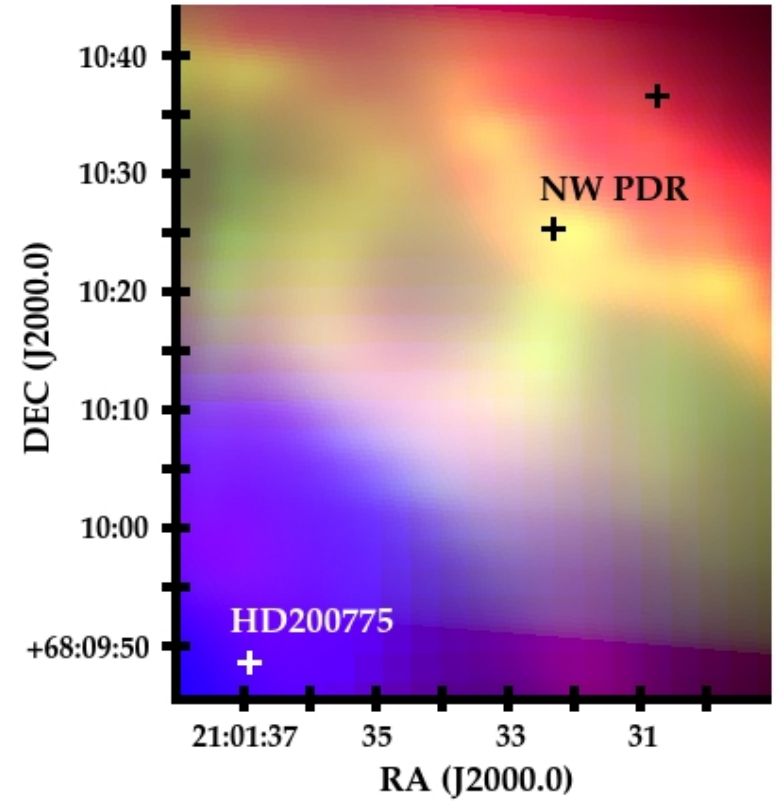

Fig. 2. Composite image of the NW PDR of NGC 7023. The threecolor image combines the PACS $70 \mu \mathrm{m}$ (red), PAH emission at $6.2 \mu \mathrm{m}$ (green, image courtesy of O. Berné), and MIPS $24 \mu \mathrm{m}$ (blue) images. The white cross indicates the position of the star HD 200775, while the black crosses indicate the reference extension of the NW PDR (at $45^{\prime \prime}$ and $60^{\prime \prime}$ away from the star).

Table 1. Input parameters for the modelling of the NW PDR of NGC 7023 using the Meudon PDR Code.

\begin{tabular}{cc}
\hline \hline Property & Values \\
\hline Initial density $n_{\mathrm{H}}\left(\mathrm{cm}^{-3}\right)$ & $2 \times 10^{4}$ \\
UV field $G_{0}$ (Habing units) & 2600 \\
Cosmic rays ionization rate $\left(\mathrm{s}^{-1}\right)$ & $5 \times 10^{-17}$ \\
Pressure $\left(\mathrm{cm}^{-3} \mathrm{~K}\right)$ & $7 \times 10^{6}$ \\
$R_{V}$ & 5.56 \\
$\mathrm{~N}_{\mathrm{H}} / E(B-V)$ & $5.8 \times 10^{21}$ \\
Grains to gas mass ratio & 0.01 \\
Grains distribution index & 3.5 \\
Grains minimum radius $(\mathrm{cm})$ & $3 \times 10^{-7}$ \\
Grains maximum radius $(\mathrm{cm})$ & $3 \times 10^{-5}$ \\
\hline
\end{tabular}

\subsection{PDR model}

In order to model the physical conditions within the PDR region we use the Meudon PDR Code (version 1.4.4; Le Petit et al. 2006; Le Bourlot et al. 2012). This code has been widely used to describe the NW PDR region (see Joblin et al. 2010; Montillaud et al. 2013; Bernard-Salas et al. 2015, among others). It calculates the steady state structure, solving the radiation transfer equation and the thermal and chemical balance as a function of depth in each plane-parallel slab of the cloud.

Table 1 lists the input parameters we adopted from the literature. We consider an isobaric model following Montillaud et al. (2013). As suggested by the Meudon PDR Code guide, we consider a cloud of finite size $\left(A_{V_{\max }}=5\right)$ being illuminated by the stellar radiation on one side, and a radiation field of 

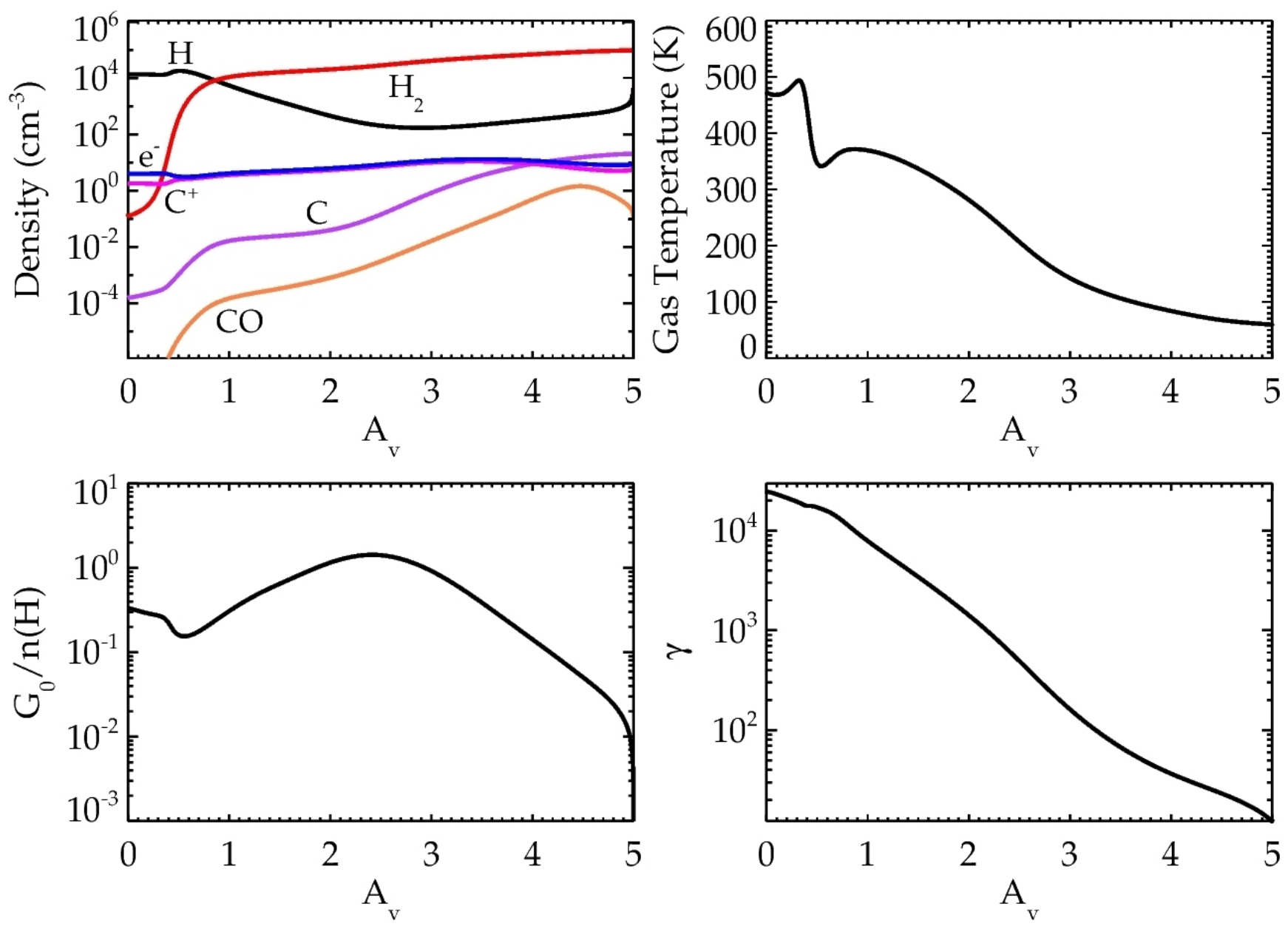

Fig. 3. Physical conditions in the NW PDR of NGC 7023. The top panels show the variation of the densities and gas temperature inside the cloud. The H I to $\mathrm{H}_{2}$ transition occurs at an $A_{V} \sim 0.9$, while the C II-C I transition occurs deeper in the cloud at an $A_{V} \sim 4$. The bottom panels show the physical parameters relevant for PAH hydrogenation and ionization evolution. The hydrogenation of PAHs is expected to be controlled by the ratio between the UV field intensity $G_{0}$ and the density of atomic hydrogen, which in our case only varies by about 2 orders of magnitude throughout the cloud. The ionization state of the PAH population on the other hand depends on the so-called ionization parameter, $\gamma \equiv G_{0} \sqrt{T_{\text {gas }}} / n(e)$, which measures the ratio between ionization and electron recombination of the PAH species.

standard intensity on the other side. Analysis of the observed farIR continuum intensity yields an incident UV field of $G_{0}=2600$ Habing field units in the region of interest (Chokshi et al. 1988; Pilleri et al. 2012). We consider the flux of a binary star with both components modelled with a Kurucz stellar spectrum of $15000 \mathrm{~K}$ each (Kurucz 1993; Pilleri et al. 2012). Young Owl et al. (2002) estimated a gas density of $4 \times 10^{3} \mathrm{~cm}^{-3}$ from the observed line intensities of [O I] $63 \mu \mathrm{m}$ and [C II] $158 \mu \mathrm{m}$, which is in good agreement with H I observations from Fuente et al. (1998). More recent observations of the cooling lines with Herschel have led to slightly higher density estimates of the region $\left(10^{4} \mathrm{~cm}^{-3}\right.$; Bernard-Salas et al. 2015). SPIRE observations of the $\mathrm{CO}$ rotational lines, plus an analysis of the dust continuum emission show the inhomogeneous structure of the NW PDR of NGC 7023, leading to density estimates as high as $10^{5} \mathrm{~cm}^{-3}$ in the filaments and at large distances from the PDR front (Köhler et al. 2014). Here, we adopt a value of $2 \times 10^{4} \mathrm{~cm}^{-3}$ as the initial density (Pilleri et al. 2012).

Figure 3 shows the calculated physical conditions. At the surface of the cloud, hydrogen is mainly in atomic form, and the temperature of the gas is a few hundred $\mathrm{K}$. The conversion from $\mathrm{HI}$ to $\mathrm{H}_{2}$ occurs at an extinction of $A_{V}=0.9$. The density of the gas increases towards high $A_{V}$ reaching $\sim 10^{5} \mathrm{~cm}^{-3}$, while the gas temperature decreases, reaching values comparable to the dust temperature $\left(T_{\text {gas }} \sim 60 \mathrm{~K}\right)$. Going into the PDR, the abundance of electrons decreases slightly due to neutralization reactions with atomic species, but from $A_{V}=0.4$ onwards, the electron abundance follows closely that of ionized carbon. Carbon is mostly in ionized form. The conversion from C II to C I occurs at an $A_{V} \sim 4$. The $\mathrm{CO}$ abundance increases with depth reaching a maximum at $A_{V} \sim 4.5$, from where it starts dissociating due to photons penetrating from the other side of the cloud.

The physics of PAHs in PDRs is controlled by two parameters: the ionization parameter, $\gamma \equiv G_{0} \sqrt{T_{\text {gas }}} / n(e)$, which controls the ionization balance of PAHs (Bakes \& Tielens 1994), and the hydrogenation parameter, $G_{0} / n(\mathrm{H})$, which sets their level of dehydrogenation (Tielens 2005). The variation of these parameters in the NW PDR of NGC 7023 is shown in Fig. 3. The ionization parameter decreases by several orders of magnitude into the PDR due to the attenuation of the UV radiation field, coupled with the relatively constant electron density for this isobaric model. In contrast, the variations in the hydrogenation parameter are more modest, but also show a more complex behaviour as the UV field and the hydrogen abundance decrease inwards but not in lockstep. Starting at the surface of the cloud, as $A_{V}$ increases, $G_{0} / n(\mathrm{H})$ decreases due to the rapid attenuation of the radiation 

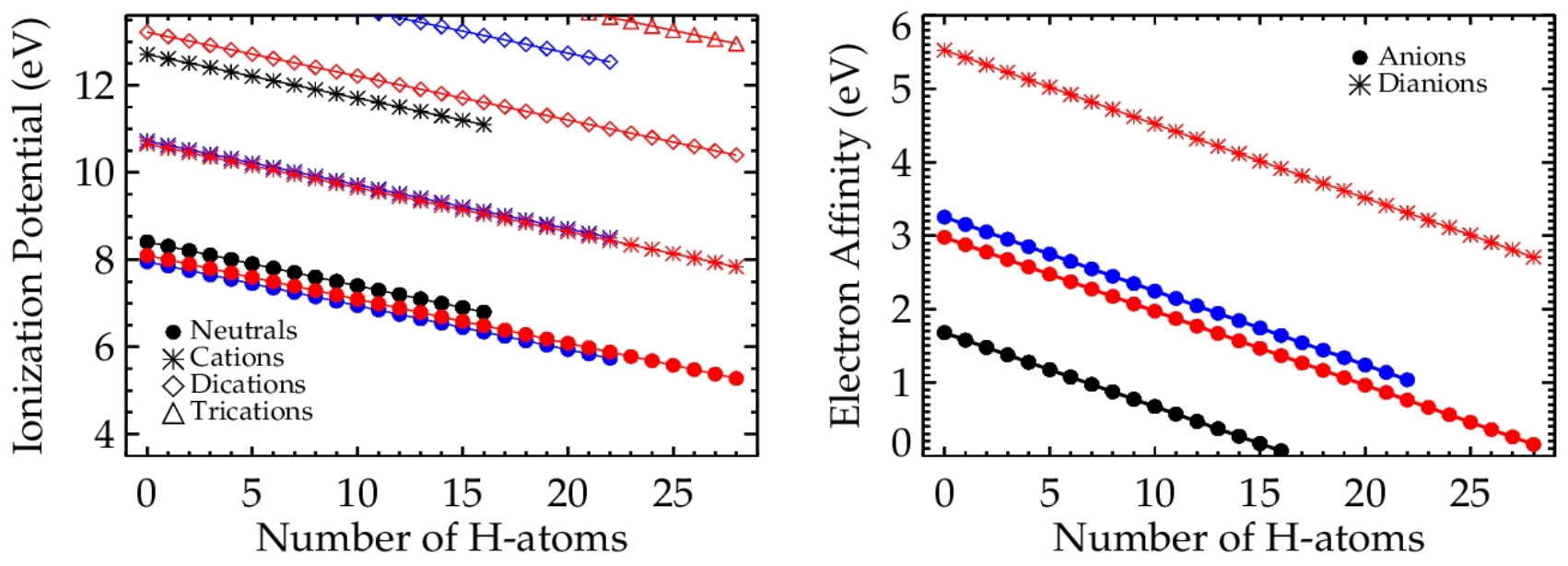

Fig. 4. Ionization potentials and electron affinities assumed in our model. Energies for coronene, circumcoronene and circumcircumcoronene derivatives are shown in black, blue and red, respectively. Given that PAHs in our model can absorb photon energies up to $13.6 \mathrm{eV}$, the highest charge state for coronene derivatives is the doubly charged one $(Z=2)$. Circumcoronene derivatives can be doubly ionized, and only those having more than $12 \mathrm{H}$ atoms can be triply ionized $(Z=3)$. Circumcircumcoronene derivatives on the other hand can be triply ionized, and those having more than $22 \mathrm{H}$ atoms can be found in the +4 charge state. Given the uncertainty in the electron affinities for highly negative charge states $(Z<-1)$, we do not take into account that the more dehydrogenated molecules could also exist in these highly negative states (e.g., $Z=-2$ for circumcoronene derivatives).

field compared to the density, which stays relatively constant until the $\mathrm{HI}$ to $\mathrm{H}_{2}$ transition occurs. Once this point is reached, the decrease in the atomic hydrogen density translates into an increase of $G_{0} / n(\mathrm{H})$ to a maximum value of 1.43 at an $A_{V} \sim 2.4$. After this point, $G_{0} / n(\mathrm{H})$ rapidly decreases as the UV field attenuates while the hydrogen density hardly changes.

\subsection{Molecular characteristics}

When absorbing a UV photon, PAHs in our model can either ionize or dissociate through the loss of an $\mathrm{H}$ atom or an $\mathrm{H}_{2}$ molecule, as long as the absorbed energy is large enough to open these channels and win over IR relaxation. It is important to mention that we do not consider $\mathrm{C}$ nor $\mathrm{C}_{2} \mathrm{H}_{2}$-losses, as the kinetics of these processes are rather uncertain. Experiments have shown that, even for small PAHs, H-loss dominates over C-loss (Jochims et al. 1994). For PAHs larger than some $30 \mathrm{C}$ atoms, $\mathrm{UV}$ photolysis proceeds by stripping off all $\mathrm{H}$ atoms before C-loss sets in (Zhen et al. 2014a,b; Castellanos et al., in prep.). This separates photo fragmentation into two processes where in the first step, the H-coverage of PAHs is set by a balance of UV driven $\mathrm{H}$-loss and hydrogenation reactions, controlled by the hydrogenation parameter, $G_{0} / n(\mathrm{H})$. Only when all $\mathrm{H}$ atoms are lost, will C-loss commence and this will occur close to the star. In our analysis, we have ignored this second step. The separation of photolysis into two distinct steps simplifies the model analysis considerably, as only a limited number of species needs to be followed.

Since the internal energies required for fragmentation of large PAHs to be competitive with IR relaxation are very high (e.g., $15 \mathrm{eV}$ for $\mathrm{C}_{54} \mathrm{H}_{18}$ ), we consider photodissociation as a multiphoton process where PAHs can absorb more than one photon before fully cooling down. Details on how we describe the photodissociation rates are given in Sect. 2.4.3.

Superhydrogenated species are allowed to form $\mathrm{H}_{2}$ through Eley-Rideal abstraction. We also consider attachment/recombination with electrons and $\mathrm{H}$-addition reactions. $\mathrm{H}_{2}$-addition is not considered as experimental data indicates that such reactions are not important in PDRs.
In the following we will describe the molecular characteristics we consider in order to calculate the kinetic rates for all the processes taken into account in our work. The mathematical formulation of each rate will be given in Sect. 2.4.

\subsubsection{Ionization potentials and electron affinities}

We use specific ionization potentials (IP) and electron affinities (EA) for each of the molecules considered. Given the photon energies available $(<13.6 \mathrm{eV})$, we have to consider multiple cationic states. For coronene and circumcoronene we only have to consider the anionic state $(Z=-1)$ but for circumcircumcoronene we have to consider dianions as well $(Z=-2)$. For the normally hydrogenated species, we have taken estimates from experiments when available (Tobita et al. 1994). If no experimental values were available, we then adopted results from quantum theory (Malloci et al. 2007, 2008), or estimates based upon conducting disk behaviour (Bakes \& Tielens 1994). See more details in Appendix A.

For dehydrogenated PAHs, we have followed Malloci et al. (2008) who calculated the IPs for the even dehydrogenated states of coronene, i.e., $\mathrm{C}_{24} \mathrm{H}_{2 n}$ where $n=0-6$. Based on their estimated change of IP with hydrogenation level of the PAH, $\triangle \mathrm{IP}$, we assume that for each PAH in charge state $Z$, and with $N_{\mathrm{H}}$ number of $\mathrm{H}$ atoms, the following relation applies:

$\operatorname{IP}\left(Z, N_{\mathrm{H}}\right)=\operatorname{IP}\left(Z, N_{\mathrm{H}}^{0}\right)+\Delta \operatorname{IP} \times\left(N_{\mathrm{H}}^{0}-N_{\mathrm{H}}\right)$

where $\operatorname{IP}\left(Z, N_{\mathrm{H}}^{0}\right)$ is the IP of the parent molecule in charge state $Z$, and $\triangle \mathrm{IP}$ is constant $(\sim 0.1 \mathrm{eV}$; see Fig. 4$)$. We adopt this for all hydrogenation states allowed (see more in Appendix A).

Regarding EAs, the first EA of $\mathrm{C}_{24} \mathrm{H}_{12}$ is $0.47 \mathrm{eV}$, as derived from both theory and experiments (Duncan et al. 1999; Malloci et al. 2007). An et al. (2008) reports an EA of $1.89 \mathrm{eV}$ for $\mathrm{C}_{24}$, which agrees with the $\sim 0.1 \mathrm{eV}$ variation per dehydrogenation derived from the IPs. Therefore, we also consider the relation given by Eq. (1) for the EAs (see Fig. 4). 


\subsubsection{Ionization yield and UV absorption cross sections}

We adopt the ionization yield, $Y_{\text {ion }}$, derived from experiments on small PAHs from Jochims et al. (1996; see also Jochims et al. 1997). These experiments show that the ionization yield scales linearly with energy, until it reaches unity for energies above $\sim(\mathrm{IP}+9.2) \mathrm{eV}$. This describes well the ionization yield measured for coronene (Verstraete et al. 1990), supporting the fact that the variation of the ionization yield with energy appears to be rather independent of the size of the PAH.

We have adopted the UV absorption cross sections, $\sigma_{\mathrm{UV}}$, from the Cagliari PAH database for $\mathrm{C}_{24} \mathrm{H}_{12}$ and $\mathrm{C}_{54} \mathrm{H}_{18}$ (Malloci et al. 2007). For $\mathrm{C}_{96} \mathrm{H}_{24}$ no information is available in the literature, and so we adopt the UV cross sections of $\mathrm{C}_{54} \mathrm{H}_{18}$ scaled by the number of carbon atoms of circumcircumcoronene.

For the even dehydrogenated states of coronene, we use the DFT results from Malloci et al. (2008). For the other species we use the UV absorption cross section of the molecule with the closest number of $\mathrm{H}$ atoms within the same ionization state. We expect this to be a good approximation, based on the analysis of Malloci et al. (2008). Even though the overall shape varies somewhat with dehydrogenation, when calculating the average photon energy absorbed in a given stellar radiation field, the absorbed average energy varies by less than $1 \mathrm{eV}$ for the different hydrogenation states, being slightly higher for dehydrogenated states. Thus, we expect that this assumption will have little impact on our calculations.

\subsubsection{IR cross sections and density of states}

IR cross sections, $\sigma_{\bar{v}_{i}}$, and frequencies, $\bar{v}_{i}$ were collected either from the NASA Ames PAH Database (PAHdb; Bauschlicher et al. 2010; Boersma et al. 2014), or from our own ab initio DFT calculations, using B3LYP/4-31G (Becke 1993; Stephens et al. 1994) on Gaussian 09 (Frisch et al. 2009). In the case of dehydrogenated PAHs, several isomers with various multiplicities were calculated. To facilitate our analysis, here we always report the vibrational spectrum of the most stable isomer. Following Langhoff (1996) the vibrational frequencies were scaled by 0.958 , except for the modes involving CC triple bonds. Bauschlicher \& Ricca (2013) showed that B3LYP/4-31G failed to reproduce the stretching modes of the triple $\mathrm{CC}$ bond in $\mathrm{C}_{6} \mathrm{H}_{4}$. In order to compensate for this, a specific scaling factor of 0.9097 was used. This was obtained by direct comparison of the calculated frequency for the triple $\mathrm{CC}$ bond stretching mode of $\mathrm{C}_{6} \mathrm{H}_{4}$, with the experimental result published in Radziszewski et al. (1992).

Tables summarizing the set of molecules for which IR spectra are available can be found in Appendix A. For the molecules for which we have the intrinsic spectra, density of vibrational states are calculated explicitly using the Beyer-Swinehart counting algorithm (Beyer \& Swinehart 1973; Stein \& Rabinovitch 1973). Whenever this data is not available, we consider the spectra of the molecule with the closest number of $\mathrm{H}$ atoms within the same ionization state.

\subsection{Processes}

In this section we specify the processes for each molecule, and we describe how we calculate each rate using the parameters described in Sect. 2.3.

\subsubsection{Photoionization}

The photoionization rate, $k_{\text {ion }}$, for a molecule in a given ionization state, $Z$, and with $N_{\mathrm{H}} \mathrm{H}$ atoms is given by:

$k_{\text {ion }}\left(Z, N_{\mathrm{H}}\right)=\int_{\mathrm{IP}\left(Z, N_{\mathrm{H}}\right)}^{E_{\max }} \sigma_{\mathrm{UV}}\left(Z, N_{\mathrm{H}}, E\right) Y_{\mathrm{ion}}\left(Z, N_{\mathrm{H}}, E\right) n(E) \mathrm{d} E$,

where $\sigma_{\mathrm{UV}}\left(Z, N_{\mathrm{H}}\right)$ is the $\mathrm{UV}$ absorption cross section of the molecule, $Y_{\text {ion }}\left(Z, N_{\mathrm{H}}\right)$ is the ionization yield, and $n(E)$ corresponds to the flux of photons.

\subsubsection{Electron attachment/recombination with electrons}

We have adopted electron attachment and recombination rates based on the collisional rates described in Bakes \& Tielens (1994), which are in good agreement with experiments for small PAHs (see Appendix A for details).

For the coronene anion formation our estimate gives a value of $\sim 3 \times 10^{-10} \mathrm{~cm}^{3} / \mathrm{s}$, which is slightly smaller than the rate coefficient determined by Denifl et al. (2005). With respect to the measured rates for small cationic PAHs, the experimental values are smaller than our formalism would predict, but they seem to be approaching our adopted conducting disk values with increasing size (Tielens 2008).

Note that electron recombination is an excitation process and can lead to fragmentation if the dissociative channel is energetically accessible (Tobita et al. 1992; Denifl et al. 2005). Therefore, we explored direct $\mathrm{H}$-loss and $\mathrm{H}_{2}$-loss as possible pathways for relaxation after recombination with an electron. However, we found that these channels are, in general, not relevant for the molecules considered. Only for coronene derivatives we find that dissociative recombination can be important for superhydrogenated cations. However, as we will see in Sect. 4, these are not relevant derivatives of coronene.

\subsubsection{Photodissociation channels}

As mentioned before, we consider dissociation and IR emission as possible relaxation channels after the absorption of nonionizing photons. We calculate the IR emission rate explicitly using either the IR cross sections of the respective molecule if available, or the IR cross sections from the molecule with the closest number of $\mathrm{H}$ atoms, within the same ionization state. Table 2 shows the adopted parameters for each photodissociation process. The explanation of each choice is given below.

$\mathrm{H}$-loss. For coronene and its dehydrogenated states $\left(N_{\mathrm{H}} \leq 12\right)$, we will use the activation energies and entropies derived for pyrene cation from the time-dependent mass spectrometry experiments of Ling et al. (1995); that is, activation energies of 4.6 and $4.1 \mathrm{eV}$, and entropies of 44.8 and $55.6 \mathrm{~J} / \mathrm{K} / \mathrm{mol}$, for PAHs with an even and odd number of $\mathrm{H}$ atoms, respectively (see Table 2).

These values compare well with our theoretically determined dissociation energies for the coronene cation $(Z=1)$ and its dehydrogenated states (4.9 and $3.9 \mathrm{eV}$ respectively), as well as with Reitsma et al. (2014) for the first $6 \mathrm{H}$-losses $(4.7$ and $4.1 \mathrm{eV}$ respectively). Theoretical analysis also shows that the $\mathrm{CH}$ interaction is a rather local process, and thus, its energetics remain relatively constant with increasing degree of dehydrogenation of the molecule: considering even and odd dehydrogenated states separately, only small variations of $\sim 0.02 \mathrm{eV}$ are obtained 
Table 2. Dissociation parameters considered in our work.

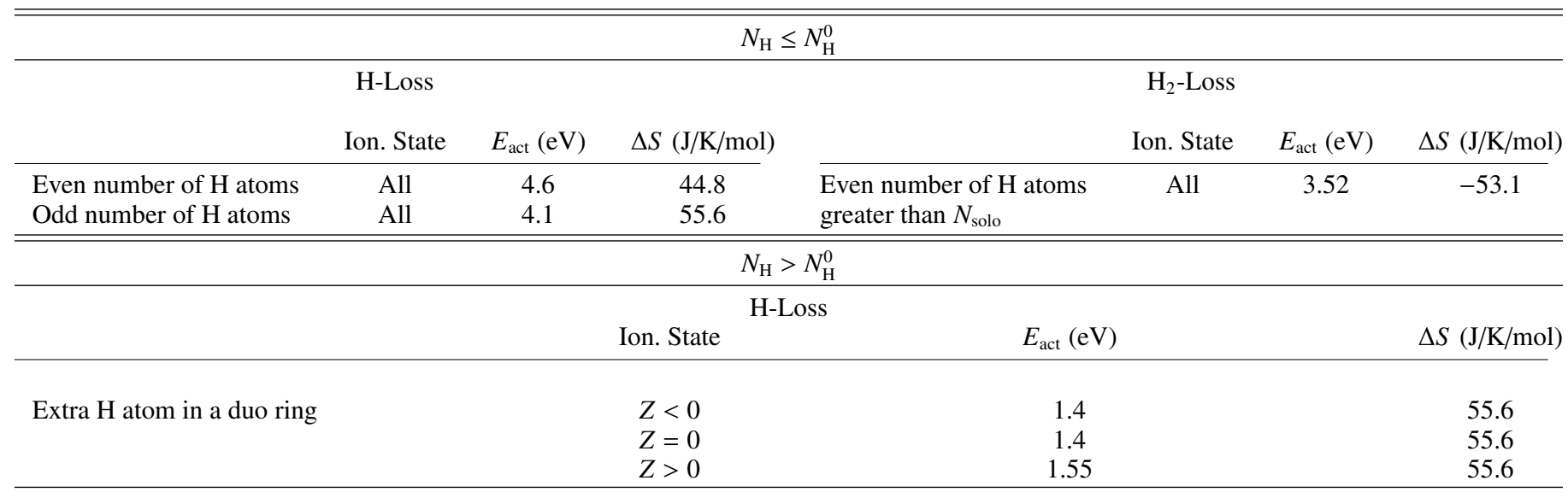

Notes. The upper table shows the activation energies, $E_{\text {act }}$, and change in entropy, $\Delta S$, for the normal-to-dehydrogenated states $\left(N_{\mathrm{H}} \leq N_{\mathrm{H}}^{0}\right)$, while the bottom table shows the dissociation parameters for the superhydrogenated states $\left(N_{\mathrm{H}}>N_{\mathrm{H}}^{0}\right)$. $N_{\text {solo }}$ corresponds to the number of solo $\mathrm{H}$ atoms of each molecule, i.e., 0 for coronene, 6 for circumcoronene and 12 for circumcircumcoronene. Dissociation parameters for normal-todehydrogenated molecules are taken from Ling et al. (1995). The activation energies considered for the H-loss from superhydrogenated molecules are taken from the theoretical work of Bauschlicher \& Ricca (2014). These corresponds to the energies required to remove the extra $\mathrm{H}$ atom in a duo ring.

(Candian et al., in prep.). Calculations also predict little difference $(<0.4 \mathrm{eV})$ in the energies between neutral and positively ionized coronene (Paris et al. 2014); and also between their respective neutral and positively ionized dehydrogenated states (Candian et al., in prep.). Concerning anionic species, the energy for the first $\mathrm{H}$-loss from coronene anion is about $4.1 \mathrm{eV}$ according to DFT calculations (Candian et al., in prep.), much less than the $4.9 \mathrm{eV}$ obtained for the other ionization states of coronene. However, calculations on anions require a different level of theory which may add a systematic difference with respect to the values obtained for the other ionization states. Keeping this in mind, we will assume the same energies and entropies for all ionization states.

For $\mathrm{C}_{54} \mathrm{H}_{18}$ and $\mathrm{C}_{96} \mathrm{H}_{24}$, and their respective dehydrogenated states $\left(N_{\mathrm{H}} \leq N_{\mathrm{H}}^{0}\right)$, we must take into account their edge structure in order to define how these molecules fragment (see Fig. 1). While coronene has a carbon core with $12 \mathrm{H}$ atoms attached in pairs to 6 different rings (i.e., 6 duo rings), $\mathrm{C}_{54} \mathrm{H}_{18}$ has $12 \mathrm{H}$ atoms attached in pairs to 6 separate rings (6 duo rings), and $6 \mathrm{H}$ atoms attached to the other 6 peripheral rings (6 solo rings). Circumcircumcoronene has similarly 6 duo rings and 12 solo rings. Given these structures, we will consider that these molecules first lose all the duo $\mathrm{H}$-atoms in the same fashion as the $\mathrm{H}$-losses in coronene. Once the duo $\mathrm{H}$-atoms are gone, then they start losing the solo H-atoms. This assumption is based on theoretical calculations that predict higher binding energies for solo $\mathrm{H}$-atoms than duo $\mathrm{H}$-atoms (Castellanos et al., in prep.)

For the loss of the extra hydrogen atoms (i.e., $N_{\mathrm{H}}>N_{\mathrm{H}}^{0}$ ) we adopt the theoretically derived values from Bauschlicher \& Ricca (2014) for the binding energies of the extra H-atom for the $\mathrm{C}_{96} \mathrm{H}_{25}$ anion, neutral and cation molecule. We will assume extra $\mathrm{H}$ atoms always stick to duo positions, even for $\mathrm{C}_{54} \mathrm{H}_{18}$ and $\mathrm{C}_{96} \mathrm{H}_{24}$ derivatives that also have solo rings available for attachment. The binding energies of an extra $\mathrm{H}$-atom in a duo position are of $1.4 \mathrm{eV}$ for the anion, $1.4 \mathrm{eV}$ for the neutral and $1.55 \mathrm{eV}$ for the cation. The respective energies for the attachment to a solo position are of 2.2, 1.8 and $2.3 \mathrm{eV}$. These small differences do not have an impact on the derived rates. Given the lack of data, we will use an activation entropy of $55.6 \mathrm{~J} / \mathrm{K} / \mathrm{mol}$, as assumed for PAHs with an odd number of $\mathrm{H}$ atoms (Ling et al. 1995).

$\mathrm{H}_{2}$-loss. The loss of $\mathrm{H}_{2}$ through photolysis will be considered for normally-to-dehydrogenated molecules, while $\mathrm{H}_{2}$-loss from superhydrogenated molecules will be considered as an abstraction process through an Eley-Rideal mechanism. Test runs show that for superhydrogenated molecules, $\mathrm{H}_{2}$ abstraction by $\mathrm{H}$ is more important than $\mathrm{H}_{2}$-loss through photoexcitation. According to DFT calculations (Castellanos et al., in prep.), the activation energies for the $\mathrm{H}_{2}$-loss from a duo and a solo position are of 3.89 and $2.2 \mathrm{eV}$, respectively. Assuming a change in entropy of $-53.1 \mathrm{~J} / \mathrm{K} / \mathrm{mol}$ (Ling et al. 1995), we get that superhydrogenated molecules will preferably lose their extra $\mathrm{H}$ atom after UV photon absorption, rather than an $\mathrm{H}_{2}$ molecule, and that superhydrogenated isomers with free $\mathrm{C}$ atoms are not relevant species. Thus, we do not consider direct $\mathrm{H}_{2}$-loss from superhydrogenated molecules.

Regarding normally-to-dehydrogenated molecules, due to the lack of experimental data on large PAHs, we will use the activation energy and entropy obtained for pyrene cation from the experiments of Ling et al. (1995); that is, an activation energy of $3.52 \mathrm{eV}$, and an activation entropy of $-53.1 \mathrm{~J} / \mathrm{K} / \mathrm{mol}$. This energy is small compared to the theoretical calculations for the first $\mathrm{H}_{2}$-losses from coronene cation, which give energies of $\sim 5.0 \mathrm{eV}$ (Reitsma et al. 2014; Paris et al. 2014). However DFT calculations are known to overestimate energies, and so we chose to use the experimental values.

A theoretical study on coronene and its positively ionized states shows that $\mathrm{H}_{2}$-loss from $2 \mathrm{H}$ atoms within the same ring has a lower dissociation energy than the $\mathrm{H}_{2}$-loss from $2 \mathrm{H}$ atoms from different rings (Paris et al. 2014). Thus, we will assume that the $\mathrm{H}_{2}$-loss comes from $2 \mathrm{H}$ atoms in a duo ring. In the case of coronene derivatives, this indicates that direct $\mathrm{H}_{2}$-loss will be considered only for the molecules with an even number of $\mathrm{H}$-atoms. Dehydrogenated coronene derivatives with an odd number of $\mathrm{H}$-atoms will only lose the (more loosely bound) $\mathrm{H}$ atom left in the ring. 

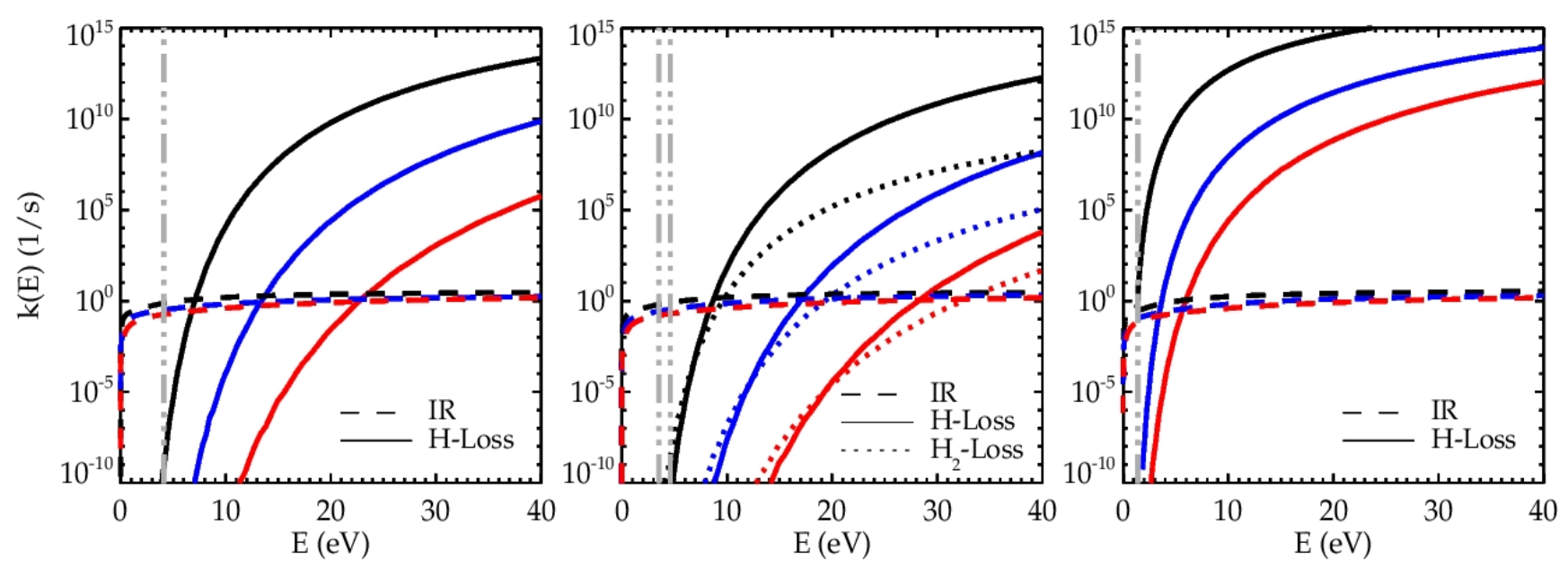

Fig. 5. Comparison of IR emission and photodissociation rates as a function of internal energy of the PAH. The rates determined for coronene derivatives are shown in black, while the rates for circumcoronene and circumcircumcoronene are shown in blue and red respectively, following the color scheme of Fig. 4. The grey vertical lines show the activation energies assumed listed in Table 2. The left panel shows the rates for the first dehydrogenated state of each parent molecule in their neutral state $\left(\mathrm{C}_{24} \mathrm{H}_{11}^{0}, \mathrm{C}_{54} \mathrm{H}_{17}^{0}\right.$ and $\left.\mathrm{C}_{96} \mathrm{H}_{23}^{0}\right)$, in order to exemplify the rates for molecules with an odd number of $\mathrm{H}$ atoms. The middle panel shows the rates for the parent neutral molecules, illustrating the $\mathrm{H}$ and $\mathrm{H}_{2}$-loss rates for PAHs with an even number of $\mathrm{H}$ atoms. The right panel shows the rates for the first superhydrogenated state of the neutral molecules $\left(\mathrm{i} . e ., \mathrm{C}_{24} \mathrm{H}_{13}^{0}, \mathrm{C}_{54} \mathrm{H}_{19}^{0}\right.$, and $\mathrm{C}_{96} \mathrm{H}_{25}^{0}$ ) for which no direct $\mathrm{H}_{2}$-loss is considered. IR emission rates as a function of internal energy, $k_{I R}(\mathrm{E})$, have been calculated using the IR cross sections of each molecule whenever available (see Appendix A). For $\mathrm{C}_{24} \mathrm{H}_{11}^{0}$ and $\mathrm{C}_{96} \mathrm{H}_{23}^{0}$ we have assumed the intrinsic frequencies of the parent molecules.

In the case of $\mathrm{C}_{54} \mathrm{H}_{18}$ and $\mathrm{C}_{96} \mathrm{H}_{24}$ derivatives (which have solo and duo rings), the $\mathrm{H}_{2}$ molecule will also be assumed to form from $2 \mathrm{H}$ atoms from the same ring (a duo ring). In other words, direct $\mathrm{H}_{2}$-loss will only be considered for molecules having an even number of $\mathrm{H}$ atoms until only solo $\mathrm{H}$ atoms remain in the molecule. These solo $\mathrm{H}$ atoms will be lost 1 by 1 using the activation energies and entropies discussed before (see Table 2).

Dissociation rates. In order to obtain the multiphoton dissociation rates, we first calculate each rate constant according to the Rice-Rampsberger-Kassel-Marcus (RRKM) theory in the Arrhenius form, using the parameters described above (Tielens 2005).

IR emission rates, $k_{\mathrm{IR}}\left(Z, N_{\mathrm{H}}, E\right)$, were calculated using the so-called thermal approximation (e.g., Schutte et al. 1993; Bakes et al. 2001). In this approximation, the emission from a given vibrational mode corresponds to the average emission of an oscillator connected to a thermal bath at temperature $T$. The inverse timescale of IR emission is then given by:

$k_{I R}\left(Z, N_{\mathrm{H}}, E\right)=4 \pi \sum_{i} \sigma_{\mathrm{IR}_{i}} \frac{B\left(\bar{v}_{i}, T(E)\right)}{E}$,

where $B\left(\bar{v}_{i}, T(E)\right)$ is the Planck function, and $\sigma_{\mathrm{IR}_{i}}$ is the IR cross section of mode $i$ at frequency $\bar{v}_{i}$. The $T(E)$ relation is derived from the heat capacity $C_{v}(T)$ calculated for each molecule (Bakes et al. 2001). Figure 5 compares the calculated IR emission and dissociation rates. $\mathrm{H}_{2}$-loss dominates over $\mathrm{H}$-loss only at low internal energies, where IR emission dominates over both dissociation processes. At an internal energy of $9 \mathrm{eV}, \mathrm{H}-$ loss becomes faster than IR emission for neutral coronene. This transition occurs at $17 \mathrm{eV}$ and $\sim 28 \mathrm{eV}$ for $\mathrm{C}_{54} \mathrm{H}_{18}^{0}$ and $\mathrm{C}_{96} \mathrm{H}_{24}^{0}$ respectively, way above the Lyman limit. The same behaviour is observed for the other ionization states, where the crossing point between the H-loss rate and IR relaxation occurs at slightly higher energies (up to $2 \mathrm{eV}$ ) due to the faster IR emission rates of ions compared to those of neutrals. For dehydrogenated states with an odd number of $\mathrm{H}$-atoms, for which we have assumed a smaller activation energy, the crossing point occurs at energies $3-5 \mathrm{eV}$ smaller than for the molecules with an even number of $\mathrm{H}$-atoms, but this is still above the Lyman limit for the larger molecules. For superhydrogenated states, on the other hand, the transition occurs at much lower energies, all below $13.6 \mathrm{eV}$. Comparing our estimates with Montillaud et al. (2013) for the parent molecules, we get that the H-loss rates as a function of internal energy of the PAH are fairly similar: the curves of Fig. 5 are slightly steeper, leading to smaller rates than theirs at lower energies (near the crossing points), but similar at higher energies. Clearly for superhydrogenated molecules, our rates are significantly larger than theirs given that we assume low energies compared to their $3.2 \mathrm{eV}$ dissociation energy for $\mathrm{H}$-loss from $N_{\mathrm{H}}^{0}+1$ molecule (their maximum hydrogenation state). Montillaud et al. (2013) also compares their rates to the values used in Berné \& Tielens (2012) and Le Page et al. (2001). Somewhat different rates are obtained due to the different adopted binding energies (3.3 eV for the H-loss in Berné \& Tielens 2012; and $4.8 \mathrm{eV}$ in Le Page et al. 2001) and pre-exponential factors $\left(3 \times 10^{16} \mathrm{~s}^{-1}\right.$ in both studies). For coronene, the H-loss rates of Montillaud et al. (2013) are higher than the ones in Le Page et al. (2001). Compared to Berné \& Tielens (2012), the crossing points occur at slightly different energies, but due to the steeper change of the rates of Montillaud et al. (2013), their rates are higher at energies above $11 \mathrm{eV}$.

Assuming that the cooling of a PAH can be described as a Poisson process, we can estimate the temperature probability function of the PAH in a given radiation field following Bakes et al. (2001; see also Purcell 1976; Aannestad \& Kenyon 1979). The temperature probability function for a PAH exposed to photons of energy $E, G(T, E)$, is then given by:

$G(T, E) \mathrm{d} E=\frac{\bar{r}}{\mathrm{~d} T / \mathrm{d} t} \exp \left(-\bar{r} \tau_{\min }(T, E)\right) \mathrm{d} E$,

with $\bar{r}$ the photon absorption rate, $\mathrm{d} T / \mathrm{d} t$ the cooling rate, and $\tau_{\min }$ the minimum amount of time the PAH requires to cool from 

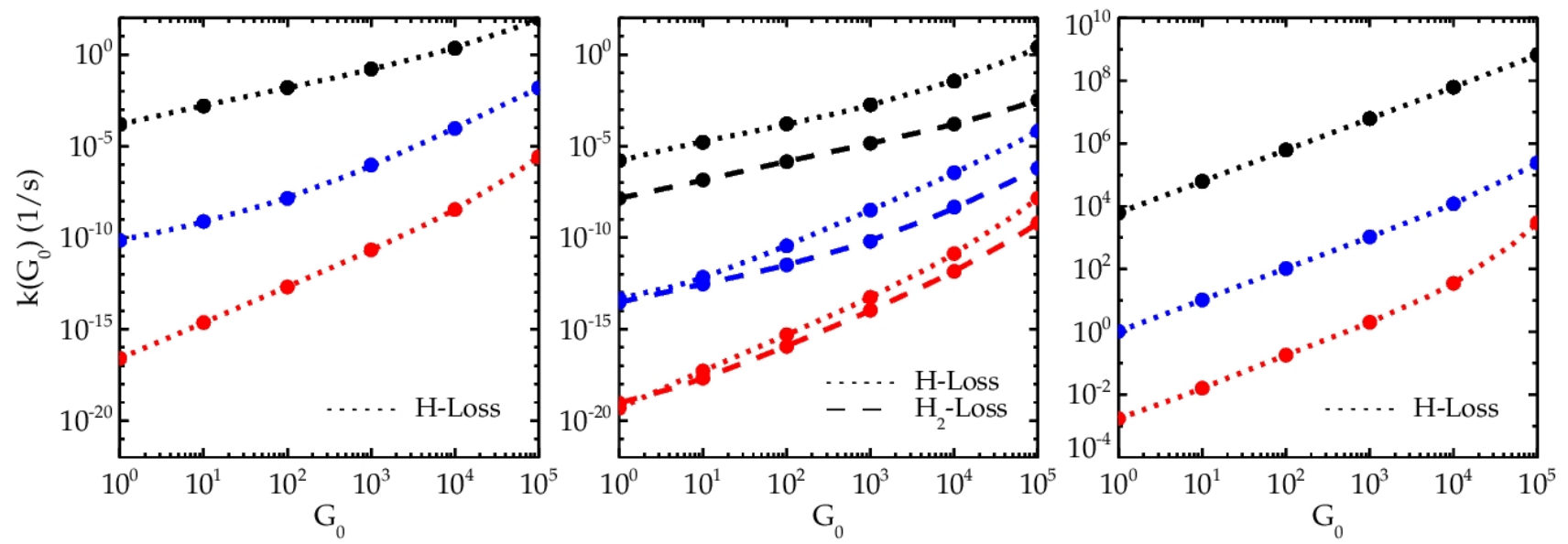

Fig. 6. Variation of the photodissociation rates with respect to the intensity of the UV field, $G_{0}$. The rates are shown for the same molecules as in Fig. 5, following the same color scheme. For the smallest molecule (black curves) the rates scale linearly with $G_{0}$ at the range of interest for NGC $7023\left(G_{0} \leq 2600\right)$ indicating single photon events occurrence for coronene derivatives. For the larger molecules, multiphoton events are important. Even though $\mathrm{H}$-loss is the dominant dissociation channel, at low $G_{0}$ values, $\mathrm{H}_{2}$-loss can become a competitive process for the larger circumcoronene and circumcircumcoronene species.

a maximum temperature $T_{\max }(E)$ to $T$, after absorbing a photon of energy $E$. Since the PAH is absorbing photons in a stellar radiation field $n(E)$, expression (4) must be averaged over the distribution of photon energies:

$\tilde{G}(T)=\frac{1}{\bar{r}} \int G(T, E) n(E) \mathrm{d} E$,

so that $\tilde{G}(T) \mathrm{d} T$ corresponds to the probability of finding the PAH at a temperature between $T$ and $T+\mathrm{d} T$ given a single photon event. When considering multiphoton events, we must calculate the temperature probability function for $n$ photon events, $G_{n}(T)$, in an iterative fashion:

$G_{n}(T)=\int G_{n-1}\left(T_{i}, T^{\prime}\right) G\left(T^{\prime}, T\right) \mathrm{d} T^{\prime}$,

where we are essentially calculating the probability for a $\mathrm{PAH}$ to be at temperature $T$ given that $n-1$ photon events take the PAH to a temperature $T^{\prime}$ starting from an initial temperature $T_{i}$, and an additional photon event takes the PAH from $T^{\prime}$ to a temperature $T$.

We start by calculating $G_{1}(T)$ considering an initial temperature $T_{i}$. In order to make sure the probability distribution is well defined (i.e., integral over $T$ gives unity), we choose a proper initial temperature $T_{i}$ and a temperature grid for each molecule, based on where the peak of the distribution is expected to be, i.e., the temperature $T_{\text {peak }}$ at which $\mathrm{d} T / \mathrm{d} t=\bar{r}$. This procedure also helps in decreasing the time it takes for the calculations to run. At each step we check that $T_{i}>0 \mathrm{~K}$ and that the integral $\int G_{1}(T) \mathrm{d} T \sim 1$ to within a few percent. Once $G_{1}(T)$ is obtained following Bakes et al. (2001), we estimate the H-loss, $\mathrm{H}_{2}$-loss and IR emission rates for each PAH $\left(Z, N_{\mathrm{H}}\right)$ in a given hydrogenation and ionization state as:

$k_{i}=\int k_{i}(T) G_{n}(T) \mathrm{d} T$.

Once convergence is reached between successive iterations, we calculate the probability for each reaction $i$ as:

$P_{i}=\frac{k_{i}}{k_{\mathrm{tot}}}$ where: $k_{\mathrm{tot}}=k_{\mathrm{H}, \text { Loss }}+k_{\mathrm{H}_{2} \text {,Loss }}+k_{\mathrm{IR}}$. We perform this calculation for each PAH $\left(Z, N_{\mathrm{H}}\right)$ for different intensities of the radiation field, i.e., varying $G_{0}$ between 1 and $10^{6}$. Figure 6 shows $k_{\mathrm{H}, \mathrm{Loss}}\left(G_{0}\right)$ and $k_{\mathrm{H}_{2}, \text { Loss }}\left(G_{0}\right)$ as a function of $G_{0}$ for the molecules presented in Fig. 5. Fits to some of these curves can be found in the Appendix B. The variation of both rates scales linearly with $G_{0}$ for coronene up to very high $G_{0}$ values, meaning this PAH dissociates through single photon events in our range of interest. Larger molecules need more photons to dissociate (Montillaud et al. 2013): $\mathrm{H}$-loss for $\mathrm{C}_{54} \mathrm{H}_{18}^{0}$ is already a 2 photons event at $G_{0}<10$, while at the edge of the NGC 7023 NW PDR, it is expected to be a 3 photons event. $\mathrm{C}_{96} \mathrm{H}_{24}^{0}$ on the other hand, needs $\geq 3$ photons depending on the $G_{0}$ considered (at $G_{0}>10^{5}$ it becomes a 4 photons event). As $\mathrm{H}_{2}$-loss occurs at low internal energies of the PAH, it scales linearly with UV field intensity in highly shielded environments, becoming a competitive process with respect to the direct $\mathrm{H}$-loss for the larger molecules $\left(N_{\mathrm{C}}>54\right)$.

The final rate of dissociation for each process is given by the rate of photon absorptions times the probability for each channel to occur given by expression (8).

Electronic relaxation. As an aside, we note that for small neutral species, electronic fluorescence and phosphorescence can play a role as a de-excitation process. However, for coronene, mean fluorescence and phosphorescence yields are of only 0.2 and 0.1, respectively (Dawson \& Kropp 1969). Because of smaller energy gaps, fluorescence and phosphorescence for large species is unimportant (Pino et al. 2011). For ions, internal conversion will be even more important as the energy gaps involved are smaller (cf. Pino et al. 2011). Delayed fluorescence - also sometimes called Poincaré fluorescence, where the excited species revisits the $S_{1}$ (or $T_{1}$ ) state (Léger et al. 1988) - is measured to be unimportant for coronene as it only accounts for a small fraction of the total radiationless de-activation of triplet coronene (Kropp \& Dawson 1967). While delayed fluorescence cannot compete with IR relaxation for low levels of excitation, at high internal energies it does become more important. As fragmentation, particularly for the large species, requires high levels of internal energy, delayed fluorescence may play more of 
a role. Following Berné et al. (2015), and adopting a binding energy of $4.6 \mathrm{eV}$ and an energy gap of $2.1 \mathrm{eV}$ (Fusaro 2012), we have examined the possible effect of electronic relaxation on the H-loss rate of large PAHs. As an example, for circumcoronene, at an internal energy of $25 \mathrm{eV}$ the $\mathrm{H}$-loss rate is calculated to be $5 \times 10^{4} \mathrm{~s}^{-1}$, which should be compared to the electronic relaxation rate of $\sim 0.5 \mathrm{~s}^{-1}$ and the IR relaxation rate of $\sim 1$ $\mathrm{s}^{-1}$. Likewise for circumcircumcoronene at an internal energy of $37 \mathrm{eV}$, the $\mathrm{H}$-loss rate is $10^{3} \mathrm{~s}^{-1}$, the electronic relaxation rate is $\sim 1 \mathrm{~s}^{-1}$ and the IR rate is $\sim 1 \mathrm{~s}^{-1}$. Hence we have chosen to ignore electronic fluorescence.

\subsubsection{Reactivity with hydrogen}

For cationic species we will consider the works of Le Page et al. (1997) and Betts et al. (2006). These studies have experimentally determined rate coefficients for the first and second hydrogenation of small cationic PAHs $\left(N_{\mathrm{C}} \leq 24\right)$. The rates for all PAHs agreed within the uncertainties with the rate derived for coronene, $1.4 \pm 0.7 \times 10^{-10} \mathrm{~cm}^{3} / \mathrm{s}$.

More recent experiments on the hydrogenation of coronene cation reveal that only coronene cations with an odd number of H-atoms are detected through mass spectrometry (Boschman et al. 2012; Reitsma et al. 2014). Transition states calculations predict a small $(0.01 \mathrm{eV})$ barrier for the first hydrogenation to a duo ring, and a barrier of $0.03 \mathrm{eV}$ for the second one within the same ring (Cazaux et al. 2016). Further H-additions have different barriers depending on both, the radical nature of the reactants and the deformation in the carbon structure of the PAH. Low barriers are typically of the order of $0.01 \mathrm{eV}$, while large barriers are typically of the order of $0.03 \mathrm{eV}$. As a reference, a $0.01 \mathrm{eV}$ barrier leads to a rate similar as the one derived by Le Page and coworkers assuming the geometrical cross section of coronene $\left(35.7 \AA^{2}\right)$. Given the lack of data on the hydrogenation of dehydrogenated states, we will adopt the rate of $1.4 \times 10^{-10} \mathrm{~cm}^{3} / \mathrm{s}$ for all hydrogenation steps of all positively ionized species, irrespective of the barriers.

For neutral species we will consider the work of Rauls \& Hornekær (2008) on the hydrogenation of neutral coronene. This work predicts a barrier of $0.06 \mathrm{eV}$ for the first hydrogenation; no barrier for the second; a 0.03 or $0.04 \mathrm{eV}$ barrier for the third extra $\mathrm{H}$ atom depending on whether the $\mathrm{H}$ atom is attached to an inner or outer edge of the molecule; and no barriers for additional hydrogenations up to 8 extra $\mathrm{H}$ atoms. Considering the geometrical cross section of coronene, a $0.06 \mathrm{eV}$ barrier leads to rates of the order of $10^{-10} \mathrm{~cm}^{3} / \mathrm{s}$ at the temperature range of relevance (few hundred K). Given the lack of data on the larger molecules we decide to use the geometrical cross sections of each molecule together with the barriers given in Rauls \& Hornekær (2008) for all neutral species in normally-to-superhydrogenated states. For the dehydrogenated molecules we do not consider any barrier, and we adopt a $7 \%$ efficiency as found for cations by Demarais et al. (2014).

Regarding anionic species, Demarais et al. (2012) performed experiments on the reactivity of deprotonated small PAH anions with $\mathrm{H}$ atoms, and found that hydrogenation occurs through associative detachment (i.e., formation of the neutral parent molecule and the release of an electron). For the largest PAH they studied, $\mathrm{C}_{14} \mathrm{H}_{9}^{-}$, they found a reaction rate of $(7.8 \pm$ $0.2) \times 10^{-10} \mathrm{~cm}^{3} / \mathrm{s}$ and report that, although energetically feasible, the pure association reaction $\left(\mathrm{C}_{14} \mathrm{H}_{9}^{-}+\mathrm{H} \rightarrow \mathrm{C}_{14} \mathrm{H}_{10}^{-}\right)$is not observed. Thus, we will consider the associative detachment pathway as the $\mathrm{H}$-addition process for all negatively ionized species considered in our work $(Z<0)$. In their study, the reaction rate decreases slightly with increasing size of the PAH. However given their small sample (benzene, naphthalene and anthracene), and the lack of data on larger PAHs, we will adopt the rate determined for anthracene for all our anionic molecules.

It is important to mention that no $\mathrm{H}_{2}$-addition reactions will be considered for any of the molecules since the few experiments that have been carried out on small cationic PAHs have detected little, if not any, reactivity with $\mathrm{H}_{2}$, at least not above a detection threshold of $5 \times 10^{-13} \mathrm{~cm}^{3} / \mathrm{s}$ (Le Page et al. 1997; Demarais et al. 2012).

\subsection{5. $\mathrm{H}_{2}$ abstraction}

$\mathrm{H}_{2}$ abstraction from superhydrogenated neutral coronene has been observed in desorption experiments on coronene bombarded with deuterium atoms (Thrower et al. 2011). This has also been analyzed from a theoretical perspective in the work of Rauls \& Hornekær (2008). They predict that the $\mathrm{H}_{2}$ abstraction (occurring at the outer edge) from $\mathrm{C}_{24} \mathrm{H}_{13}^{0}$ is a barrier-less process, while the abstraction from $\mathrm{C}_{24} \mathrm{H}_{14}^{0}$ has a small barrier of $0.01 \mathrm{eV}$. Further abstractions occur in barrier-less processes. Bauschlicher \& Bakes (2001) also predict no barriers for $\mathrm{H}_{2}$ abstraction from $\mathrm{C}_{24} \mathrm{H}_{13}^{-}$and $\mathrm{C}_{54} \mathrm{H}_{19}^{-}$. Experiments on deuteration of coronene films taken at $300 \mathrm{~K}$ suggest abstraction cross sections of $0.06 \AA^{2}$ (Mennella et al. 2012). Considering this cross section for all abstractions, $\sigma_{\mathrm{er}}$, then the Eley-Rideal rate is given by:

$k_{\mathrm{er}}=\sigma_{\mathrm{er}}\left(\frac{8 k_{\mathrm{B}} T_{\mathrm{gas}}}{\pi m_{\mathrm{H}}}\right)^{1 / 2} n(\mathrm{H})$,

which translates to a rate:

$k_{\mathrm{er}}=8.7 \times 10^{-13} \sqrt{\frac{T_{\mathrm{gas}}}{100}} n(\mathrm{H})$.

\section{Model}

Once the physical conditions and all processes are defined for each $\mathrm{PAH}$, we proceed to establish the kinetic equation for the abundance of each PAH considered in the model (see Appendix C). We solve the system of equations assuming steady state i.e., $\mathrm{d}\left[\mathrm{PAH}\left(Z, N_{\mathrm{H}}\right)\right] / \mathrm{d} t=0$. Once the distribution of $\mathrm{PAH}$ abundances were obtained for each species, we calculate the emission spectra following Bakes et al. (2001), i.e., using the temperature probability function described in the previous section. In order to analyze the emission band ratios, we consider an intrinsic shift of the bands of $15 \mathrm{~cm}^{-1}$, and Gaussian line profiles with a FWHM of $15 \mathrm{~cm}^{-1}$ for all spectra (see Boersma et al. 2013). The results regarding the spectral variations will be presented in Sect. 4.3.

\section{Results}

Here we present the results of our work in terms of the distribution of abundances (Sect. 4.1), the $\mathrm{H}_{2}$ formation rates (Sect. 4.2), and the spectral variations expected from the derived distribution of PAH species (Sect. 4.3).

\subsection{Abundances}

In agreement with Montillaud et al. (2013) and Boschman et al. (2015), the hydrogenation of PAHs varies strongly with PAH 
H. Andrews et al.: Hydrogenation and dehydrogenation of interstellar PAHs: Spectral characteristics and $\mathrm{H}_{2}$ formation
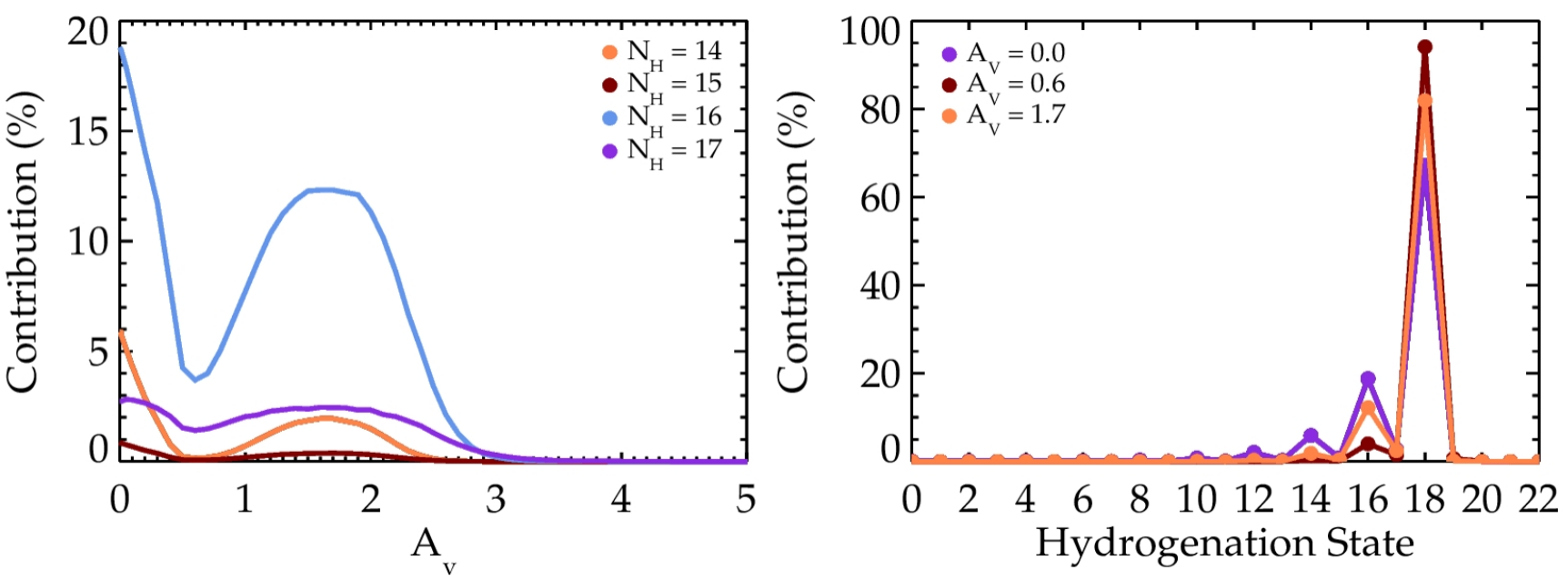

Fig. 7. Left panel: relative contribution of dehydrogenated states of circumcoronene as a function of depth into the cloud, $A_{V}$. The contributions consider all ionization states at which the molecules are present. The curves are shown for the first 4 dehydrogenated states of the parent molecule $\left(N_{\mathrm{H}}=17-4\right)$. Other partially dehydrogenated states contribute in smaller fractions. Right panel: distribution of circumcoronene derivatives at different $A_{V}$ values where partially dehydrogenated species are observed.
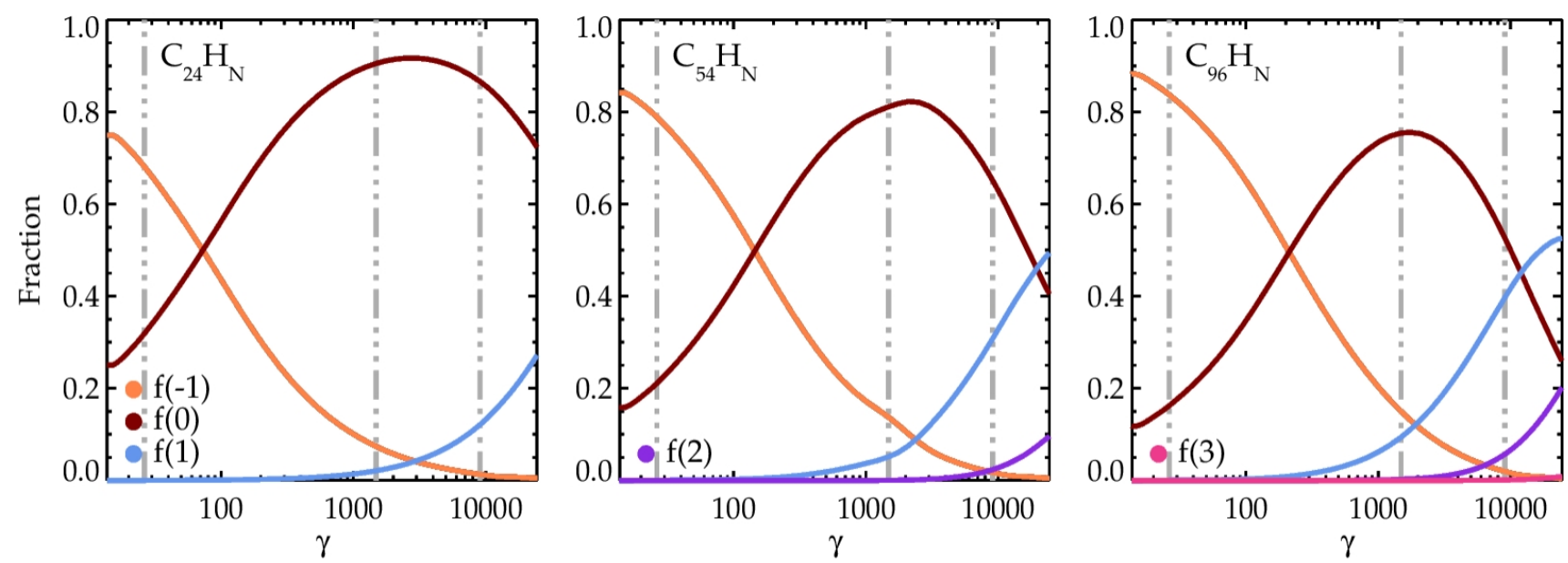

Fig. 8. Charge distribution of the PAHs considered in our study. The contribution from each ionization state is shown as a function of the ionization parameter $\gamma \equiv G_{0} \sqrt{T_{\text {gas }}} / n(e)$ for coronene (left panel), circumcoronene (middle panel) and circumcircumcoronene (right panel) derivatives. The color scheme is the same for all panels. Dashed grey lines are at $A_{V}=1,2$ and 4 . As observed from the panels, the main charge states throughout the cloud are the neutrals $(Z=0)$, anions $(Z=-1)$ and cations $(Z=1)$. Higher positively charged states are only present in minor levels at the surface of the NW PDR of NGC 7023. Higher anionic states $(Z<-1)$ are not observed.

size and depth into the PDR (see Appendix D). While coronene is mostly dehydrogenated throughout most of the cloud - only at an $A_{V} \sim 4.2$ do the first hydrogenated states appear -, circumcircumcoronene does not show any dehydrogenation under the same physical conditions. The medium-sized PAH, circumcoronene, shows an intermediate behaviour compared to the other 2 species, with small traces $(\lesssim 20 \%)$ of singly- and didehydrogenated states at the surface. The dehydrogenated fraction first decreases with depth in the PDR and then increases again slightly around an $A_{V}$ of 1.7 (see Fig. 7).

We also note that both circumcoronene and circumcircumcoronene, show small amounts of superhydrogenated species deep in the cloud $\left(A_{V}>4\right)$ due to the increase in the atomic $\mathrm{H}$ density resulting from UV photons penetrating from the backside of the cloud (this also leads to the appearance of the first hydrogenated states of coronene; see Appendix D). In contrast, Montillaud et al. (2013) reported that circumcircumcoronene is superhydrogenated $\left(N_{\mathrm{H}}^{0}+1\right)$ throughout the entire cloud. The difference mainly lies in the dissociation parameters we have assumed for the superhydrogenated species (Bauschlicher \& Ricca 2014). The low activation energies we have adopted make UV driven H-loss the main dehydrogenation process for superhydrogenated circumcircumcoronene derivatives (e.g., rates of $\sim 10^{-3} \mathrm{~s}^{-1}$ at $A_{V}=0$ ). Test runs of $\pm 0.4 \mathrm{eV}$ in the activation energies presented in Table 2 for the superhydrogenated species, do not have an impact on this aspect.

Regarding ionization, we see that the larger the molecule, the higher the contribution from positively ionized species at the surface of the cloud. In the case of coronene derivatives, we find that neutrals contribute $\sim 73 \%$ to the total abundance of PAHs at an $A_{V}=0$, while cations contribute $\sim 27 \%$. The radiation field intensity is not strong enough to produce dications for this species. For the larger molecules in our sample, the neutral-tocation ratio inverts with respect to coronene. Positively charged circumcoronene derivatives ( $Z=1$ and 2 ) contribute $59 \%$, while neutrals contribute $41 \%$. In the case of circumcircumcoronene, we see higher ionization states than for the smaller species. Here the percentage considering cations and dications adds up to 
$\sim 74 \%$, while it is $26 \%$ for the neutrals. Higher positively ionized species, $Z>2$, contribute at negligible levels $(<1 \%)$. Figure 8 shows the charge distribution variation with respect to the ionization parameter $\gamma \equiv G_{0} \sqrt{T_{\text {gas }}} / n(e)$ for the 3 species considered in our work. As the UV field intensity decreases, neutrals and anions become the dominant charge states. This appearance of anions occurs at lower $A_{V}$ values as the size of the parent molecule increases: a $10 \%$ contribution from anions is expected at a $\gamma=2.2 \times 10^{3}, 2 \times 10^{3}$ and $10^{3}$ for $\mathrm{C}_{96} \mathrm{H}_{24}, \mathrm{C}_{54} \mathrm{H}_{18}$, and $\mathrm{C}_{24} \mathrm{H}_{12}$ derivatives. Indeed, anions become the dominant charge states at large depths in the cloud. At $A_{V}=5$, anions make up $74 \%$, $84 \%$ and $88 \%$ of the coronene, circumcoronene and circumcircumcoronene derivatives population. In contrast, the PAH anion abundance is only of $1-2 \%$ at the $\mathrm{HI}$ to $\mathrm{H}_{2}$ transition $\left(A_{V} \sim 1\right)$ depending on the molecule. However, negatively charged species are already important at an $A_{V} \sim 2$, especially for the larger species.

While test runs show that the use of different ionization yields does not have an impact on the derived abundances, the adopted dependence of the IPs and EAs on hydrogenation does have an effect on our results, but only for the smallest species in our sample. It is important to recall that previous models on PAH abundances in PDRs have not considered negatively charged species, and/or adopt the IP and EA of the parent molecule for all hydrogenation states within a given charge state (Le Page et al. 2001, 2003; Montillaud et al. 2013; Boschman et al. 2015). Here we find that it is important to use the specific IPs and EAs for each molecule, especially for the smallest species. The large fraction of negatively charged coronene derivatives results from the adopted EAs of the dehydrogenated states of the molecule, where the EA $>1 \mathrm{eV}$ for $N_{\mathrm{H}}<8$ molecules (see Fig. 4). Indeed if were to adopt the IP and EA of the parent molecule coronene for all de-and-superhydrogenated derivatives, we would obtain that coronene stays neutral in the deepest regions of the cloud (e.g., the electron sticking coefficient is $s_{\mathrm{e}} \sim 10^{-5}$ for an EA $=$ $0.47 \mathrm{eV})$. The larger molecules on the other hand, are not affected by this, since the EA of the parent molecule is large enough to lead to electron attachment.

We have explored the distribution of hydrogenation states of our three species in a systematic manner over the astrophysical relevant $G_{0}-n(\mathrm{H})$ parameter space (Fig. 9). For these calculations we adopted a gas temperature of $500 \mathrm{~K}$. The results show that coronene is mostly completely dehydrogenated. At a given density, for UV fields exceeding $G_{0} \gtrsim 0.01 n(\mathrm{H})$, coronene quickly loses hydrogen and becomes fully dehydrogenated. We emphasize that at that point, carbon loss can become important - limiting the lifetime of the pure carbon cluster - but we have not taken that into account. For $G_{0}$ values less than $\sim 0.05 n(\mathrm{H})$, we can find superhydrogenated coronene molecules contributing at a $10-15 \%$ level. However the greatest contribution in these highly shielded environment comes from partially dehydrogenated species, with very little contribution from the normally hydrogenated molecule $\mathrm{C}_{24} \mathrm{H}_{12}(<10 \%)$.

Unlike coronene, the larger species are far more difficult to dehydrogenate: for circumcoronene, full dehydrogenation requires $G_{0}$ in excess of $\sim 300 n(\mathrm{H})$ at $n(\mathrm{H})=1 \mathrm{~cm}^{-3}$, and above $5 n(\mathrm{H})$ at $n(\mathrm{H})=10^{3} \mathrm{~cm}^{-3}$. On the other hand, our largest species circumcircumcoronene only dehydrogenates for the highest UV fields and lowest densities $\left(G_{0}>5 \times 10^{4}\right.$ and $\left.n(\mathrm{H})<3 \mathrm{~cm}^{-3}\right)$. Normally hydrogenated species dominate below these limits until superhydrogenation sets in at $G_{0} \sim 0.005 n(\mathrm{H})$. For smaller $G_{0}$ values, these large PAHs are superhydrogenated. We note that these transitions from fully dehydrogenated to normally hydrogenated, and from normally hydrogenated to superhydrogenated are very sharp (cf., Tielens 2005). From this we take that any PAH is very sensitive to small variations in the hydrogenation parameter over a particular range, specific to that molecule, and for circumcoronene that partly overlaps with the $G_{0} / n(\mathrm{H})$ range in the NW PDR of NGC 7023 (e.g., the abundance of partially dehydrogenated derivatives of $\mathrm{C}_{54} \mathrm{H}_{18}$ reaches a value of $20 \%$ at $A_{V}=1.8$ where $G_{0} / n(\mathrm{H})=0.95$ and $G_{0} \sim 660 ;$ at $A_{V}=2.2, G_{0} / n(\mathrm{H})=1.35$ and $G_{0} \sim 410$, and their contribution decreases to $\left.\sim 10 \%\right)$. Inspection of the distribution of $\mathrm{C}_{54} \mathrm{H}_{18}$ in Fig. 9 shows that slightly higher $G_{0} / n(\mathrm{H})$ ratios in this $G_{0}-n(\mathrm{H})$ range would have led to complete dehydrogenation of circumcoronene. Given the non-linearity of the mulitphoton dissociation processes for $\mathrm{C}_{54} \mathrm{H}_{18}$, the transition from the normally hydrogenated molecule to the carbon cluster $\mathrm{C}_{54}$ does not occur at a constant $G_{0} / n(\mathrm{H})$ ratio for different $G_{0^{-}}$ $n(\mathrm{H})$ values, and thus we do not see more dehydrogenation at $A_{V}=2.4$, where $G_{0} / n(\mathrm{H}) \sim 1.43$ and $G_{0} \sim 325$.

\section{2. $\mathrm{H}_{2}$ Formation}

We have included 3 possible dehydrogenation pathways for the PAHs in our study: direct loss of an $\mathrm{H}$ atom or an $\mathrm{H}_{2}$ molecule after photon absorption, and $\mathrm{H}_{2}$ abstraction described as an EleyRideal process from superhydrogenated molecules only. Thus, in our model, $\mathrm{H}_{2}$ can be formed from the direct loss of 2 duo $\mathrm{H}$ atoms as an $\mathrm{H}_{2}$ molecule after photon absorption, or from $\mathrm{H}_{2}$ abstraction after superhydrogenation of the normally hydrogenated molecule.

According to our results, direct H-loss is the dominant photodissociation channel for all the molecules in our work. In the case of coronene, complete dehydrogenation is very effective at the surface of the cloud. Direct $\mathrm{H}_{2}$-loss rates at $A_{V}=0$ are two orders of magnitude lower than the direct $\mathrm{H}$-loss rates for this molecule. For the larger PAHs the trend is similar. While direct $\mathrm{H}$-loss is also dominant over $\mathrm{H}_{2}$-loss for circumcoronene and circumcircumcoronene, at some point deep into the cloud $\left(A_{V}>\right.$ $\left.4.5, G_{0}<10\right)$ the two rates become comparable. Inspection of Fig. 6 shows that $\mathrm{H}_{2}$-loss can dominate over $\mathrm{H}$-loss in highly shielded environments where $G_{0}<10$. However in such UVattenuated regions increasing hydrogen densities would tend to superhydrogenate these PAHs, and these, if they were to fragment in any way, would preferentially lose an $\mathrm{H}$ atom and not an $\mathrm{H}_{2}$ molecule.

Regarding $\mathrm{H}_{2}$ abstraction by $\mathrm{H}$ from superhydrogenated PAHs, we find this mechanism not particularly relevant for the conditions in the NW PDR of NGC 7023. Since the formation of $\mathrm{H}_{2}$ through Eley-Rideal abstraction depends on the gas temperature being sufficient to overcome the addition and abstraction barriers, this mechanism is expected to be important at the surface of the PDR, where also the reservoir of atomic hydrogen is large enough to carry out the addition and abstraction processes. For the smallest molecule in our sample, we obtain that coronene can never reach superhydrogenated states at low $A_{V}$ values, and hence cannot form $\mathrm{H}_{2}$ through this mechanism. Boschman et al. (2015) considered $\mathrm{H}_{2}$ abstraction by $\mathrm{H}$ for a wide range of hydrogenation states of coronene $\left(0 \leq N_{\mathrm{H}} \leq 36\right)$, and also found that this is not a dominant process because this PAH is rapidly stripped off of all its hydrogens.

Since large PAHs are expected to be more stable against dehydrogenation, and also more prone towards superhydrogenation than the smaller species, they are expected to be good candidates for the $\mathrm{H}_{2}$ formation through Eley-Rideal $\mathrm{H}_{2}$ abstraction in PDRs. While we do recognize the great stability of large PAHs against dehydrogenation, our results for circumcoronene and 
H. Andrews et al.: Hydrogenation and dehydrogenation of interstellar PAHs: Spectral characteristics and $\mathrm{H}_{2}$ formation
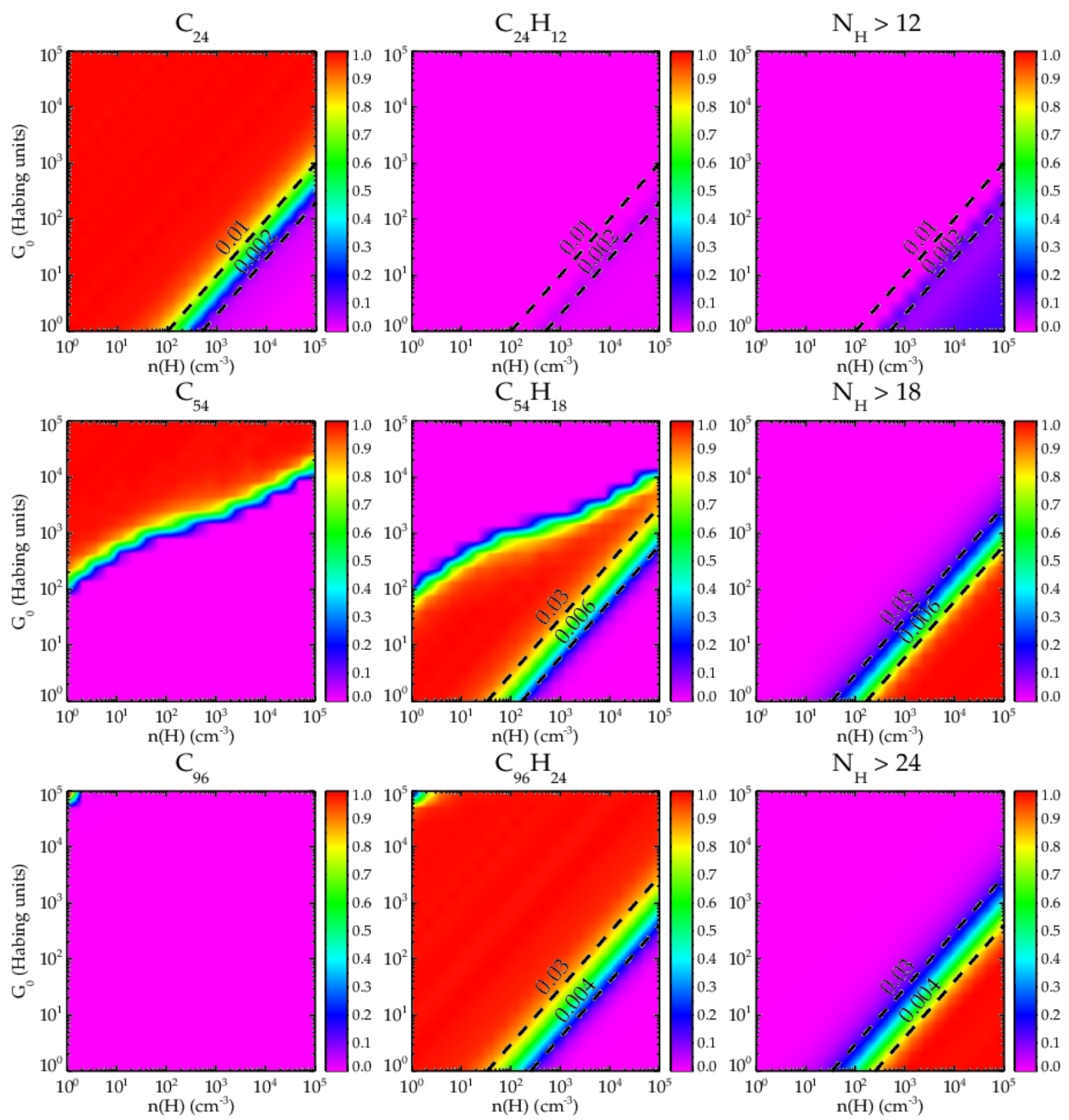

Fig. 9. Fractional contribution from coronene (top panels), circumcoronene (middle panels) and circumcircumcoronene (bottom panels) derivatives for different $G_{0}$ and $n(\mathrm{H})$ values. The contributions include all ionization states. The left column shows the contribution from the completely dehydrogenated molecules, $\mathrm{C}_{24}, \mathrm{C}_{54}$ and $\mathrm{C}_{96}$; the middle column shows the fraction of the normally hydrogenated molecules; and the right column shows the distribution of the superhydrogenated species. In all cases, we have assumed a fraction of molecular hydrogen of 0.5 , and a carbon abundance of $3.5 \times 10^{-4}$ relative to hydrogen. Black lines represent constant $G_{0} / n(\mathrm{H})$ ratios and are given as a reference.

circumcircumcoronene show that by the time these molecules are indeed superhydrogenated, it is already at such high extinction values $\left(A_{V}>4.5\right)$, that the gas temperature is too low to lead to high abstraction rates on PAHs.

We have calculated the efficiencies of $\mathrm{H}_{2}$ formation from PAHs through direct $\mathrm{H}_{2}$-loss (i.e., photodissociation of PAHs) and $\mathrm{H}_{2}$ abstraction by $\mathrm{H}$ (from superhydrogenated species). The efficiencies, $\xi_{H_{2}}$, for a PAH of a given size (i.e., number of $\mathrm{C}$ atoms) and ionization state $Z$, have been defined as the number of $\mathrm{H}$ atoms that collide with the PAH molecules, that are converted to $\mathrm{H}_{2}$ :

$\xi_{\mathrm{H}_{2}}(Z)=\sum_{i} \frac{2 k_{\mathrm{H}_{2}}(Z, i) f(Z, i)}{k_{\mathrm{col}}}$,

where the sum is over all hydrogenation states, and $k_{\mathrm{col}}$ is a fiducial collision rate of $\mathrm{H}$-atoms with the $\mathrm{PAH}$ species, whose expression depends on the PAH charge state under consideration (see Appendix E). In the case of direct $\mathrm{H}_{2}$-loss, $k_{\mathrm{H}_{2}}$ corresponds 

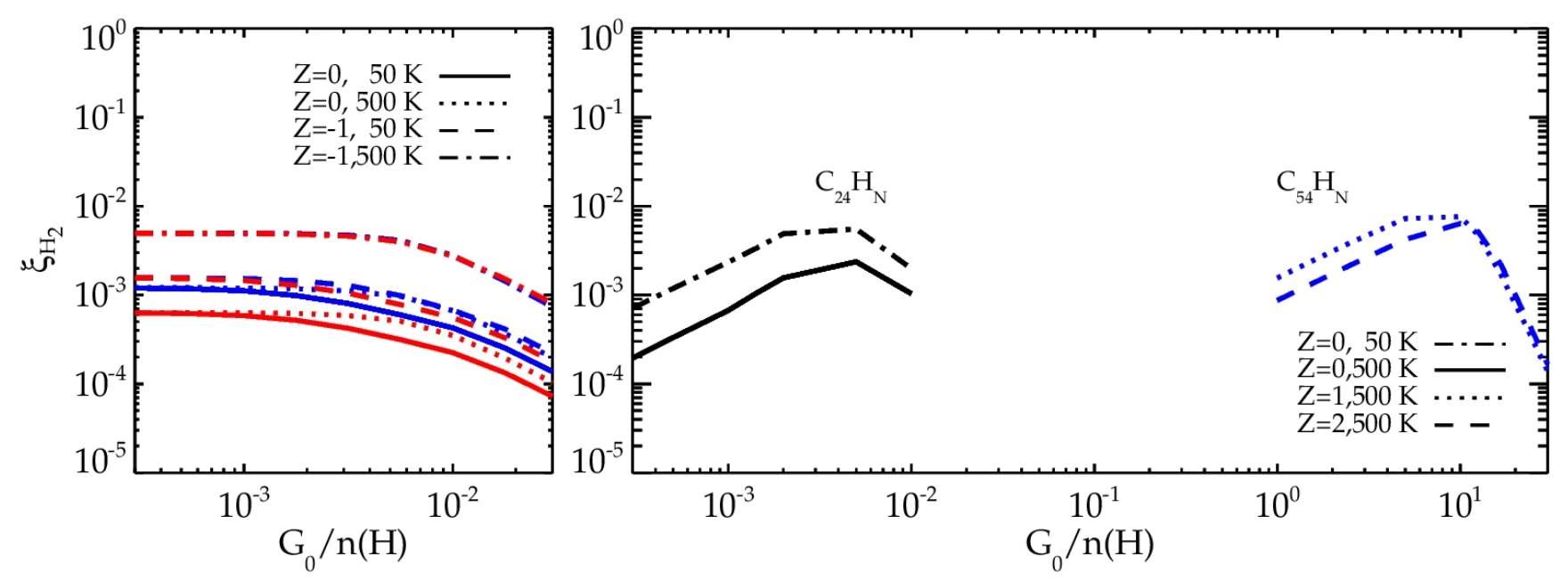

Fig. 10. Efficiencies of $\mathrm{H}_{2}$ formation from PAHs. The left panel shows the efficiencies of $\mathrm{H}_{2}$ formation through Eley-Rideal abstraction from superhydrogenated species as a function of $G_{0} / n(\mathrm{H})$. Estimates for circumcoronene and circumcircumcoronene superhydrogenated species are shown in blue and red, respectively. The efficiencies have been derived for neutrals and anions, since these are the charge states at which superhydrogenated species can be found according to our model. Two gas temperatures have been assumed as a reference. The right panel shows the efficiencies of $\mathrm{H}_{2}$ formation from photodissociation of PAHs. The efficiencies have been derived for neutral coronene at $G_{0}=10$, and $Z>0$ circumcoronene at $G_{0}=10^{3}$. We have considered different $G_{0}$ values due to the different dehydrogenation behaviour of the species in the $G_{0}-n(\mathrm{H})$ parameter space (see Fig. 9). As it can be derived from the figure, efficiencies stay below $1 \%$ for both mechanisms.

to the $\mathrm{H}_{2}$ photodissociation rate of species with $i$ number of $\mathrm{H}$ atoms in a given charge state; and $f(Z, i)$ corresponds to the fraction of PAHs in charge state $Z$ that release $\mathrm{H}_{2}$ through photodissociation, i.e., normal-to-partially dehydrogenated species with an even number of $\mathrm{H}$ atoms greater than the number of solo $\mathrm{H}$ atoms. For its part, in the case of $\mathrm{H}_{2}$ abstraction, $k_{\mathrm{H}_{2}}$ corresponds to the $\mathrm{H}_{2}$ abstraction rates, $k_{\mathrm{er}}$, in units of $1 / \mathrm{s}$ (Eq. (10)); and $f(Z, i)$ corresponds to the fraction of PAHs in the respective charge state that go through $\mathrm{H}_{2}$ abstraction, i.e., superhydrogenated PAHs with $i$ number of $\mathrm{H}$ atoms.

Figure 10 shows the efficiencies for the 3 PAHs in different environments. The left panel shows the efficiencies of $\mathrm{H}_{2}$ abstraction from the anion and neutral circumcoronene and circumcircumcoronene molecules. We have plotted these ionization states, since they are the ionization states of superhydrogenated species, whenever they are abundant. The efficiencies have been calculated for $T_{\text {gas }}=50 \mathrm{~K}$ and $500 \mathrm{~K}$ for $G_{0} / n(\mathrm{H})$ ratios over which superhydrogenated species are present, going from a $\sim 10 \%$ abundance at $G_{0} / n(\mathrm{H})=0.03$ to a $\sim 100 \%$ abundance at $G_{0} / n(\mathrm{H})=10^{-4}$ for both, circumcoronene and circumcircumcoronene species. In this same range, the charge state of the population goes from $Z=0$ and $Z=-1$ states in a 1:3 ratio, to being $Z=-1$ dominated. The resulting efficiencies increase towards lower $G_{0} / n(\mathrm{H})$ ratios, reaching a plateau once the distribution of species in a given charge state is completely dominated by the PAHs with the largest amount of extra $\mathrm{H}$ atoms considered $\left(f\left(Z, N_{\mathrm{H}}^{0}+4\right) \sim 1\right)$. Overall, $\mathrm{H}_{2}$ formation efficiencies of anions are greater than those of neutral molecules. However, efficiencies are below $1 \%$. The values derived for anionic species are rather independent of $\mathrm{PAH}$ size, but depend on temperature given the fiducial collision rate we adopted. On the other hand, the efficiencies derived for neutral PAHs depend on the geometrical cross section of each PAH. At low $G_{0} / n(\mathrm{H})$ ratios, the efficiencies are independent of the gas temperature. For larger $G_{0} / n(\mathrm{H})$ ratios, the apparent mild temperature dependence is due to $<5 \%$ differences in the derived fractions of superhydrogenated species within each charge state. In the particular case of NGC 7023, at $A_{V} \sim 4.8$ the gas temperature is about $50 \mathrm{~K}$ and $G_{0} / n(\mathrm{H}) \sim 0.03$. Superhydrogenated circumcoronene and circumcircumcoronene species contribute at a $10 \%$ level, anions dominate, and the efficiency of $\mathrm{H}_{2}$ abstraction is very low (below $1 \%$ ).

The efficiencies for the direct $\mathrm{H}_{2}$-loss from photodissociation have been plotted for neutral coronene at $G_{0}=10$, and $Z$ $>0$ circumcoronene species at $G_{0}=10^{3}$. These are the relevant charge states for these molecules in the $G_{0} / n(\mathrm{H})$ ratios considered. Neutral coronene derivatives contribute in $70-78 \%$ to the total abundance of coronene species at $G_{0} / n(\mathrm{H})=10^{-4}-10^{-2}$, range over which the contribution from normal-to-partially dehydrogenated species goes from $26 \%$ to $4 \%$. For $G_{0} / n(\mathrm{H})>10^{-2}$ coronene is only found as $\mathrm{C}_{24}$. As for circumcoronene species, $Z=1$ species dominate the distribution at $G_{0} / n(\mathrm{H})=1-10$, and dications start dominating at $G_{0} / n(\mathrm{H})>10$. We have plotted the efficiencies in the $G_{0} / n(\mathrm{H})$ range over which the relevant hydrogenation states for $\mathrm{H}_{2}$-loss are present (i.e., normal-to-partially dehydrogenated states with an even number of $\mathrm{H}$ atoms above $8)$. Their contributions go from $51 \%$ cations and $14 \%$ dications at $G_{0} / n(\mathrm{H})=1$, to $<1 \%$ at $G_{0} / n(\mathrm{H})>25$. At higher $G_{0} / n(\mathrm{H})$ ratios circumcoronene is found as $\mathrm{C}_{54}$ (see Fig. 9). At $G_{0} / n(\mathrm{H})$ $<1$ circumcoronene can be found mainly in its normal hydrogenated neutral state, down to the regime where superhydrogenated species start becoming abundant $\left(G_{0} / n(\mathrm{H})<0.01\right)$. The efficiencies for $\mathrm{H}_{2}$-loss in these environments are below $10^{-4}$. From the Figure we see that efficiencies of direct $\mathrm{H}_{2}$-loss are at a similar level as the efficiencies of $\mathrm{H}_{2}$ abstraction (below $1 \%$ ). Since the dehydrogenation behaviour is highly PAH size dependent (see left and middle columns of Fig. 9), different PAHs can be equally efficient in different environments. This depends on where in the $G_{0}-n(\mathrm{H})$ space, each PAH can be found in the relevant dehydrogenated states for $\mathrm{H}_{2}$-loss. In the case of NGC 7023, circumcoronene is already found in its normal hydrogenated state, while coronene is completely dehydrogenated. PAHs smaller than $N_{\mathrm{C}}=54$ but larger than $N_{\mathrm{C}}=24$ could reach efficiencies of $1 \%$ at the surface of the NW PDR of NGC 7023.

\subsection{Spectral variations}

We calculated the emission spectra of $\mathrm{C}_{24} \mathrm{H}_{12}, \mathrm{C}_{54} \mathrm{H}_{18}$ and $\mathrm{C}_{96} \mathrm{H}_{24}$ derivatives at different points inside the NW PDR of 
NGC 7023 from $A_{V}=0-2$, considering the distribution of species presented in Sect. 4.1 (see Appendix F). The emission spectrum at $A_{V}=0-2$ is essentially determined by the dominant ionization state of the PAHs at each point inside the cloud. This is due to the lack of strong variations in the hydrogenation states of the species in our work. In the case of coronene, this molecule is completely dehydrogenated, and only changes in ionization state, being mostly neutral with cationic species varying their abundance from $27 \%$ at the surface of the cloud, to $2 \%$ at an $A_{V}=2$. Recall that we do not consider destruction of the carbon skeleton which may be important for highly dehydrogenated species. The main features we see in the emission spectra of coronene derivatives are the appearance of a complex of bands at around $5 \mu \mathrm{m}$ (4.95 and $5.12 \mu \mathrm{m})$, related to stretching modes of the triple bonds in dehydrogenated species; the strong band at $6.71 \mu \mathrm{m}$ due to stretching modes of the carbon skeleton; and the bands at $10.13,10.35$ and $20.4 \mu \mathrm{m}$ due to complex deformation modes of the carbon structure. Among these, the bands at 5.12 and $10.35 \mu \mathrm{m}$, although weak, increase in intensity with respect to the $4.95,6.71,10.13$ and $20.4 \mu \mathrm{m}$ bands towards more highly ionized environments.

As stated before, circumcoronene does present levels of dehydrogenation that vary following the change in the $G_{0} / n(\mathrm{H})$ ratio. We do not have the intrinsic spectra for most of the partially dehydrogenated species (see Appendix A). However, between $A_{V}=0$ and 2, partially dehydrogenated species are present only at low $A_{V}\left(A_{V} \sim 0\right)$ where $\sim 34 \%$ of the emitting species are partially dehydrogenated. These species correspond to the first partially dehydrogenated molecules (e.g., $\left.N_{\mathrm{H}}=17-12\right)$, and so we expect their emission to be similar as that of the parent molecule. Inspection of the emission spectra from circumcoronene derivatives reflect then mainly the change in the ionization state of $\mathrm{C}_{54} \mathrm{H}_{18}$. This is apparent from the decrease with $A_{V}$ in the strength of the 6-9 $\mu \mathrm{m}$ stretching modes relative to the 11-15 $\mu \mathrm{m}$ CH out-of-plane bending modes (Langhoff 1996; Allamandola et al. 1999; Peeters et al. 2002). The signature of positively ionized species is also apparent in the broadening of the $11.25 \mu \mathrm{m}$ band, which is in fact due to the appearance of the $11.05 \mu \mathrm{m}$ feature related to $\mathrm{CH}$ out-of-plane bending modes in ionized PAHs (Rosenberg et al. 2011; Boersma et al. 2013).

In the case of circumcircumcoronene, as for circumcoronene, the spectrum is also dominated by the changes in the ionization state of the parent molecule. Hence we see the same signatures of ionization as we see for $\mathrm{C}_{54} \mathrm{H}_{18}$. At the surface of the cloud, circumcircumcoronene molecules are mostly highly positively ionized $(\sim 74 \%)$. Close to where the $\mathrm{HI}$ to $\mathrm{H}_{2}$ transition occurs, the ratio between neutrals and positively ionized species is close to 1.0. At an $A_{V}=1.6$ neutrals still dominate the population with a $73 \%$ abundance, while the contribution from anions has increased to $8 \%$, and positively ionized species contribute $19 \%$ to the total abundance. At an $A_{V}=2$, neutral molecules contribute in $75 \%$, while anions contribute in $15 \%$. We see that this increase in the abundance of anions (and decrease in the abundance of cations) does not have a discernible impact in the emission spectrum.

A comparison between $\mathrm{C}_{54} \mathrm{H}_{18}$ and $\mathrm{C}_{96} \mathrm{H}_{24}$ derivatives spectra shows that the $3.28 / 3.3$ and $3.28 / 11.3$ peak intensity ratios are larger for the intermediate size molecule than for circumcircumcoronene $\left(3.28 / 3.30=3.58\right.$ and $3.28 / 11.3=0.43$ for $\mathrm{C}_{54} \mathrm{H}_{18}$, against 1.92 and 0.14 respectively for $\mathrm{C}_{96} \mathrm{H}_{24}$ ). Both ratios remain relatively constant, which shows that they are not sensitive to the ionization state of the molecules under these conditions. Instead, they do vary with PAH size (Bauschlicher et al. 2008; Ricca et al. 2012; Croiset et al. 2016).
Up to now, we have focused on the behaviour of the PAH bands with depth into the NW PDR of NGC 7023, where physical conditions vary enough to see spectral changes due to ionization but not due to changes in the hydrogenation state of the PAHs. In order to explore the spectral signatures of large variations in the hydrogenation state of our PAHs we have calculated the emission spectra for different $G_{0} / n(\mathrm{H})$ ratios at $G_{0}=10^{3}$. These spectra can be found in the Appendix F. Visual inspection of the spectra of coronene species shows that over much of the parameter space, the spectrum of the fully dehydrogenated molecule dominates, and the spectral changes represent the variation in its ionization state (the abundance of $Z>0$ species varies from $98 \%$ at $G_{0} / n(\mathrm{H})=100$ to $4.5 \%$ at $G_{0} / n(\mathrm{H})=0.1$ ). For $G_{0} / n(\mathrm{H}) \leq 0.01$ partially dehydrogenated derivatives become more relevant. These are mostly anionic and neutral species for which we do not have the IR spectra. The parent molecule is never important (even at a $G_{0} / n(\mathrm{H})=0.001 \mathrm{C}_{24} \mathrm{H}_{12}$ contributes at a $1 \%$ level only), nor the superhydrogenated species (which barely appear at $G_{0} / n(\mathrm{H})=0.001$ contributing at a $5 \%$ level).

The spectra of the larger species show instead the clear footprints of hydrogenation. As seen in Fig. 9, circumcoronene completely dehydrogenates for $G_{0} / n(\mathrm{H}) \gtrsim 100$ at $G_{0}=10^{3}$. The dominant species at $G_{0} / n(\mathrm{H})=100$ is then $\mathrm{C}_{54}^{+2}$ in $83 \%$ for which we do not have the IR spectrum, but whose emission is expected to be similar to that of $\mathrm{C}_{54}^{+}$(see Appendix F), except for an even stronger $5 \mu \mathrm{m}$ band, as typical from carbon cluster cations. Going to lower $G_{0} / n(\mathrm{H})$ values we see circumcoronene mostly in its normally hydrogenated state $\mathrm{C}_{54} \mathrm{H}_{18}$, and thus what we observe are essentially the changes in ionization state, being mostly neutral with an increasing abundance of the anionic molecule (from $7 \%$ to $18.5 \%$ ). This is reflected in the increasing $6.4 / 11.3$ ratio and the red-shift of the bands, intrinsic to anionic species. The contribution from superhydrogenated species in this $G_{0} / n(\mathrm{H})$ range goes from $\sim 3$ to $35 \%$, which is clear from the inspection of the $3 \mu \mathrm{m}$ region.

Regarding our largest species, circumcircumcoronene stays normally hydrogenated throughout most of the $G_{0} / n(\mathrm{H})$ space. Therefore down to a $G_{0} / n(\mathrm{H})=0.1$ we are basically following the variation in the ionization state of $\mathrm{C}_{96} \mathrm{H}_{24}$, from being mostly in $Z=3$ state to neutral. Going from $G_{0} / n(\mathrm{H})=0.1$ to 0.01 the abundance of superhydrogenated species increases to a $19 \%$, which is only clear in the $3 \mu \mathrm{m}$ spectral region, although the signal is rather weak. Only at $G_{0} / n(\mathrm{H}) \leq 0.001$ superhydrogenated $Z=-1$ and $Z=0$ species become the dominant species. Their signal would be noticeable only in the $3 \mu \mathrm{m}$ region, since the spectrum of the parent molecule looks fairly similar to that of the first few superhydrogenated derivatives.

The $3 \mu \mathrm{m}$ region is particularly sensitive to the superhydrogenation level of PAHs (Bernstein et al. 1996; Sandford et al. 2013). The $3.3 \mu \mathrm{m}$ aromatic $\mathrm{CH}$ stretch shows the two components expected for the coronene family: $3.28 \mu \mathrm{m}$ band due to duo hydrogens, and $3.3 \mu \mathrm{m}$ band for the solo hydrogens (Ricca et al. 2012). Even though the differences between the spectrum of the parent molecule and those of superhydrogenated derivatives become far more clear with higher degrees of superhydrogenation, the main differences start to show up in the $3 \mu \mathrm{m}$ region. With superhydrogenation, new bands appear at longer wavelengths. Zooming in into the $3 \mu \mathrm{m}$ region of the intrinsic spectra of superhydrogenated species (see comparative spectra in Appendix F), we can distinguish three main bands at 3.3, 3.41 and $\sim 3.5 \mu \mathrm{m}$ (and weaker bands at 3.57 and $3.67 \mu \mathrm{m}$ ). The $3.5 \mu \mathrm{m}$ band appears already for a mono superhydrogenated PAH. This band is related to the stretching of each $\mathrm{CH}_{2}$ complex. The band at $3.4 \mu \mathrm{m}$ instead requires two additional $\mathrm{H}$ atoms on the ring. This 
band is related to the stretching modes of the pair of $\mathrm{CH}_{2}$ groups within the same (duo) ring. Therefore the $3.4 \mu \mathrm{m}$ band only appears for hydrogenation states $N_{\mathrm{H}}>N_{\mathrm{H}}^{0}+1$. In fact this pattern is not only seen in the theoretical spectra of these PAHs, but also in that of pyrene, $\mathrm{C}_{16} \mathrm{H}_{10}$, and naphthalene, $\mathrm{C}_{10} \mathrm{H}_{8}$, derivatives (for which the spectra are provided by the PAHdb). For this last one, there are experimental (argon matrix) spectra of the first superhydrogenated state of the molecule, where they report bands at 3.24 and $3.51 \mu \mathrm{m}$, but none at $3.4 \mu \mathrm{m}$ (Ricks et al. 2009). Instead both the experimental and theoretical spectrum of $\mathrm{C}_{10} \mathrm{H}_{10}$ (and further superhydrogenated states) do show a $3.4 \mu \mathrm{m}$ band (Bernstein et al. 1996). Thus the appearance of the $3.4 \mu \mathrm{m}$ band appears to be common to PAHs with at least 2 extra $\mathrm{H}$ atoms. Once all $\mathrm{H}$-sites on the PAH become superhydrogenated, the $3.3 \mu \mathrm{m}$ aromatic $\mathrm{CH}$ stretch disappears. We also, stress that the $G_{0} / n(\mathrm{H})$ value at which these superhydrogenated signatures appear depends on the size of the PAH (cf., Fig. 9). Specifically, the superhydrogenated species appear at $G_{0} / n(\mathrm{H})$ $\lesssim 0.01$ for both circumcoronene and circumcircumcoronene, but their spectral signature would be discernible in the $3 \mu \mathrm{m}$ region at $G_{0} / n(\mathrm{H})$ ten times lower, where superhydrogenated species contribute in more than $30 \%$ to the emission.

\section{Discussion}

We found that small PAHs behave quite differently from intermediate and large species. Small PAHs are easily destroyed in most environments; can rarely be found in their normally hydrogenated state; and only for extremely shielded regions (low $G_{0} / n(\mathrm{H})$ ratios) they can be found in superhydrogenated states. The difference in the abundance distribution between intermediate and large PAHs is related to their dehydrogenation behaviour rather than their superhydrogenation behaviour. The larger the $\mathrm{PAH}$, the more likely it can be found in its normal hydrogenated state. Regarding superhydrogenation of PAHs, intermediate and large PAHs can hardly superhydrogenate in the more diffuse parts of PDRs. These species will lose their extra $\mathrm{H}$ atoms very easily, and only in highly shielded environments will reactions with $\mathrm{H}$ atoms keep the molecules superhydrogenated. These molecules when dominating the abundance distribution of a given species will be mostly in $Z=0$ and $Z=-1$ charge states. Therefore their spectral signature - if observable - would not only be straightforward from the appearance of more bands in the $3 \mu \mathrm{m}$ region, but it would also be discernible from a redshift of the bands (intrinsic to anionic species).

In the particular case of the NW PDR of NGC 7023, we find that small PAHs like coronene will remain completely dehydrogenated throughout most of the cloud. Intermediate and large PAH molecules will remain, mainly, normally hydrogenated but highly positively ionized at the surface of the PDR, where the bulk of the PAH emission is observed. Superhydrogenation is only achieved for the larger species in the more shielded regions of the cloud. Recall we have considered a maximum of 4 additional $\mathrm{H}$ atoms as the maximum superhydrogenated state of a PAH. Test runs showed PAHs do not superhydrogenate further in the case of the NW PDR of NGC 7023. Higher superhydrogenation levels can be achieved in more shielded environments; e.g., considering higher hydrogenated states than $N_{\mathrm{H}}^{0}+4$, circumcoronene derivatives with $N_{\mathrm{H}}>22$ start appearing at $G_{0} / n(\mathrm{H})<$ 0.01 at $G_{0}=1000$, increasing their abundance gradually towards more shielded environments, becoming a $50 \%$ of the emitting population at $G_{0} / n(\mathrm{H}) \sim 0.002$. Such abundance of highly superhydrogenated molecules would leave a clear footprint in the emission spectra. However, nor the molecular properties nor the energetics involved in the $\mathrm{H}$-addition/ $\mathrm{H}_{2}$-abstraction processes have been studied for such superhydrogenated molecules.

From the observations of the NW PDR of NGC 7023 we do see strong emission from the $\mathrm{CH}$ modes, and thus, we expect most PAHs to be hydrogenated at some level. Small PAHs like coronene - if present - must contribute at a negligible level to the general population of PAHs in the PDR. Coronene in the NGC 7023 NW PDR environment is the epitome of a fully dehydrogenated species. Its spectrum shows the telltale sign of graphene-like flakes with features in the 5 and $10 \mu \mathrm{m}$ region. Weak features at 5.25 and $5.75 \mu \mathrm{m}$ are routinely detected in sources whose spectra are dominated by the IR emission features (Roche et al. 1996; Boersma et al. 2009). These have been ascribed to weak overtones and combination bands involving the $\mathrm{CH}$ in-plane and out-of-plane stretching modes (Boersma et al. 2009; Mackie et al. 2015). As our study illustrates, graphene-like flakes show bands around 4.95, 5.12, and $5.35 \mu \mathrm{m}$ due to stretching modes in triple bonds (Bauschlicher \& Ricca 2013). Bands are also observed at 10 and $20 \mu \mathrm{m}$ due to in-plane stretches of the inner and outer edge $\mathrm{C}$ atoms respectively, moving to and outwards the center of the molecule. Hydrogenated PAHs do not show strong bands in the $10 \mu \mathrm{m}$ region, but can show bands in the $20 \mu \mathrm{m}$ region (Bauschlicher \& Ricca 2013).

Besides the caveat mentioned before that small graphenelike flakes are prone to loss of carbon, we should also mention that isomerization of completely dehydrogenated PAHs towards cages or rings may also be important (Berné \& Tielens 2012; Zhen et al. 2014a). This is another aspect of the evolution of PAHs in space that was not included in our modelling efforts, as kinetic data on the processes involved are lacking. Further laboratory studies are required to establish the photolysis routes of such species and to assess the origin of the 5,10, and $20 \mu \mathrm{m}$ bands in the interstellar spectra.

It is of interest to compare our model results with observations of PAH band ratios. Combining Spitzer and SOFIA observations, Croiset et al. (2016) derived 3.3/11.3 ratios in NGC 7023. This ratio is expected to serve as a proxy for PAH size. Figure 11 shows the 3.3/11.3 ratios we obtain from our model for circumcoronene and circumcircumcoronene derivatives in the NW PDR of NGC 7023. The 3.3/11.3 ratio varies slightly in our spectra due to the changes in the ionization state of the molecules. Croiset et al. (2016) report values of 0.5 to 0.3 when going from the surface of the PDR to 52 arcsec away from the star. According to our estimates, PAHs of intermediate size can reproduce such values. We do point out though, that the 3.3/11.3 is a good tracer of PAH size as long as a given ionization state dominates the distribution, e.g., at $G_{0} / n(\mathrm{H}) \sim$ 30 the 3.3/11.3 decreases for $\mathrm{C}_{96} \mathrm{H}_{24}$ species because of the dominant abundance of trications that have a lower 3.3/11.3 ratio. Therefore, beside PAH size, the observed variation in the 3.3/11.3 ratio in the NGC 7023 PDR (Croiset et al. 2016) may also be affected by charge variations.

Figure 11 also shows the variation of the 6.2/11.3 ratio in the PDR. We see the 6.2/11.3 ratio decreasing as the dominant ionization state of the PAHs changes from being $Z>0$ dominated to neutral dominated (recall that for both species the spectral variations are essentially tracing the variation in the ionization state of the parent molecules). From IRS Spitzer observations, Boersma et al. (2014) derived 6.2/11.3 ratios in the range between 1.7 and 2.0, and 7.7/11.3 ratios between 5 and 7. Fleming et al. (2010) also using IRS data reports an average value for the 7.7/11.3 of $6.06 \pm 1.74$, with the ratio varying only by $14 \%$ from a distance of $\sim 40$ to 55 arcsec from the star. Our calculated values are lower than the observed ones. Also, our 6.2/11.3 (and 7.7/11.3) band 

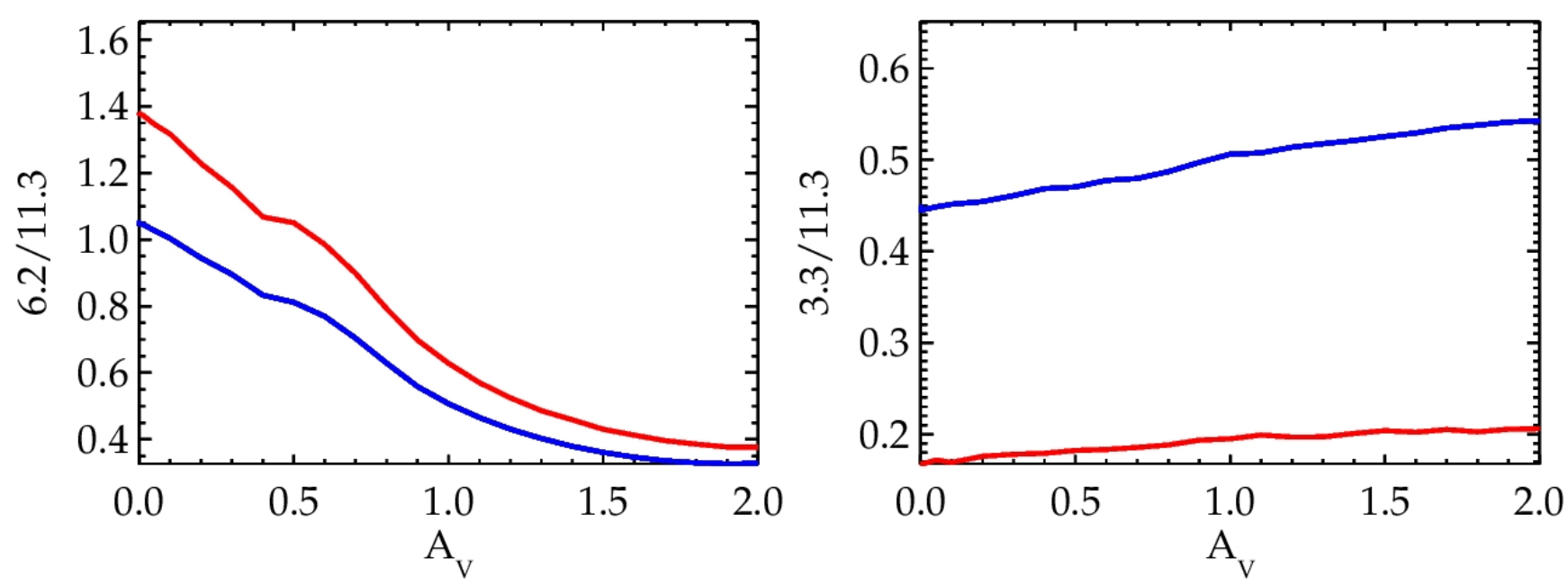

Fig. 11. PAH band ratios in the NW PDR of NGC 7023. The 6.2/11.3 ratio is usually used as a proxy for the ionization level of the PAH population, while the 3.3/11.3 ratio is expected to trace PAH size. Here we have plotted them for circumcoronene in blue, and circumcircumcoronene in red. The spectra for $\mathrm{C}_{96} \mathrm{H}_{24}$ derivatives are complete in this $A_{V}$ range. The spectra for $\mathrm{C}_{54} \mathrm{H}_{18}$ species are missing only the small contributions from partially dehydrogenated species (read text). Observational studies have derived typical 3.3/11.3 ratios of 0.5 to 0.3 from the surface to the PDR up to distances of about 52 arcsec from the star. As seen in the figure, intermediate size PAHs could potentially reproduce these values. However, the ionization ratio 6.2/11.3 would then be highly underestimated compared to the observations that predict a value of 2 at the surface of the PDR, decreasing in about $14 \%$ to a distance of $\sim 55$ arcsec from the star.

ratios vary by more than $50 \%$ from $A_{V}=0$ to $A_{V}=2$. Assuming $G_{0}=10^{3}$, we can obtain ratios close to the observed ones $\left(I_{6.2 / 11.3}\right.$ $\sim 2$, and $I_{7.7 / 11.3} \sim 6$ ) at $G_{0} / n(\mathrm{H})$ of $1-10$, instead of the $G_{0} / n(\mathrm{H})$ $\sim 0.3$ that we get at $A_{V}=0$. In other words, if the density at the surface of the cloud were overestimated by a factor of 10 , we could reproduce the observed 6.2/11.3 and 7.7/11.3 ratios with intermediate-to-large PAHs.

\subsection{Implications on the Origin of the $3.4 \mu \mathrm{m}$ feature}

Observations of Orion and NGC 7023 have both revealed bands at 3.3, 3.4, 3.47, 3.51 and $3.56 \mu \mathrm{m}$ (Joblin et al. 1996; Pilleri et al. 2015). The origin of these bands has been intensely discussed within the PAH hypothesis. The bands at $3.3 \mu \mathrm{m}$ have been widely accepted to be due to aromatic $\mathrm{CH}$ stretching modes common to the PAH family. The origin of the weaker bands (mainly the $3.4 \mu \mathrm{m}$ feature) on the other hand, still remains unknown. There are three main candidates for these carriers: (1) the bands may be hot bands of the $3.3 \mu \mathrm{m} \mathrm{CH}$ stretching mode (Barker et al. 1987); (2) the bands may be $\mathrm{CH}$ stretching modes of aliphatic side groups attached to the periphery of PAHs, such as methyl groups for example (Duley \& Williams 1981; Joblin et al. 1996); and/or (3) the bands may come from superhydrogenated PAHs, where the addition of extra $\mathrm{H}$-atoms disrupts the aromaticity of the molecule creating more bands in the region (Bernstein et al. 1996; Sandford et al. 2013).

In Sect. 4.3 (see also Appendix F) we established that, even though the spectrum of the parent molecule looks similar to those of the first 2 superhydrogenated states, the main differences reside in the $3.4 \mu \mathrm{m}$ band that appears for hydrogenation states greater than $\left(N_{\mathrm{H}}^{0}+1\right)$. This band is related to stretching modes of the pair of extra $\mathrm{H}$ atoms in the same ring. Indeed, the emission spectra from circumcoronene and circumcircumcoronene superhydrogenated species where the extra $\mathrm{H}$ atoms are added to only solo positions do not show a $3.4 \mu \mathrm{m}$ band. The $3.5 \mu \mathrm{m}$ feature instead seems to be common to the spectra of all superhydrogenated molecules. This band is related to the stretching of each individual $\mathrm{CH}_{2}$ complex. Note that here we consider superhydrogenated states of up to 4 additional $\mathrm{H}$ atoms. At these superhydrogenation levels, the $3.4 \mu \mathrm{m}$ band is only observed if the extra $\mathrm{H}$ atoms are in duo rings. For higher superhydrogenation states both, the 3.4 and $3.5 \mu \mathrm{m}$ bands, would also show up for this PAH family, since solo and duo rings would have extra $\mathrm{H}$ atoms. However such levels of superhydrogenation are achieved in extremely shielded environments, where the emission from PAHs would come from high density clumps exposed to UV radiation.

From AKARI observations of the NW PDR of NGC 7023, aside the main band observed at $3.3 \mu \mathrm{m}$ there is a secondary band at $3.4 \mu \mathrm{m}$, together with what has been defined as a plateau component at $3.45 \mu \mathrm{m}$ (Pilleri et al. 2015). The $3.4 \mu \mathrm{m}$ band appears to become less intense as the plateau widens towards more diffuse environments. Our results indicate that intermediate-tolarge PAHs can survive in their normally hydrogenated state in regions where $G_{0} / n(\mathrm{H})=10-100$, as it is expected in the more diffuse media of NGC 7023 (Berné \& Tielens 2012). It is under these conditions that the $3.3 \mu \mathrm{m}$ is still evident and the $3.4 \mu \mathrm{m}$ band starts disappearing immersed in the broadening of the $3.45 \mu \mathrm{m}$ plateau. However these PAHs would not achieve superhydrogenation. Moreover, from what we have seen, the superhydrogenation pattern of PAHs is very similar for circumcoronene and circumcircumcoronene. Thus, unless the parameters in our model are considerably changed, we would not expect $N_{\mathrm{H}}>\left(N_{\mathrm{H}}^{0}+1\right)$ species at these $G_{0} / n(\mathrm{H})$ ratios.

Pilleri et al. (2015) also report 3.4/3.3 band ratios that go from 0.028 to 0.13 from their P1 to $\mathrm{P} 4$ positions, where $G_{0}$ is expected to vary from 7000 to 150 going from the cavity into the NW PDR. If the $3.4 \mu \mathrm{m}$ band is solely due to superhydrogenated species with extra $\mathrm{H}$ atoms in duo rings, such values could be obtained at $G_{0} / n(\mathrm{H})$ ratios 2-3 orders of magnitude lower than what we expect in NGC 7023 (Fig. 12). PAHs smaller than $\mathrm{C}_{54} \mathrm{H}_{18}$ could potentially present these ratios at higher $G_{0} / n(\mathrm{H})$ of few times $10^{-2}$ at most. Nevertheless as we saw in the case of coronene, these species would probably be completely dehydrogenated, and thus the $3.4 \mu \mathrm{m}$ band would not be observed. Unless we are underestimating the density in the NW PDR of 


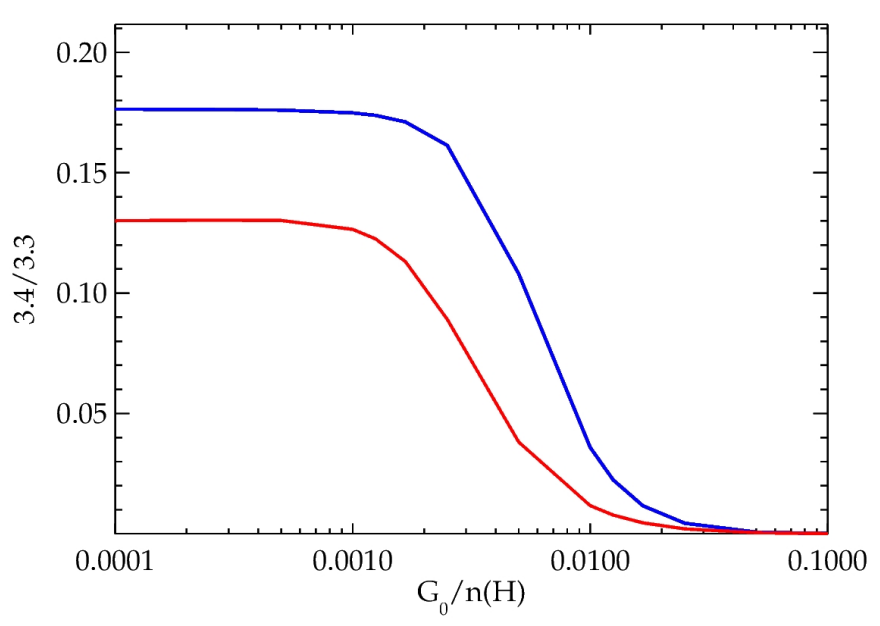

Fig. 12. Hydrogenation ratio for different $G_{0} / n(\mathrm{H})$ values. The ratios derived from circumcoronene derivatives are shown in blue, while those from circumcircumcoronene derivatives are shown in red. We have assumed a gas temperature of $50 \mathrm{~K}$, and that the fraction of atomic hydrogen is $10 \%$ that of molecular hydrogen, in order to model more shielded environments. The 3.4/3.3 ratio traces the superhydrogenation level of the PAHs as the $3.4 \mu \mathrm{m}$ band is related to the stretching modes of the pair of $\mathrm{CH}_{2}$ complexes within the same ring (which are duo rings for the coronene family). Observed 3.4/3.3 ratios in NGC 7023 range between 0.028 and 0.13 , starting from the cavity and going into the NW PDR region, where $G_{0}$ is expected to vary from 7000-150 (Pilleri et al. 2015). We see that these ratios can be achieved with superhydrogenated species but for $G_{0} / n(\mathrm{H})$ ratios of $0.002-0.006$, which is lower than what is expected in the NW PDR of NGC 7023.

NGC 7023 by a factor of 100, superhydrogenated species cannot explain the observed 3.4/3.3 ratios.

Unfortunately, other signatures of superhydrogenated species are not as clear as the appearance of bands in the $3 \mu \mathrm{m}$ region. Such species would also show aliphatic $\mathrm{CH}$ deformation modes at 6.85 and $7.25 \mu \mathrm{m}$. However these modes are intrinsically very weak and would be completely overpowered by the $\mathrm{CC}$ modes in PAH cations. Thus, we find that the main noticeable features of superhydrogenation are the $3 \mu \mathrm{m}$ bands. Longer wavelength features cannot be clearly distinguished from the ones of the parent molecules.

\section{2. $\mathrm{H}_{2}$ formation}

As mentioned in Sect. 4.2 we have considered two possible ways for PAHs to form $\mathrm{H}_{2}$ molecules in our model: either through $\mathrm{H}_{2}$ abstraction by $\mathrm{H}$, or from direct $\mathrm{H}_{2}$-loss due to photon excitation. It is of some interest then to compare the rates of these processes with those commonly adopted for interstellar dust.

Commonly, $\mathrm{H}_{2}$ is thought to form on dust grains from physisorbed $\mathrm{H}$-atoms recombining through the LangmuirHinshelwood mechanism. The standard formulation of the $\mathrm{H}_{2}$ formation rate on dust grains is then given by (Hollenbach \& McKee 1979; Burke \& Hollenbach 1983):

$$
\begin{aligned}
& R_{\mathrm{d}}\left(\mathrm{H}_{2}\right)=\frac{1}{2} S\left(T, T_{\mathrm{d}}\right) \eta \sigma_{\mathrm{d}} v_{\mathrm{th}} n(\mathrm{H}) n_{\mathrm{d}}, \\
& R_{\mathrm{d}}\left(\mathrm{H}_{2}\right) \simeq 3 \times 10^{-17} n n(\mathrm{H}) S\left(T, T_{\mathrm{d}}\right) \eta \quad \mathrm{cm}^{-3} \mathrm{~s}^{-1},
\end{aligned}
$$

where $S\left(T, T_{\mathrm{d}}\right)$ corresponds to the sticking coefficient of $\mathrm{H}$ atoms at temperature $T$ colliding with dust grains at temperature $T_{\mathrm{d}} ; \eta$ is the probability that an adsorbed $\mathrm{H}$ atom will meet another $\mathrm{H}$ atom on the surface of the grain, attach to it and be released in the form of $\mathrm{H}_{2} ; v_{\text {th }}$ is the thermal velocity of the colliding $\mathrm{H}$ atoms; $n$ is the total density; $n_{\mathrm{d}}$ is the number density of dust grains; and $\sigma_{\mathrm{d}} n_{\mathrm{d}}$ is the total cross section of dust grains. If we take $T=500 \mathrm{~K}$ and $T_{\mathrm{d}}=35 \mathrm{~K}$, the sticking coefficient is estimated to be $S \simeq 0.2$ and the $\mathrm{H}_{2}$ formation efficiency, $\eta$, is close to unity (Hollenbach \& McKee 1979; Burke \& Hollenbach 1983), resulting in $R_{\mathrm{d}}\left(H_{2}\right) \simeq 6 \times 10^{-18} \mathrm{~cm}^{3} \mathrm{~s}^{-1}$. In contrast, experiments on perfect graphite or silicate surfaces measure very low efficiencies (Pirronello et al. 1997, 1999; Katz et al. 1999) - as $\mathrm{H}$ atoms quickly evaporate before they can recombine and the inferred $\mathrm{H}_{2}$ formation rate on such surfaces is negligibly small. The models underpinning the standard dust $\mathrm{H}_{2}$ formation rate assume therefore the presence of so-called enhanced binding sites for physisorbed $\mathrm{H}$, which allow formation at much higher dust temperatures (Hollenbach \& Salpeter 1971; Hollenbach \& McKee 1979). However, the presence of enhanced binding sites is not well established and their properties are not well known. Hence, this inferred rate should perhaps more properly be considered as an upper limit.

In our analysis, we can define - analogously - the $\mathrm{H}_{2}$ formation rate through either direct $\mathrm{H}_{2}$-loss (i.e., photodissociation of PAHs) or Eley-Rideal $\mathrm{H}_{2}$ abstractions from PAHs of a given size and charge state $Z$ as:

$R_{\mathrm{PAH}(Z)}\left(\mathrm{H}_{2}\right)=\frac{1}{2} \xi_{\mathrm{H}_{2}}(Z) k_{\mathrm{col}} n_{\mathrm{PAH}(Z)}$,

where $\xi_{\mathrm{H}_{2}}(Z)$ corresponds to our previously defined efficiencies (see Sect. 4.2 ); $k_{\mathrm{col}}$ corresponds to the fiducial collision rate dependent on the charge state of the molecule (see Appendix E); and $n_{\mathrm{PAH}(Z)}$ corresponds to the number density of PAHs of a given size and charge state. From Figure 10 we conclude that the PAHs considered are not able to form $\mathrm{H}_{2}$ efficiently at the surface of the NW PDR of NGC 7023. However, considering a distribution of PAH sizes, there will always be some PAH (e.g., with $30 \lesssim N_{\mathrm{C}} \lesssim 50$ ) that could be found in the relevant hydrogenation states for $\mathrm{H}_{2}$-loss and, hence, could form $\mathrm{H}_{2}$ at efficiencies of $\sim 1 \%$ (assuming their molecular properties are similar to those of the coronene family). Assuming that $15 \%$ of the carbon is locked in PAHs (with a carbon abundance of $3.5 \times 10^{-4}$ relative to that of hydrogen), and that all PAHs have $\sim 40 \mathrm{C}$ atoms, then the $\mathrm{H}_{2}$ formation rate due to photodissociation would be a few times $10^{-19} \mathrm{~cm}^{3} / \mathrm{s}$. This is more than an order of magnitude lower than the standard rate generally adopted for dust grains, and certainly much less than the enhanced rate derived by Habart et al. (2004) for PDRs.

Bron et al. (2014) recognized the issues facing $\mathrm{H}_{2}$ formation on dust grains in PDRs, and developed a generic model describing $\mathrm{H}_{2}$ formation through the Eley-Rideal mechanism on chemisorbed $\mathrm{H}$-atoms, on a size distribution of dust grains spanning 5-3000 ̊. This is implemented in the Meudon PDR Code and was employed by us to calculate the PDR structure, albeit that we excluded the PAH-sized species in the code (cf., Table 1).

First, we recognize that our calculated efficiencies of $\sim 1 \%$ are quite comparable to those derived by Bron et al. (2014) $\left(\simeq 5 \times 10^{3}\right.$; see their Fig. 9) for conditions similar as those of NGC $7023\left(n_{\mathrm{H}}=10^{4} \mathrm{~cm}^{-3}, G_{0}=1200\right)$. This is however completely fortuitous. In their model $\mathrm{H}_{2}$ formation is an Eley-Rideal process on chemisorbed $\mathrm{H}$ atoms while in our calculations, under these conditions, the Eley-Rideal mechanism is completely unimportant given the high UV radiation field $\left(\xi_{\mathrm{H}_{2}} \sim\right.$ few times $\left.10^{-3}\right)$. In contrast, in our model, $\mathrm{H}_{2}$ formation is dominated by photolysis. There are some differences in the parameters adopted for the Eley-Rideal mechanism between our model calculations 
and those of Bron et al. (2014). The main difference is that they use an Eley-Rideal abstraction cross section that is a factor of 100 larger than the value measured for coronene films (Mennella et al. 2012) and adopted by us. Clearly, this cross section is a key factor regulating the ultimate efficiency of the $\mathrm{H}_{2}$ formation of $\mathrm{PAHs}$, and further experimental studies on free-floating PAHs will be very important to settle this issue.

Second, perusing the results of the Meudon PDR Code in more detail, the formation of $\mathrm{H}_{2}$ at low $A_{V}$ in the NW PDR of NGC 7023 is due to Eley-Rideal abstractions from dust grains with sizes larger than $\simeq 20 \AA$ (Bron et al. 2014). The calculated efficiencies for $\mathrm{H}_{2}$ formation on dust grains are of $\xi_{\mathrm{H}_{2} \text {,dust }} \simeq 0.4$ up to where the $\mathrm{HI}$ to $\mathrm{H}_{2}$ transition occurs. This boils down to an $\mathrm{H}_{2}$ formation rate about 5 times the standard value of $3 \times 10^{-17} \mathrm{~cm}^{3} / \mathrm{s}$. This is actually larger than what has been observationally inferred for a warm PDR such as NGC 7023 (Habart et al. 2004), which suggests a rate equal to the standard value. Of course, if the abstraction rate for dust grains is a factor 100 lower as experiments on films imply (Mennella et al. 2012), then the Bron et al. (2014) is a gross overestimate. Again, our model predicts that superhydrogenated PAHs are absent at the surface of the NW PDR of NGC 7023 and do not contribute to $\mathrm{H}_{2}$ formation. They only appear deep inside the cloud at $A_{V}>4.5$, where the conversion of atomic into molecular hydrogen is essentially complete.

We argue then that at the surfaces of PDRs, PAHs can contribute to the formation of $\mathrm{H}_{2}$ by means of photodissociation only if PAHs of just the "right" size dominate the PAH population in these regions. Their contribution would be then at the 1-10\% level of the standard $\mathrm{H}_{2}$ formation rate assumed for dust grains. This assumption depends very much on the kinetic parameters that control the branching ratio of $\mathrm{H}$ versus $\mathrm{H}_{2}$ photolysis channels (cf., Figs. 5 and 6), and these have been derived from experiments on the small PAH, pyrene. Further experiments on astrophysically relevant PAHs will be important to make a firm conclusion, but given this caveat, we conclude that PAHs are not the cause of the high inferred $\mathrm{H}_{2}$ formation rates in PDRs.

\section{Conclusions}

We have developed a kinetic model in order to study the abundance distribution, IR emission, and $\mathrm{H}_{2}$ formation rate for 3 specific PAHs of different sizes in the NW PDR of NGC 7023. The molecules correspond to the first 3 members of the coronene family with PAH sizes between 24 and 96 carbon atoms, which span the astrophysically relevant size range in the ISM. We have chosen to study these species at the molecular specific level, as many of their relevant molecular properties and fragmentation behaviour have been determined.

In order to model the physical conditions inside the cloud we made use of the Meudon PDR Code, and we gave this as input to our kinetic model. We considered the loss of an $\mathrm{H}$ atom or an $\mathrm{H}_{2}$ molecule as multiphoton dissociation processes, and we worked under the premise that PAHs with extra $\mathrm{H}$ atoms can form $\mathrm{H}_{2}$ through an Eley-Rideal abstraction mechanism. We then derived the abundances under the steady state assumption.

In agreement with earlier studies (Le Page et al. 2001, 2003; Montillaud et al. 2013; Boschman et al. 2012), clear differences emerge in the distribution of species with PAH size. The smallest PAH in our sample, coronene, is found to be a very fragile molecule that is easily destroyed down to its carbon cluster form (assuming no isomerization) for a wide range of $G_{0} / n(\mathrm{H})$ ratios. Only in highly shielded environments $\left(G_{0} / n(\mathrm{H})<0.01\right)$, would a small fraction of this species be found with extra $\mathrm{H}$ atoms.
The larger species on the other hand are more stable against dehydrogenation. In fact, the larger the PAH the more likely it is to find it in its normally hydrogenated state (i.e., $1 \mathrm{H}$ atom per $\mathrm{C}$ atom at its periphery). Our largest molecule, circumcircumcoronene, is mostly found in its normal hydrogenated state up to very large $G_{0} / n(\mathrm{H})$ ratios $\left(G_{0} / n(\mathrm{H}) \sim 10^{5}\right)$, while our intermediate size molecule, circumcoronene, starts losing $\mathrm{H}$ atoms at $G_{0} / n(\mathrm{H}) \gtrsim 1-10$ depending on the intensity of the UV field. Regarding the presence of PAHs with extra $\mathrm{H}$ atoms, we find that only at $G_{0} / n(\mathrm{H})<5 \times 10^{-3}$ intermediate-to-large PAHs with extra $\mathrm{H}$ atoms start dominating the abundance distribution, being in their neutral and anionic charge states.

Comparing our findings with observations of the emission in the NW PDR of NGC 7023, we find that intermediate size PAHs must be dominating the abundance of the emitting population. Small PAHs like coronene - if present - contribute at a negligible level. Together with this, we agree with previous studies on the fact that the spectral variations observed throughout the PDR in the 5-20 $\mu \mathrm{m}$ region are mainly related to variations in the ionization state of the PAHs. The footprint of hydrogenation is only discernible in the $3 \mu \mathrm{m}$ region through the specific appearance of bands at 3.4 and $3.5 \mu \mathrm{m}$. However this region could be affected by other effects such as anharmonicities or the presence of PAHs with methyl groups attached at their periphery. Based on the available AKARI observations of the NW PDR of NGC 7023, we do not expect compact PAHs with extra $\mathrm{H}$ atoms to be responsible for the 3.4/3.3 ratio observed in NGC 7023, since superhydrogenated species are only present in more shielded environments where $G_{0} / n(\mathrm{H})$ is $2-3$ orders of magnitude lower than what is derived from observations.

Finally, concerning the role of PAHs in the formation of $\mathrm{H}_{2}$ in photodissociation regions, we find that PAHs can form $\mathrm{H}_{2}$ through either Eley-Rideal abstraction of $\mathrm{H}_{2}$ from superhydrogenated PAHs in highly shielded environments, or through photodissociation under PDR-like conditions of "normal" or dehydrogenated PAHs. Our results show that, at the surface of PDRs, Eley-Rideal abstraction is never important. Our calculations are in good quantitative agreement with the model results by Bron et al. (2014) if the difference in abstraction cross section is accounted for. We find that photodissociation of PAHs can be a more important source of $\mathrm{H}_{2}$ in PDR surfaces, but this cannot account for the inferred high rates of $\mathrm{H}_{2}$ formation in PDRs, unless the kinetic parameters governing the branching ratio between $\mathrm{H}$ and $\mathrm{H}_{2}$ channels for astrophysically relevant PAHs is very different from those measured for small PAHs in the laboratory.

Acknowledgements. Studies of interstellar PAHs at Leiden Observatory are supported through advanced ERC grant 246976 from the European Research Council, through a grant by the Dutch Science Agency, NWO, as part of the Dutch Astrochemistry Network, and through the Spinoza premie from the Dutch Science Agency, NWO. A.C. acknowledges NWO for a VENI grant (grant No. 639.041.543). DFT calculations were performed with the Cartesius Supercomputer facility (grant SH-362-15).

\section{References}

Aannestad, P. A., \& Kenyon, S. J. 1979, ApJ, 230, 771

Abouelaziz, H., Gomet, J. C., Pasquerault, D., Rowe, B. R., \& Mitchell, J. B. A. 1993, J. Chem. Phys., 99, 237

Alecian, E., Catala, C., Wade, G. A., et al. 2008, MNRAS, 385, 391

Allain, T., Leach, S., \& Sedlmayr, E. 1996, A\&A, 305, 602

Allamandola, L. J., Tielens, A. G. G. M., \& Barker, J. R. 1989, ApJS, 71, 733 Allamandola, L. J., Hudgins, D. M., \& Sandford, S. A. 1999, ApJ, 511, L115 An, W., Shao, N., Bulusu, S., \& Zeng, X. C. 2008, J. Chem. Phys., 128, 084301 Andrews, H., Boersma, C., Werner, M. W., et al. 2015, ApJ, 807, 99 Bakes, E. L. O., \& Tielens, A. G. G. M. 1994, ApJ, 427, 822 
Bakes E.L.O., Tielens A.G.G.M., Bauschlicher, Jr. C.W. 2001, ApJ, 556, 501 Barker, J. R., Allamandola, L. J., \& Tielens, A. G. G. M. 1987, ApJ, 315, L61 Bauschlicher, C. W. 1998, ApJ, 509, L125

Bauschlicher, C. W., \& Bakes, E. L. O. 2001, Chem. Phys., 274, 11

Bauschlicher, C. W., \& Ricca, A. 2013, Chem. Phys. Lett., 566, 1

Bauschlicher, C. W., \& Ricca, A. 2014, Theo. Chem. Acc., 133, 1454

Bauschlicher, C. W., Peeters, E., \& Allamandola, L. J. 2008, ApJ, 678, 316

Bauschlicher, C. W., Boersma, C., Ricca, A., et al. 2010, ApJS, 189, 341

Becke, A. D. 1993, J. Chem. Phys., 98, 5648

Bernard-Salas, J., Habart, E., Köhler, M., et al. 2015, A\&A, 574, A97

Berné, O. \& Tielens, A. G. G. M. 2012, Proc. of the National Academy of Science of the US, 109, 401

Berné, O., Joblin, C., Deville, Y., et al. 2007, A\&A, 469, 575

Berné, O., Montillaud, J., Joblin, C. 2015, A\&A, 577, A133

Bernstein, M. P., Sandford, S. A., \& Allamandola, L. J. 1996, ApJ, 472, L127

Betts, N. B., Stepanovic, M., Snow, T. P., \& Bierbaum, V. M. 2006, ApJ, 651, L129

Beyer, T., \& Swinehart, D. F. 1973, Commun. Assoc. Comput. Machin. 16, 379

Biennier, L., Alsayed-Ali, M., Foutel-Richard, A., et al. 2006, Discuss. Faraday Soc., 133, 289

Boersma, C., Mattioda, A. L., Bauschlicher, C. W., et al. 2009, ApJ, 690, 1208

Boersma, C., Rubin, R. H., \& Allamandola, L. J. 2012, ApJ, 753, 168

Boersma, C., Bregman, J. D., \& Allamandola, L. J. 2013, ApJ, 769, 117

Boersma C., Bauschlicher, C. W. Jr., Ricca, A., et al. 2014, ApJS, 211, 8

Boersma, C., Bregman, J., \& Allamandola, L. J. 2015, ApJ, 806, 121

Boschman, L., Reitsma, G., Cazaux, S., et al. 2012, ApJ, 761, L33

Boschman, L., Cazaux, S., Spaans, M., Hoekstra, R., \& Schlathölter, T. 2015, A\&A, 579, A72

Bregman, J. D., Allamandola, L. J., Witteborn, F. C., Tielens, A. G. G. M., \& Geballe, T. R. 1989, ApJ, 344, 791

Bron, E., Le Bourlot, J., \& Le Petit, F. 2014, A\&A, 569, A100

Burke, J. R., \& Hollenbach, D. J. 1983, ApJ, 265, 223

Cazaux, S., Boschman, L., Rougeau, N., et al. 2016, Nature, DOI: $10.1038 /$ srep 19835

Chokshi, A., Tielens, A. G. G. M., Werner, M. W., \& Castelaz, M. W. 1988, ApJ, 334,803

Croiset, B. A., Candian, A., Berné, O., \& Tielens, A. G. G. M. 2016, A\&A, 590, A26

Cuppen, H. M., \& Herbst, E. 2005, MNRAS, 361, 565

Dawson, W. R., \& Kropp, J. L. 1969, J. Phys. Chem., 73, 693

Demarais, N. J., Yang, Z., Martinez, O., et al. 2012, AJ, 746, 32

Demarais, N. J., Yang, Z., Snow, T. P., \& Bierbaum, V. M. 2014, ApJ, 784, 25

Denifl, S., Ptasińska, S., Sonnweber, B., et al. 2005, J. Ch. Phys., 123, 104308

Draine, B. T., \& Sutin, B. 1987, ApJ, 320, 803

Duley, W. W. 1996, MNRAS, 279, 591

Duley, W. W., \& Williams, D. A. 1981, MNRAS, 196, 269

Duncan, M. A., Knight, A. M., Negishi, Y., et al. 1999, Chem. Phys. Lett., 309, 49

Fleming, B., France, K., Lupu, R. E., \& McCandliss, S. R. 2010, ApJ, 725, 159

Frisch, M. J., Trucks, G. W., Schlegel, H. B., et al. 2009, Gaussian 09, Revision D.01 (Pittsburgh PA: Gaussian, Inc.)

Fuente A., Martín-Pintado J., Rodríguez-Franco A., \& Moriarty-Schieven, G. D. 1998, A\&A 339, 575

Fuente, A., Rodríguez-Franco, A., García-Burillo, S., Martín-Pintado, J., \& Black, J. H. 2003, A\&A, 406, 899

Fusaro, M. 2012, Struct. Chem., 23, 237

Galliano, F., Madden, S. C., Tielens, A. G. G. M., Peeters, E., \& Jones, A. P. 2008, ApJ, 679, 310

Geballe, T., Tielens, A. G. G. M., Allamandola, L. J., Moorhouse, A., \& Brand, P. W. J. L. 1989, ApJ, 341, 278

Gry, C., Boulanger, F., Nehmé, C., et al. 2002, A\&A, 391, 675

Habart, E., Boulanger, F., Verstraete, L., et al. 2003, A\&A, 397, 623

Habart, E., Boulanger, F., Verstraete, L., Walmsley, C. M., \& Pineau des Forêts, G. 2004, A\&A, 414, 531

Hassouna, M., Le Garrec, J., Rebrion-Rowe, C., Travers, D., \& Rowe, B. 2003, Dissociative Recombination of Molecular Ions with Electrons, ed. S. L. Guberman (Kluwer Academic/Plenum Publishers), 451

Hollenbach, D., \& McKee, C. F. 1979, ApJS, 41, 555

Hollenbach, D., \& Salpeter, E. E. 1971, ApJ, 163, 155

Joblin, C., Tielens, A. G. G. M., Allamandola, L. J., \& Geballe, T. R. 1996, ApJ, 458,610

Joblin, C., Pilleri, P., Montillaud, J., et al. 2010, A\&A, 521, L25

Jochims, H. W., Ruhl, E., Baumgartel, H., Tobita, S., \& Leach, S. 1994, ApJ, 420, 307
Jochims, H. W., Rühl, E., Baumgärtel, H., \& Leach, S. 1996, A\&A, 314, 1003 Jochims, H. W., Rühl, E., Baumgärtel, H., Tobita, S., \& Leach, S. 1997, IJMSI, $167 / 168,35$

Jura, M. 1975, ApJ, 197, 575

Kapinus, V. 2004, Ph.D. Thesis, Caltech

Katz, N., Furman, I., Biham, O., Pirronello, V., \& Vidali, G. 1999, ApJ, 522, 305

Köhler, M., Habart, E., Arab, H., et al. 2014, A\&A, 569, A109

Kropp, J. L., \& Dawson, W. R. 1967, J. Phys. Chem., 71, 4499

Kurucz, R. L. 1993, VizieR Online Data Catalog: VI/39

Langhoff, S. R. 1996, J. Phys. Chem., 100, 2819

Le Bourlot, J., Le Petit, F., Pinto, C., Roue, E., \& Roy, F. 2012, A\&A, 541, A76 Le Page, V., Keheyan, Y., Bierbaum, V. M., \& Snow, T. P. 1997, JACS, 119, 35 Le Page, V., Snow, T. P., \& Bierbaum, V. M. 2001, ApJSS, 132, 233

Le Page, V., Snow, T. P., \& Bierbaum, V. M. 2003, AJ, 584, 316

Le Petit, F., Nehmé, C., Le Bourlot, J., \& Roueff, E. 2006, ApJSS, 164, 506

Léger, A., \& Puget, J. L. 1984, A\&A, 137, L5

Léger, A., Boissel, P., \& d'Hendecourt, L. 1988, Phys. Rev. Lett., 60, 92

Lemaire, J. L., Field, D., Gerin, M., et al. 1996, A\&A, 308, 895

Ling, Y., Gotkis, Y., \& Lifshitz, C. 1995, Eur. J. Mass Spectrom., 1, 41

Mackie, C. J., Peeters, E., Bauschlicher, C. W., Jr., \& Cami, J. 2015, ApJ, 799, 131

Malloci, G., Joblin, C., \& Mulas, G. 2007, Chem. Phys., 332, 353

Malloci, G., Mulas, G., Cecchi-Pestellini, C., \& Joblin, C. 2008, A\&A, 489, 1183

Mennella, V., Hornekær, L., Thrower, J., \& Accolla, M. 2012, ApJ, 745, L2

Montillaud, J., Joblin, C., \& Toublanc, D. 2013, A\&A, 552, A15

Novotný, O., Sivaraman, B., Rebrion-Rowe, C., et al. 2005, J. Chem. Phys., 123, 104303

Paris, C., Alcamí, M., Martín, F., \& Díaz-Tendero, S. 2014, J. Chem. Phys., 140, 204307

Peeters, E., Hony, S., Van Kerkhoven, C., et al. 2002, A\&A, 390, 1089

Pilleri, P., Montillaud, J., Berné, O., \& Joblin, C. 2012, A\&A, 542, A69

Pilleri, P., Joblin, C., Boulanger, F., \& Onaka, T. 2015, A\&A, 577, A16

Pino, T., Carpeutier, Y., Féraud, G., et al. 2011, EAS Pub. Ser., 46, 355

Pirronello, V., Biham, O., Liu, C., Shen, L., \& Vidali, G. 1997, ApJ, 483, L131

Pirronello, V., Liu, C., Roser, J. E., \& Vidali, G. 1999, A\&A, 344, 681

Press, W. H., Teukolsky, S. A., Vetterling, W. T., \& Flannery, B. P. 1992, Numerical Recipes in Fortran: The Art of Scientific Computing, 2nd edn. (Cambridge: Cambridge Univ. Press)

Purcell, E. M. 1976, ApJ, 206, 685

Radziszewski, J. G., Hess, B. A., \& Zahradnik, R. 1992, JAChS, 114, 52

Rapacioli, M., Joblin, C., \& Boissel, P. 2005, A\&A, 429, 193

Rauls, E., \& Hornekær, L. 2008, ApJ, 679, 531

Rebrion-Rowe, C., Le Garrec, J., Hassouna, M., Travers, D., \& Rowe, B. 2003, Int. Jour. Mass Spec., 223, 236

Reitsma, G., Boschman, L., Deuzeman, M.J., et al. 2014, Phys. Rev. Lett., 113, 053002

Ricca, A., Bauschlicher, C. W., Jr., Boersma, C., Tielens, A. G. G. M., \& Allamandola, L. J. 2012, ApJ, 754, 75

Ricks, A. M., Douberly, G. E., \& Duncan, M. A. 2009, ApJ, 702, 301

Roche, P. F., Lucas, P. W., Hoare, M. G., Aitken, D. K., \& Smith, C. H. 1996, MNRAS, 280, 924

Rosenberg, M. J. F., Berné, O., Boersma, C., Allamandola, L. J., \& Tielens, A. G. G. M. 2011, A\&A, 532, A128

Sandford, S. A., Bernstein, M. P., \& Materese, C. K. 2013, ApJS, 205, 8

Schutte, W. A., Tielens, A. G. G. M., \& Allamandola, L. J. 1993, ApJ, 415, 397

Sellgren, K., Werner, M. W., Ingalls, J. G., et al. 2010, ApJ, 722, L54

Stein, S. E., \& Rabinovitch, B. S., 1973, J. Phys. Chem., 58, 2438

Stephens, P. J., Devlin, F. J., Chabalowski, C. F., \& Frisch, M. J. 1994, J. Phys. Chem., 98, 11623

Tielens, A. G. G. M. 2005, The Physics and Chemistry of the Interstellar Medium (Cambridge: Cambridge Univ. Press)

Tielens, A. G. G. M. 2008, ARA\&A, 46, 289

Thrower, J. D., Nilsson, L., Jørgensen, B., et al. 2011, in PAHs and the Universe, eds. C. Joblin, \& A. G. G. M. Tielens (Les Ulis: EDP), EAS Pub. Ser. 46, 453

Tobita, S., Meinke, M., Illenberger, E., et al. 1992, Chem. Phys., 161, 501

Tobita, S., Leach, S., Jochims, H. W., et al. 1994, Canadian J. Phys., in press

Verstraete, L., Leger, A., d'Hendecourt, L., Defourneau, D., \& Dutuit, O. 1990, A\&A, 237, 436

Werner, M. W., Uchida, K. I., Sellgren, K., et al. 2004, ApJS, 154, 309

Witt, A. N., Gordon, K. D., Vijh, U. P., et al. 2006, ApJ, 636, 303

Young Owl, R. C., Meixner, M. M., Fong, D., et al. 2002, ApJ, 578, 885

Zhen, J., Castellanos, P., Paardekooper, D. M., Linnartz, H., \& Tielens, A. G. G. M. 2014a, ApJ, 797, L30 
Table A.1. Ionization potentials and electron affinities of $\mathrm{C}_{24} \mathrm{H}_{12}$, $\mathrm{C}_{54} \mathrm{H}_{18}$ and $\mathrm{C}_{96} \mathrm{H}_{24}$.

\begin{tabular}{lccc}
\hline \hline Molecule & & $\begin{array}{c}\text { Ionization potentials } \\
\text { Energy (eV) }\end{array}$ & References \\
\hline $\mathrm{C}_{24} \mathrm{H}_{12}$ & IP(1) & 7.20 & Tobita et al. (1994) \\
& IP(2) & 11.50 & Tobita et al. (1994) \\
\hline $\mathrm{C}_{54} \mathrm{H}_{18}$ & IP(1) & 6.14 & Malloci et al. (2007) \\
& IP(2) & 8.91 & Malloci et al. (2007) \\
& IP(3) & 12.94 & Bakes \& Tielens (1994) \\
& IP(1) & 5.68 & Bakes \& Tielens (1994) \\
$\mathrm{C}_{96} \mathrm{H}_{24}$ & $\mathrm{IP}(2)$ & 8.24 & Bakes \& Tielens (1994) \\
& IP(3) & 10.80 & Bakes \& Tielens (1994) \\
& IP(4) & 13.36 & Bakes \& Tielens (1994) \\
\hline \hline & & Electron Affinities & \\
$\mathrm{Molecule}$ & & Energy (eV) & References \\
\hline $\mathrm{C}_{24} \mathrm{H}_{12}$ & EA(1) & 0.47 & Duncan et al. (1999) \\
\hline $\mathrm{C}_{54} \mathrm{H}_{18}$ & EA(1) & 1.44 & Malloci et al. (2007) \\
\hline $\mathrm{C}_{96} \mathrm{H}_{24}$ & EA(1) & 0.56 & Bakes \& Tielens (1994) \\
& EA(2) & 3.11 & Bakes \& Tielens (1994) \\
\hline
\end{tabular}

\section{Appendix A: Molecular characteristics}

\section{A.1. Ionization potentials and electron affinities}

Malloci et al. (2008) calculated the IPs for the even dehydrogenated states of coronene, i.e., $\mathrm{C}_{24} \mathrm{H}_{2 n}$ where $n=0-6$. According to their calculations, the IP increases with higher levels of dehydrogenation. This increase is relatively constant, with $\Delta \mathrm{IP} \sim 0.1 \mathrm{eV}$ per dehydrogenation (see Fig. 4). For coronene, the theoretically calculated energies vary from $7.02 \mathrm{eV}$ for $\mathrm{C}_{24} \mathrm{H}_{12}$ to $8.25 \mathrm{eV}$ for $\mathrm{C}_{24}$. This last value also agrees with the calculations of An et al. (2008) for $\mathrm{C}_{24}$. The decrease of the IP with hydrogenation has also been reported for anthracene $\left(\mathrm{C}_{14} \mathrm{H}_{10}\right)$ and naphthalene $\left(\mathrm{C}_{10} \mathrm{H}_{8}\right)$ first dehydrogenated and first superhydrogenated states, where $\triangle \mathrm{IP}$ becomes more constant as the PAH gets larger (Kapinus 2004). Based on this, we decided to adopt this $\Delta$ IP with hydrogenation for all molecules and all ionization states.

\section{A.2. IR intrinsic spectra}

Tables A.2-A.4 list the PAHs for which we have the IR intrinsic spectra. The spectra are either taken from the NASA Ames PAH Database (PAHdb) or from our own calculations (C).

\section{A.3. Electron attachment/recombination with electrons}

Tobita et al. (1992) performed low energy electron impact experiments on small neutral PAHs, and reported rate coefficients for electron attachment of $9 \times 10^{-10}$ and $2 \times 10^{-10} \mathrm{~cm}^{3} / \mathrm{s}$ for anthracene $\left(\mathrm{C}_{14} \mathrm{H}_{10}\right)$ and pyrene $\left(\mathrm{C}_{16} \mathrm{H}_{10}\right)$, respectively. Denifl et al. (2005) studied the electron attachment to neutral coronene and corannulene $\left(\mathrm{C}_{20} \mathrm{H}_{10}\right)$, and obtained similar trends as observed in the experiments of Tobita et al. (1992). They estimated the electron recombination cross section to be of $3.8 \times 10^{-16} \mathrm{~cm}^{2}$ for the formation of the coronene anion. Regarding cations, electron recombination rates have been measured for a few small PAH species using flowing afterglow experiments. These studies have determined rate coefficients of $1 \times 10^{-6}$ for benzene, $3 \times 10^{-7}$ for naphthalene, $1.1 \times 10^{-6}$ for azulene, $5.0 \times 10^{-7}$ for acenaphthene, $2.4 \times 10^{-6}$ for anthracene, phenanthrene and fluoranthene, and $4.1 \times 10^{-6} \mathrm{~cm}^{3} / \mathrm{s}$ for pyrene (Abouelaziz et al. 1993;
Table A.2. PAH Intrinsic spectra available for coronene $\mathrm{C}_{24} \mathrm{H}_{12}$ derivatives.

\begin{tabular}{|c|c|c|c|c|}
\hline$N_{\mathrm{H}}$ & $Z=-1$ & $Z=0$ & $Z=1$ & $Z=2$ \\
\hline 0 & $\ldots$ & PAHdb & PAHdb & $\mathrm{C}$ \\
\hline 1 & $\ldots$ & C & C & C \\
\hline 2 & $\ldots$ & $\mathrm{C}$ & $\mathrm{C}$ & $\mathrm{C}$ \\
\hline 3 & $\ldots$ & $\mathrm{C}$ & $\mathrm{C}$ & $\mathrm{C}$ \\
\hline 4 & $\ldots$ & $\ldots$ & $\mathrm{C}$ & $\mathrm{C}$ \\
\hline 5 & $\ldots$ & $\ldots$ & $\mathrm{C}$ & $\mathrm{C}$ \\
\hline 6 & $\ldots$ & $\ldots$ & $\mathrm{C}$ & $\mathrm{C}$ \\
\hline 7 & $\ldots$ & $\ldots$ & $\mathrm{C}$ & $\mathrm{C}$ \\
\hline 8 & $\ldots$ & $\ldots$ & $\mathrm{C}$ & $\mathrm{C}$ \\
\hline 9 & $\ldots$ & $\ldots$ & $\mathrm{C}$ & $\mathrm{C}$ \\
\hline 10 & $\ldots$ & $\ldots$ & C & C \\
\hline 11 & & & $\mathrm{C}$ & $\mathrm{C}$ \\
\hline 12 & PAHdb & PAHdb & PAHdb & PAHdb \\
\hline 13 & PAHdb & C & C & C \\
\hline 14 & C & $\mathrm{C}$ & $\mathrm{C}$ & $\mathrm{C}$ \\
\hline
\end{tabular}

Table A.3. PAH Intrinsic spectra available for circumcoronene $\mathrm{C}_{54} \mathrm{H}_{18}$ derivatives.

\begin{tabular}{llcccc}
\hline \hline$N_{\mathrm{H}}$ & $Z=-1$ & $Z=0$ & $Z=1$ & $Z=2$ & $Z=3$ \\
\hline 0 & $\ldots$ & PAHdb & PAHdb & $\ldots$ & $\ldots$ \\
1 & $\ldots$ & $\ldots$ & $\mathrm{C}$ & $\ldots$ & $\ldots$ \\
17 & $\ldots$ & $\mathrm{C}$ & $\ldots$ & $\ldots$ & $\ldots$ \\
18 & PAHdb & PAHdb & PAHdb & PAHdb & PAHdb \\
19 & PAHdb & $\mathrm{C}$ & PAHdb & PAHdb & PAHdb \\
20 & $\mathrm{C}$ & $\mathrm{C}$ & $\ldots$ & $\ldots$ & $\ldots$ \\
\hline
\end{tabular}

Notes. No spectra are available for $N_{\mathrm{H}}=2-16$.

Table A.4. PAH Intrinsic spectra available for circumcircumcoronene $\mathrm{C}_{96} \mathrm{H}_{24}$ derivatives.

\begin{tabular}{llcccccc}
\hline \hline$N_{\mathrm{H}}$ & $Z=-2$ & $Z=-1$ & $Z=0$ & $Z=1$ & $Z=2$ & $Z=3$ & $Z=4$ \\
\hline 0 & $\ldots$ & $\ldots$ & PAHdb & PAHdb & PAHdb & $\ldots$ & $\ldots$ \\
22 & $\ldots$ & PAHdb & PAHdb & PAHdb & $\ldots$ & $\ldots$ & $\ldots$ \\
24 & PAHdb & PAHdb & PAHdb & PAHdb & PAHdb & PAHdb & PAHdb \\
25 & $\ldots$ & PAHdb & PAHdb & PAHdb & $\ldots$ & $\ldots$ & $\ldots$ \\
26 & $\ldots$ & $\mathrm{C}$ & $\mathrm{C}$ & $\ldots$ & $\ldots$ & $\ldots$ & $\ldots$ \\
\hline
\end{tabular}

Notes. No spectra are available for $N_{\mathrm{H}}=1-21$.

Hassouna et al. 2003; Rebrion-Rowe et al. 2003; Novotný et al. 2005; Biennier et al. 2006). No studies have been done for the PAHs considered here.

Given the lack of experimental studies on large PAHs, we use collisional rates as described in Bakes \& Tielens (1994), which take into account the Coulomb interaction between the particles involved:

$k_{\mathrm{rec}}\left(Z, N_{\mathrm{H}}\right)=n(e) s_{\mathrm{e}}\left(Z, N_{\mathrm{H}}\right)\left(\frac{8 k_{\mathrm{B}} T_{\mathrm{gas}}}{\pi m_{\mathrm{e}}}\right)^{1 / 2} \pi a^{2} \tilde{J}$,

where $n(e)$ is the electron density, $k_{\mathrm{B}}$ is the Boltzmann constant, $T_{\text {gas }}$ the gas temperature, $m_{\mathrm{e}}$ the electron mass, $s_{\mathrm{e}}\left(Z, N_{\mathrm{H}}\right)$ is the electron sticking coefficient for each molecule, $a$ is the radius of the $\mathrm{PAH}$, and $\tilde{J}$ is a function that depends on the electron charge and the charge of the PAH (see full expression in Draine \& Sutin 1987). We calculate the radius of each molecule as $a=0.9 \times 10^{-8} N_{\mathrm{C}}^{1 / 2} \mathrm{~cm}$, where $N_{\mathrm{C}}$ is the number of carbon atoms of the molecule. Since Eq. (A.1) is valid for spherical molecules, we use a 0.82 correction factor as derived by Verstraete et al. (1990) for disk-shaped molecules. For positively ionized molecules we assume a sticking coefficient of unity given the attractive interaction between the particles. Sticking coefficients for neutral species are calculated following Allamandola et al. (1989). Polarizabilities are taken from 
Table B.1. Fit parameters to $\mathrm{H}$-loss rate, $k_{\mathrm{H} \text {,Loss }}$, and $\mathrm{H}_{2}$-loss rate, $k_{\mathrm{H}_{2} \text {,Loss }}$, as a function of UV field intensity $G_{0}$ for coronene derivatives.

\begin{tabular}{|c|c|c|c|c|c|c|c|c|c|c|}
\hline \multicolumn{6}{|c|}{ H-Loss } & \multicolumn{5}{|c|}{$\mathrm{H}_{2}$-Loss } \\
\hline$N_{\mathrm{H}}$ & $p_{0}$ & $p_{1}$ & $p_{2}$ & $p_{3}$ & $p_{4}$ & $p_{0}$ & $p_{1}$ & $p_{2}$ & $p_{3}$ & $p_{4}$ \\
\hline \multicolumn{11}{|c|}{$Z=-1$} \\
\hline 12 & -6.131 & 1.154 & -0.153 & 0.038 & -0.001 & -8.163 & 0.909 & 0.119 & -0.047 & 0.006 \\
\hline 13 & 3.405 & 0.999 & 0.001 & -0.001 & 0.000 & $\ldots$ & $\cdots$ & $\ldots$ & $\ldots$ & .. \\
\hline 14 & 3.448 & 0.999 & 0.004 & -0.002 & 0.000 & $\ldots$ & $\ldots$ & $\ldots$ & $\ldots$ & \\
\hline \multicolumn{11}{|c|}{$Z=0$} \\
\hline 1 & -3.814 & 1.083 & -0.065 & 0.008 & 0.002 & $\cdots$ & & $\ldots$ & .. & \\
\hline 2 & -5.820 & 1.198 & -0.206 & 0.057 & -0.003 & -7.870 & 0.933 & 0.096 & -0.042 & 0.006 \\
\hline 3 & -1.850 & 0.947 & 0.083 & -0.039 & 0.006 & $\ldots$ & $\ldots$ & $\ldots$ & $\ldots$ & \\
\hline 12 & -5.800 & 1.192 & -0.198 & 0.054 & -0.003 & -7.849 & 0.925 & 0.104 & -0.043 & 0.006 \\
\hline 13 & 3.791 & 1.001 & 0.004 & -0.002 & 0.000 & $\ldots$ & $\ldots$ & $\ldots$ & $\ldots$ & $\ldots$ \\
\hline 14 & 3.693 & 0.997 & 0.005 & -0.003 & 0.000 & $\ldots$ & $\ldots$ & $\ldots$ & $\ldots$ & $\ldots$ \\
\hline \multicolumn{11}{|c|}{$Z=1$} \\
\hline 1 & -2.082 & 1.166 & -0.014 & -0.019 & 0.004 & $\ldots$ & & $\cdots$ & $\ldots$ & \\
\hline 2 & -3.901 & 0.960 & 0.064 & -0.031 & 0.005 & -6.494 & 0.907 & 0.116 & -0.043 & 0.005 \\
\hline 3 & -2.359 & 0.917 & 0.109 & -0.043 & 0.005 & $\ldots$ & & & $\ldots$ & \\
\hline 4 & -4.356 & 0.986 & 0.038 & -0.023 & 0.004 & -6.866 & 0.898 & 0.136 & -0.050 & 0.006 \\
\hline 5 & -2.698 & 0.931 & 0.096 & -0.041 & 0.005 & .. & & & $\ldots$ & \\
\hline 6 & -4.083 & 1.087 & -0.060 & 0.005 & 0.001 & -6.592 & 0.892 & 0.128 & -0.045 & 0.005 \\
\hline 7 & -3.135 & 1.076 & -0.044 & 0.000 & 0.002 & 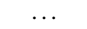 & .. & $\ldots$ & $\ldots$ & \\
\hline 8 & -5.225 & 1.143 & -0.130 & 0.028 & 0.000 & -7.481 & 0.900 & 0.126 & -0.047 & 0.006 \\
\hline 9 & -3.540 & 0.962 & 0.064 & -0.031 & 0.005 & $\ldots$ & $\ldots$ & $\ldots$ & $\ldots$ & \\
\hline 10 & -5.728 & 1.108 & -0.088 & 0.017 & 0.001 & -7.884 & 0.900 & 0.139 & -0.051 & 0.006 \\
\hline 11 & -3.929 & 1.020 & 0.030 & -0.023 & 0.004 & $\ldots$ & $\ldots$ & $\ldots$ & $\ldots$ & \\
\hline 12 & -6.106 & 1.151 & -0.150 & 0.037 & -0.001 & -8.155 & 0.912 & 0.114 & -0.044 & 0.005 \\
\hline 13 & 3.254 & 1.047 & -0.021 & 0.004 & 0.000 & $\ldots$ & $\ldots$ & $\cdots$ & $\ldots$ & $\cdots$ \\
\hline 14 & 3.007 & 0.998 & 0.004 & -0.002 & 0.000 & $\ldots$ & $\ldots$ & $\ldots$ & $\ldots$ & $\ldots$ \\
\hline
\end{tabular}

the Cagliari PAH database for the parent molecules $\mathrm{C}_{24} \mathrm{H}_{12}$ and $\mathrm{C}_{54} \mathrm{H}_{18}$. To determine the polarizability of $\mathrm{C}_{96} \mathrm{H}_{24}$, we performed a fit as a function of size to the polarizabilities of all the PAHs compiled in the database (up to sizes of $54 \mathrm{C}$ atoms) for a given ionization state, and then extrapolated this to $N_{\mathrm{C}}=96$. We then used the polarizabilities of the parent molecules for all the respective derivatives. The calculated sticking coefficients increase with EA, reaching unity for EA $\gtrsim 1 \mathrm{eV}$.

\section{Appendix B: Photodissociation rates}

Tables B.1-B.3 present the fit parameters to the H-loss and $\mathrm{H}_{2}$-loss rates as a function of UV field intensity $G_{0}$ for the molecules considered in our work. The fits are of the form $\log _{10}\left(k_{i}\left(G_{0}\right)\right)=\sum_{j} p_{j} \log _{10}\left(G_{0}\right)^{j}$ from $j=0-4$ for each $i$ process (i.e., $\mathrm{H}$-loss and $\mathrm{H}_{2}$-loss). The fits are shown for the molecules for which the IR intrinsic spectra were available.

\section{Appendix C: Model}

Since there are $\left(N_{\mathrm{H}}^{0}+5\right)$ hydrogenation states for each parent molecule, and $N_{Z}$ ionization states depending on the molecule, for each species we must solve $\left(N_{\mathrm{H}}^{0}+5\right) \times N_{Z}$ equations of the form:

$$
\frac{\mathrm{d}\left[\mathrm{PAH}\left(Z, N_{\mathrm{H}}\right)\right]}{\mathrm{d} t}=\sum(\text { Formation })-\sum(\text { Destruction }),
$$

where formation processes (when applicable) include: ionization of PAH $\left(Z-1, N_{\mathrm{H}}\right)$; electron attachment to PAH $\left(Z+1, N_{\mathrm{H}}\right)$; loss of an $\mathrm{H}$ atom from PAH $\left(Z, N_{\mathrm{H}}+1\right)$; loss of $\mathrm{H}_{2}$ from PAH $(Z$, $\left.N_{\mathrm{H}}+2\right)$; $\mathrm{H}$ addition to PAH $\left(Z, N_{\mathrm{H}}-1\right)$ (or PAH $\left(Z-1, N_{\mathrm{H}}-1\right)$ in the case of associative detachment); and $\mathrm{H}_{2}$ abstraction from $\mathrm{PAH}\left(Z, N_{\mathrm{H}}+1\right)$. Destruction processes on the other hand (when applicable) are given by: ionization of PAH $\left(Z, N_{\mathrm{H}}\right)$; electron
Table B.2. Fit parameters to $\mathrm{H}$-loss rate, $k_{\mathrm{H}, \mathrm{Loss}}$, and $\mathrm{H}_{2}$-loss rate, $k_{\mathrm{H}_{2} \text {,Loss }}$, as a function of UV field intensity $G_{0}$ for circumcoronene derivatives.

\begin{tabular}{|c|c|c|c|c|c|c|c|c|c|c|}
\hline \multicolumn{6}{|c|}{ H-Loss } & \multicolumn{5}{|c|}{$\mathrm{H}_{2}$-Loss } \\
\hline$N_{\mathrm{H}}$ & $p_{0}$ & $p_{1}$ & $p_{2}$ & $p_{3}$ & $p_{4}$ & $p_{0}$ & $p_{1}$ & $p_{2}$ & $p_{3}$ & $p_{4}$ \\
\hline \multicolumn{11}{|c|}{$Z=-1$} \\
\hline 18 & -13.972 & 0.541 & 0.498 & -0.087 & 0.007 & -14.226 & 1.179 & -0.231 & 0.079 & -0.005 \\
\hline 19 & -2.676 & 0.973 & 0.040 & -0.017 & 0.003 & $\ldots$ & $\ldots$ & $\ldots$ & $\ldots$ & $\ldots$ \\
\hline 20 & -2.866 & 1.275 & -0.130 & 0.019 & 0.000 & $\ldots$ & $\ldots$ & $\ldots$ & $\ldots$ & $\ldots$ \\
\hline \multicolumn{11}{|c|}{$Z=0$} \\
\hline 17 & -10.438 & 0.597 & 0.375 & -0.058 & 0.005 & & & & & \\
\hline 18 & -14.148 & 1.962 & -0.031 & -0.009 & 0.003 & -13.527 & 1.051 & -0.121 & 0.060 & -0.004 \\
\hline 19 & -0.624 & 0.979 & 0.031 & -0.014 & 0.002 & $\ldots$ & $\ldots$ & $\ldots$ & $\ldots$ & $\ldots$ \\
\hline 20 & -0.982 & 1.228 & -0.115 & 0.021 & -0.001 & $\ldots$ & $\ldots$ & $\ldots$ & $\ldots$ & $\ldots$ \\
\hline \multicolumn{11}{|c|}{$Z=1$} \\
\hline 1 & -10.821 & 0.825 & 0.101 & 0.015 & -0.001 & & & & & \\
\hline 18 & -13.752 & 0.537 & 0.503 & -0.089 & 0.007 & -14.036 & 1.179 & -0.230 & 0.078 & -0.005 \\
\hline 19 & -2.904 & 0.961 & 0.058 & -0.026 & 0.004 & $\ldots$ & $\ldots$ & $\ldots$ & $\ldots$ & $\ldots$ \\
\hline
\end{tabular}

Table B.3. Fit parameters to $\mathrm{H}$-loss rate, $k_{H, L o s s}$, and $\mathrm{H}_{2}$-loss rate, $k_{H_{2} \text {,Loss }}$, as a function of UV field intensity $G_{0}$ for circumcircumcoronene derivatives.

\begin{tabular}{|c|c|c|c|c|c|c|c|c|c|c|}
\hline \multicolumn{6}{|c|}{ H-Loss } & \multicolumn{5}{|c|}{$\mathrm{H}_{2}$-Loss } \\
\hline$N_{\mathrm{H}}$ & $p_{0}$ & $p_{1}$ & $p_{2}$ & $p_{3}$ & $p_{4}$ & $p_{0}$ & $p_{1}$ & $p_{2}$ & $p_{3}$ & $p_{4}$ \\
\hline \multicolumn{11}{|c|}{$Z=-1$} \\
\hline 22 & -20.455 & 1.746 & 0.265 & -0.098 & 0.012 & -19.627 & 0.667 & 0.429 & -0.068 & 0.005 \\
\hline 24 & -20.666 & 1.769 & 0.254 & -0.096 & 0.012 & -19.841 & 0.812 & 0.330 & -0.043 & 0.003 \\
\hline 25 & -5.927 & 0.859 & 0.161 & -0.066 & 0.010 & $\ldots$ & $\ldots$ & $\ldots$ & $\ldots$ & $\ldots$ \\
\hline 26 & -6.042 & 0.990 & 0.045 & -0.033 & 0.007 & $\ldots$ & & $\ldots$ & $\ldots$ & \\
\hline \multicolumn{11}{|c|}{$Z=0$} \\
\hline 22 & -19.161 & 1.960 & 0.050 & -0.029 & 0.007 & -18.846 & 0.693 & 0.679 & -0.167 & 0.016 \\
\hline 24 & -19.318 & 2.106 & -0.074 & 0.005 & 0.004 & -19.040 & 1.019 & 0.428 & -0.100 & 0.010 \\
\hline 25 & -2.764 & 0.820 & 0.223 & -0.088 & 0.012 & $\ldots$ & $\ldots$ & $\ldots$ & $\ldots$ & $\ldots$ \\
\hline 26 & -2.819 & 0.858 & 0.186 & -0.076 & 0.011 & $\ldots$ & $\ldots$ & $\ldots$ & $\ldots$ & $\ldots$ \\
\hline \multicolumn{11}{|c|}{$Z=1$} \\
\hline 22 & -20.559 & 1.741 & 0.236 & -0.085 & 0.011 & -19.491 & 0.420 & 0.498 & -0.075 & $\overline{0.005}$ \\
\hline 24 & -20.700 & 1.802 & 0.181 & -0.066 & 0.009 & -19.818 & 0.795 & 0.269 & -0.016 & 0.000 \\
\hline 25 & -6.489 & 0.910 & 0.136 & -0.059 & 0.009 & ... & & $\ldots$ & $\ldots$ & $\ldots$ \\
\hline
\end{tabular}

attachment to PAH $\left(Z, N_{\mathrm{H}}\right)$; loss of an $\mathrm{H}$ atom from PAH $(Z$, $\left.N_{\mathrm{H}}\right)$; loss of $\mathrm{H}_{2}$ from PAH $\left(Z, N_{\mathrm{H}}\right) ; \mathrm{H}$ addition to PAH $\left(Z, N_{\mathrm{H}}\right)$; and $\mathrm{H}_{2}$ abstraction from PAH $\left(Z, N_{\mathrm{H}}\right)$.

The system of equations can then be written in matrix form as $M \boldsymbol{x}=\mathbf{0}$, where $M$ represents the kinetic matrix with all the rate constants calculated for a given set of physical conditions $T_{\text {gas }}, n(e), n(\mathrm{H})$ and $G_{0}$. For each slab of the PDR this system of equations is solved using the Newton-Raphson Method (Press et al. 1992). This method proved to be fast and reached convergence for every slab considered in our work.

\section{Appendix D: Relative abundance distribution of PAHs in NGC 7023}

Figures D.1-D.3 show the fractional contribution from coronene (black), circumcoronene (blue) and circumcircumcoronene (red) derivatives in the NW PDR of NGC 7023, according to the physical conditions modelled by the Meudon PDR Code. Odd and even hydrogenated states have been depicted in slightly different colors for a better visualization.

Coronene is completely dehydrogenated throughout most of the cloud $\left(N_{\mathrm{H}}=0\right)$. Only at an $A_{V}>4$ we find traces of the first 4 hydrogenated states. This is driven by a rise in the atomic hydrogen density due to the modelling of the cloud (finite size). 
H. Andrews et al.: Hydrogenation and dehydrogenation of interstellar PAHs: Spectral characteristics and $\mathrm{H}_{2}$ formation
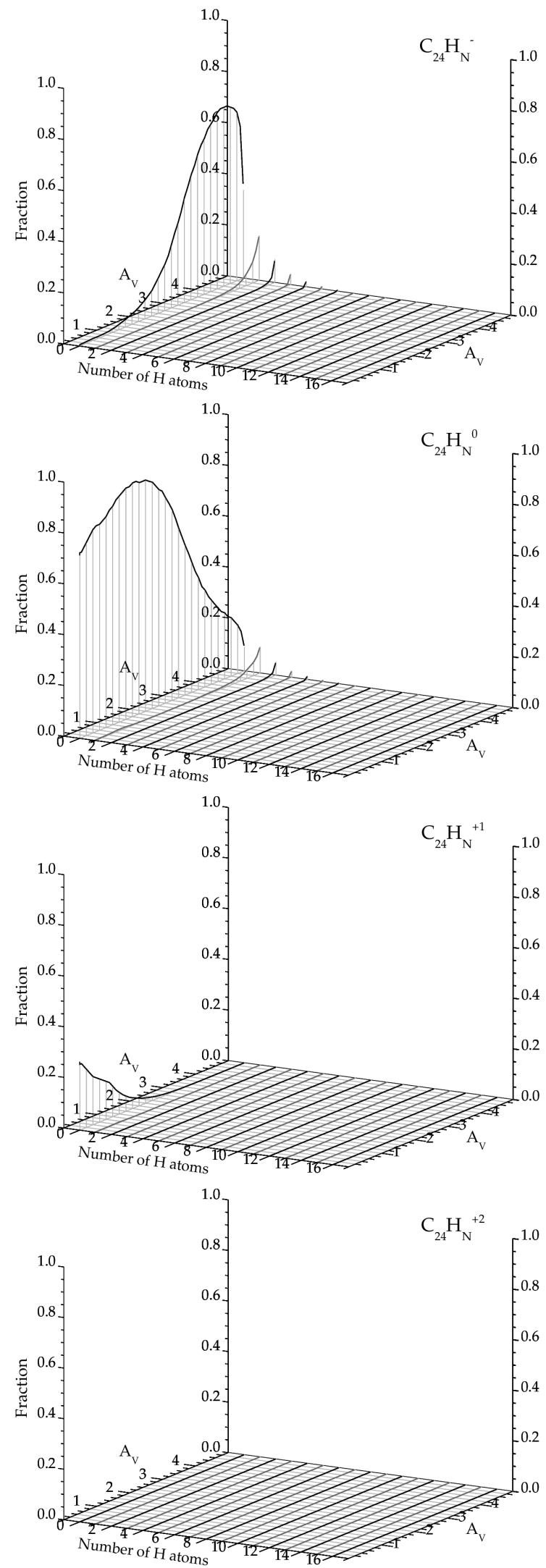

Fig. D.1. Fractional contribution from coronene derivatives in the NW PDR of NGC 7023 according to the physical conditions modelled by the Meudon PDR Code. Coronene is completely dehydrogenated throughout most of the cloud $\left(N_{\mathrm{H}}=0\right)$. No contribution from dicationic species is observed.
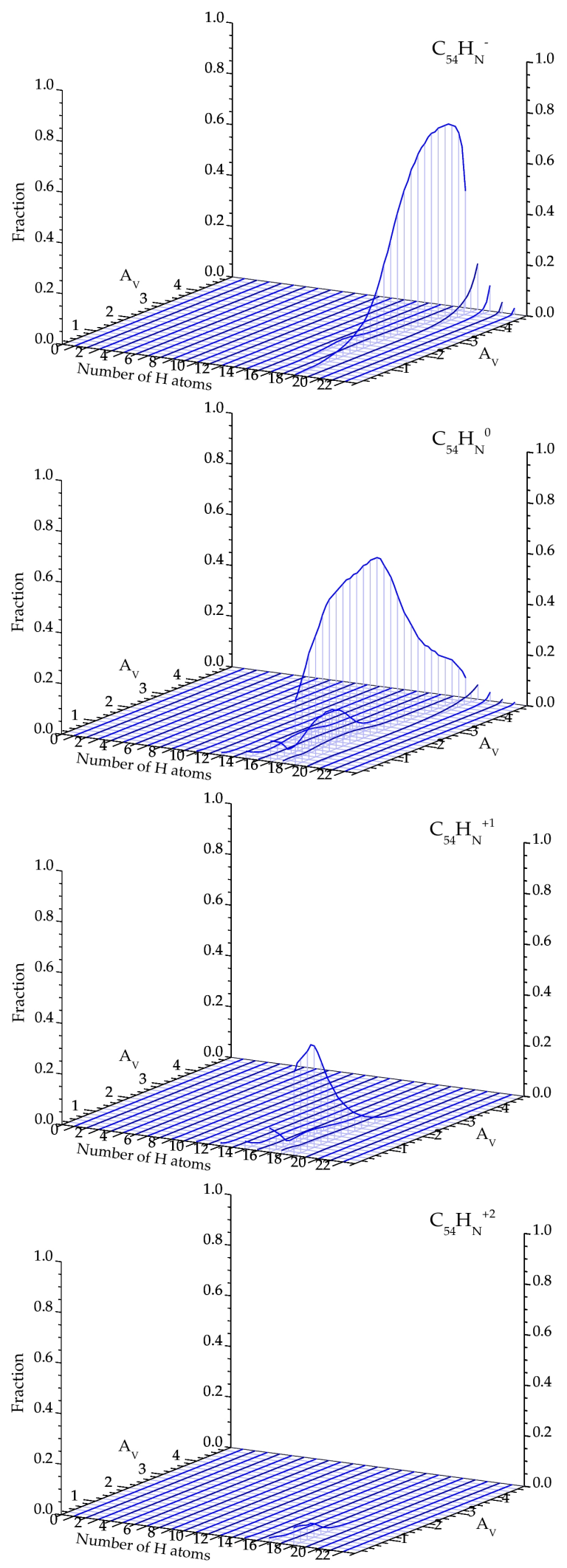

Fig. D.2. Fractional contribution from circumcoronene derivatives in the NW PDR of NGC 7023 according to the physical conditions modelled by the Meudon PDR Code. Unlike coronene, these molecules are found in their normally hydrogenated state $\left(N_{\mathrm{H}}=18\right)$ with traces of partial dehydrogenation at the surface of the cloud, and at $A_{V} \sim 1.8$. No contribution from trications is observed. 

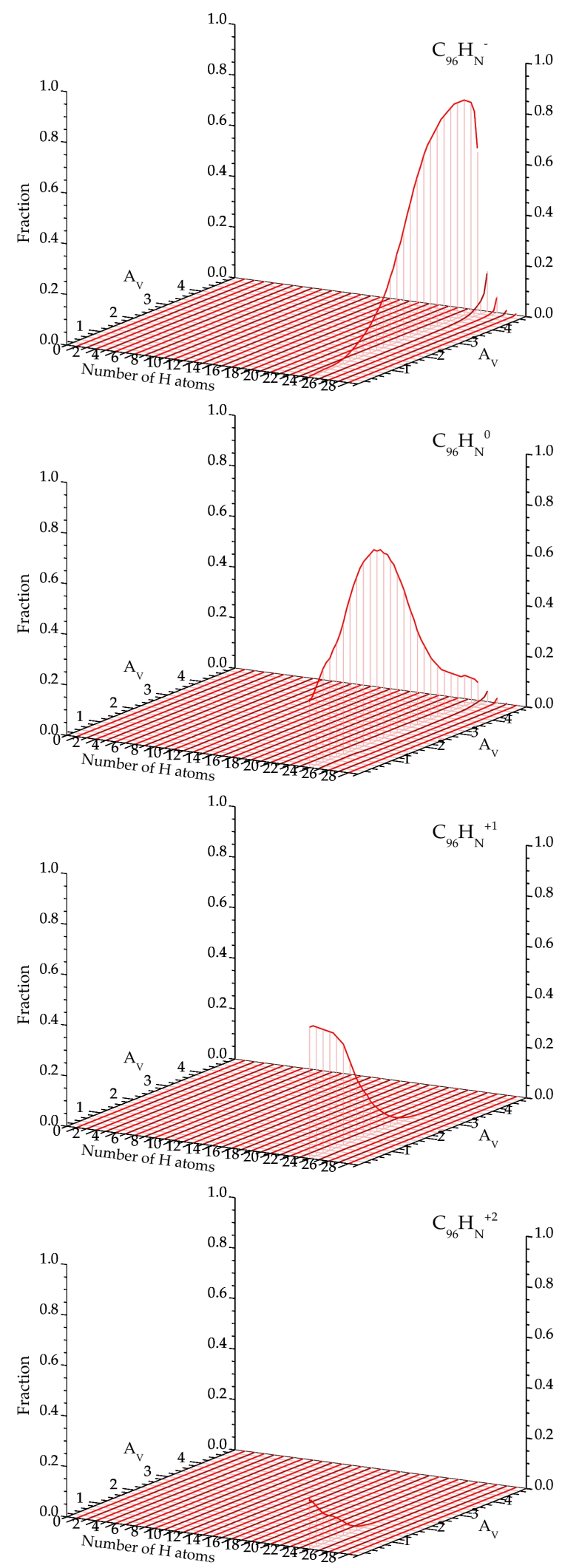

Fig. D.3. Fractional contribution from circumcircumcoronene derivatives in the NW PDR of NGC 7023 according to the physical conditions modelled by the Meudon PDR Code. Compared to the smaller species, circumcircumcoronene is mainly found in its normal hydrogenated state $\left(N_{\mathrm{H}}=24\right)$. There is no significant contribution from $Z>2$ species (trications contribute in only $0.9 \%$ at $A_{V}=0$ ).
In terms of the ionization state of the coronene derivatives, we find that at the surface of the cloud $\left(A_{V}=0\right)$ most molecules are in neutral state, while cations contribute in $\sim 27 \%$ to the total PAH abundance.

Unlike coronene, circumcoronene derivatives are found in their normally hydrogenated state $\left(N_{\mathrm{H}}=18\right)$ with traces of partial dehydrogenation at the surface of the cloud, and at $A_{V} \sim$ 1.8. Regarding ionization, positively ionized species dominate the distribution at the surface of the cloud, contributing in $\sim 59 \%$ to the total PAH abundance.

Compared to the smaller molecules, circumcircumcoronene derivatives are mainly found in the normal hydrogenated state $\left(N_{\mathrm{H}}=24\right)$. No dehydrogenation is observed. Only minor levels of superhydrogenation appear at high extinction due to the finite cloud size assumption. In terms of ionization, we see a larger contribution from $Z>0$ species at the surface of the cloud $(\sim 74 \%)$ compared to coronene and circumcoronene derivatives. There is no significant contribution from $Z=3$ nor $Z=4$ species (trications contribute in only $0.9 \%$ at $A_{V}=0$ ).

\section{Appendix E: Fiducial collision rates}

For neutral species we have considered a fiducial collision rate $k_{\text {col }}=\sigma_{\text {col }} \times v_{\text {th }} \times n(\mathrm{H})$, where $\sigma_{\text {col }}$ corresponds to the geometrical cross section of the parent molecule, and $v_{\text {th }}$ corresponds to the thermal velocity of the colliding $\mathrm{H}$ atoms. For anions we have considered a fiducial collision rate of $k_{\mathrm{col}}=7.8 \times 10^{-10} \times n(\mathrm{H})$, since we consider the $\mathrm{H}$-addition as an associative detachment reaction (see Sect. 2.4.4). For cations on the other hand, the fiducial collision rate adopted is of $k_{\mathrm{col}}=1.4 \times 10^{-10} \times n(\mathrm{H})$. When calculating the efficiency of $\mathrm{H}_{2}$ formation from direct $\mathrm{H}_{2}$-loss after photon absorption we multiply the fiducial rates by a 0.5 factor given that each photodissociation event removes $2 \mathrm{H}$ atoms from the molecule.

\section{Appendix F: Spectral variations}

\section{F.1. PAH emission spectra in the NW PDR of NGC 7023}

For coronene derivatives, the spectra are dominated by that of the completely dehydrogenated molecule. As listed in Table A.2, we do not have the intrinsic spectra of the anionic completely dehydrogenated coronene and thus, its contribution is not considered in the spectra shown in Fig. F.1. However we do not expect this to affect the resulting spectrum since the contribution from $\mathrm{C}_{24}^{-}$ increases smoothly from the surface of the cloud up to only a $7.4 \%$ at an $A_{V}=2$. In the case of circumcoronene, $\sim 34 \%$ of the emitting species are not considered in the spectrum at $A_{V}=$ 0 , where traces of the first partially dehydrogenated molecules are observed (e.g., $N_{\mathrm{H}}=17-12$ ). The spectra at $A_{V}=0.4$ to 1.2 on the other hand, are $>85 \%$ complete, while at $A_{V}=1.6$ and 2.0 the emission is complete to a $80 \%$. Since the contributions from partially dehydrogenated molecules are overall small, and they are not highly dehydrogenated, we expect the spectra to be representative of the actual emission. In the case of our largest species, circumcircumcoronene, all spectra are complete.

\section{F.2. $P A H$ emission spectra for different $G_{0} / n(H)$ ratios at $\mathrm{G}_{0}=10^{3}$}

In the case of coronene, the dominant species is the completely dehydrogenated molecule for most $G_{0} / n(\mathrm{H})$ ratios. Only 

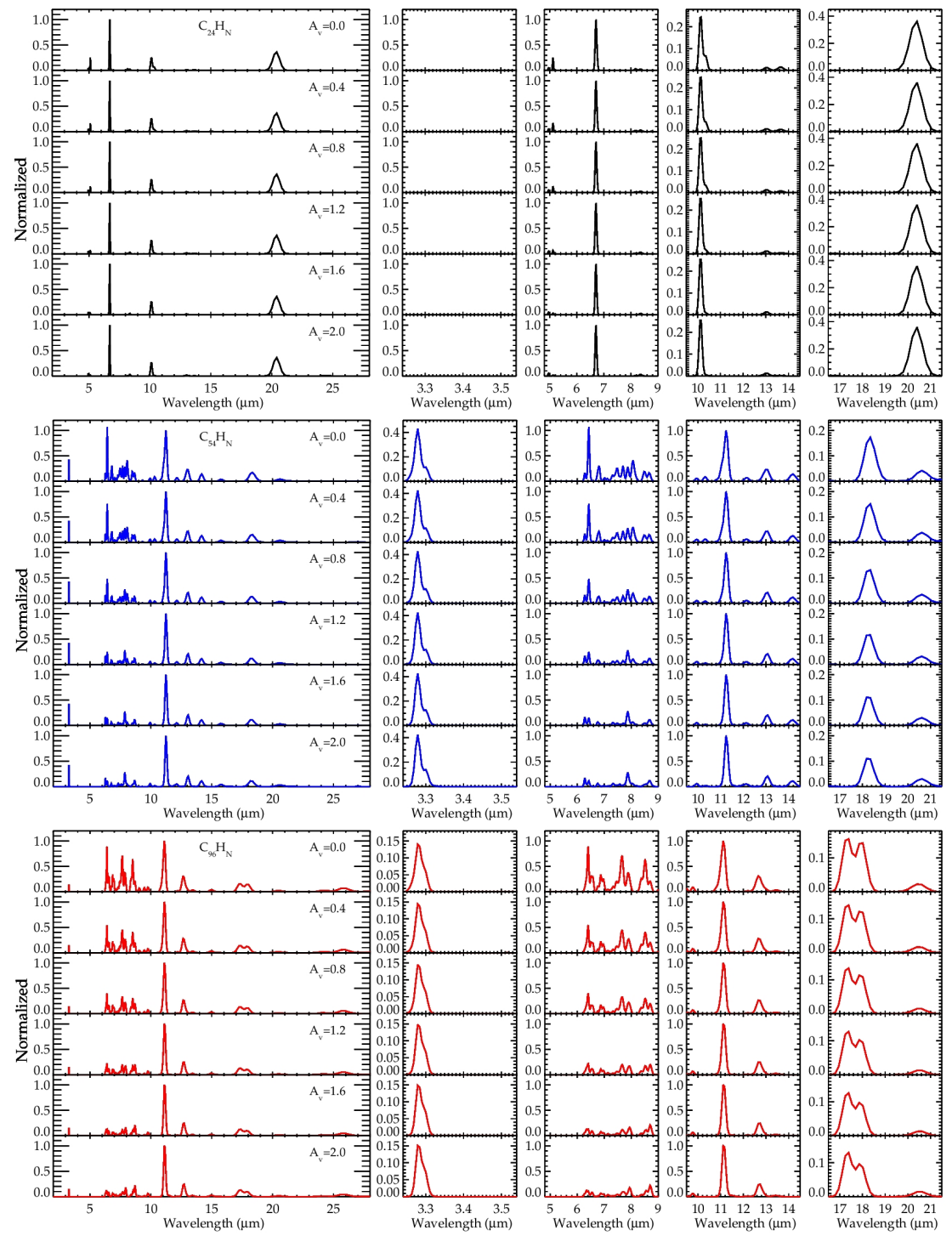

Fig. F.1. PAH emission spectra at different points inside the NW PDR of NGC 7023. For each species, the spectra have been derived by adding the contributions of all ionization and hydrogenation states, from the abundances presented in Appendix D. The results for $\mathrm{C}_{24} \mathrm{H}_{12}$ derivatives are shown in black, while the spectra for $\mathrm{C}_{54} \mathrm{H}_{18}$ and $\mathrm{C}_{96} \mathrm{H}_{24}$ species are shown in blue and red, respectively. The spectra have been conveniently normalized to the peak intensity of the $6.7 \mu \mathrm{m}$ band for $\mathrm{C}_{24} \mathrm{H}_{12}$ derivatives, and to the $11.2 \mu \mathrm{m}$ band for the larger species.

at $G_{0} / n(\mathrm{H}) \leq 0.01$ partially dehydrogenated derivatives in neutral and anionic form become more relevant. The spectrum at $G_{0} / n(\mathrm{H})=0.01$ is missing the contribution from $24 \%$ of the emitting species mostly due to $\mathrm{C}_{24}^{-}$, while the spectrum at $G_{0} / n(\mathrm{H})=$ 0.001 is missing $81.7 \%$ due to partially dehydrogenated neutrals and anions. The spectrum of normally hydrogenated coronene is never really important (even at a $G_{0} / n(\mathrm{H})=0.001 \mathrm{C}_{24} \mathrm{H}_{12}$ contributes at a $1 \%$ level only). The same applies for superhydrogenated species, which barely appear at $G_{0} / n(\mathrm{H})=0.001$ contributing at a 5\% level. The spectrum shown in Fig. F.2 is then mainly due to normal-to-superhydrogenated species and not from the partially dehydrogenated molecules, whose contribution would dominate the emission if they were available.

For circumcoronene, the dominant species at $G_{0} / n(\mathrm{H})=100$ is $\mathrm{C}_{54}^{+2}$ in $83 \%$ for which we do not have the IR spectrum. Therefore, the emission shown in Fig. F.2 corresponds mainly to that of $\mathrm{C}_{54}^{+}$, that contributes only in $12 \%$ to the total abundance, with even smaller traces of $\mathrm{C}_{54} \mathrm{H}$. Since the spectra of dications is in general similar to that of cations, we expect the actual emission to resemble the one shown in Fig. F.2, except for a stronger $5 \mu \mathrm{m}$ band, typical from highly positively ionized completely dehydrogenated PAHs. The spectra at $G_{0} / n(\mathrm{H})$ $=10$ is missing the emission from $54 \%$ of the species due to partially dehydrogenated molecules in positively ionized states.
Going to lower $G_{0} / n(\mathrm{H})$ values, the spectra at $G_{0} / n(\mathrm{H})=0.1$ and 0.01 are 99 and $79 \%$ complete respectively. Both dominated by $\mathrm{C}_{54} \mathrm{H}_{18}$. The contribution from superhydrogenated species in this $G_{0} / n(\mathrm{H})$ range goes from $\sim 3$ to $35 \%$, which is clear from the inspection of the $3 \mu \mathrm{m}$ region. The spectra at $G_{0} / n(\mathrm{H})=0.001$ is missing $97.5 \%$ of the emitting species due to the highly superhydrogenated molecules in anionic and neutral states. Thus the emission presented in the Figure corresponds only to the first superhydrogenated molecules for which we have the spectra.

Regarding circumcircumcoronene, the spectra are complete down to a $G_{0} / n(\mathrm{H})=0.1$, and show the variation in the ionization state of $\mathrm{C}_{96} \mathrm{H}_{24}$, from being mostly in $Z=3$ state to neutral. The spectrum at $G_{0} / n(\mathrm{H})=0.01$ is missing $19 \%$ of the emitting molecules due to highly superhydrogenated $Z=-1$ and $Z=0$ species, which then become the dominant species at $G_{0} / n(\mathrm{H})=0.001$. However these will not have a discernible footprint compared to that of the normally hydrogenated molecule which is still the dominating species: $\mathrm{C}_{96} \mathrm{H}_{24}^{-}$contributes in $8.8 \%$ and $\mathrm{C}_{96} \mathrm{H}_{24}^{0}$ in $68 \%$ at $G_{0} / n(\mathrm{H})=0.1$, and $26 \%$ and $21.6 \%$ respectively at $G_{0} / n(\mathrm{H})=0.01$. Also, going from $G_{0} / n(\mathrm{H})=0.1$ to 0.01 the abundance of superhydrogenated species - for which the spectra is considered in Fig. F.2 - increases by a factor of 10 , which is only clear in the $3 \mu \mathrm{m}$ spectral region, although the signal is rather weak. 

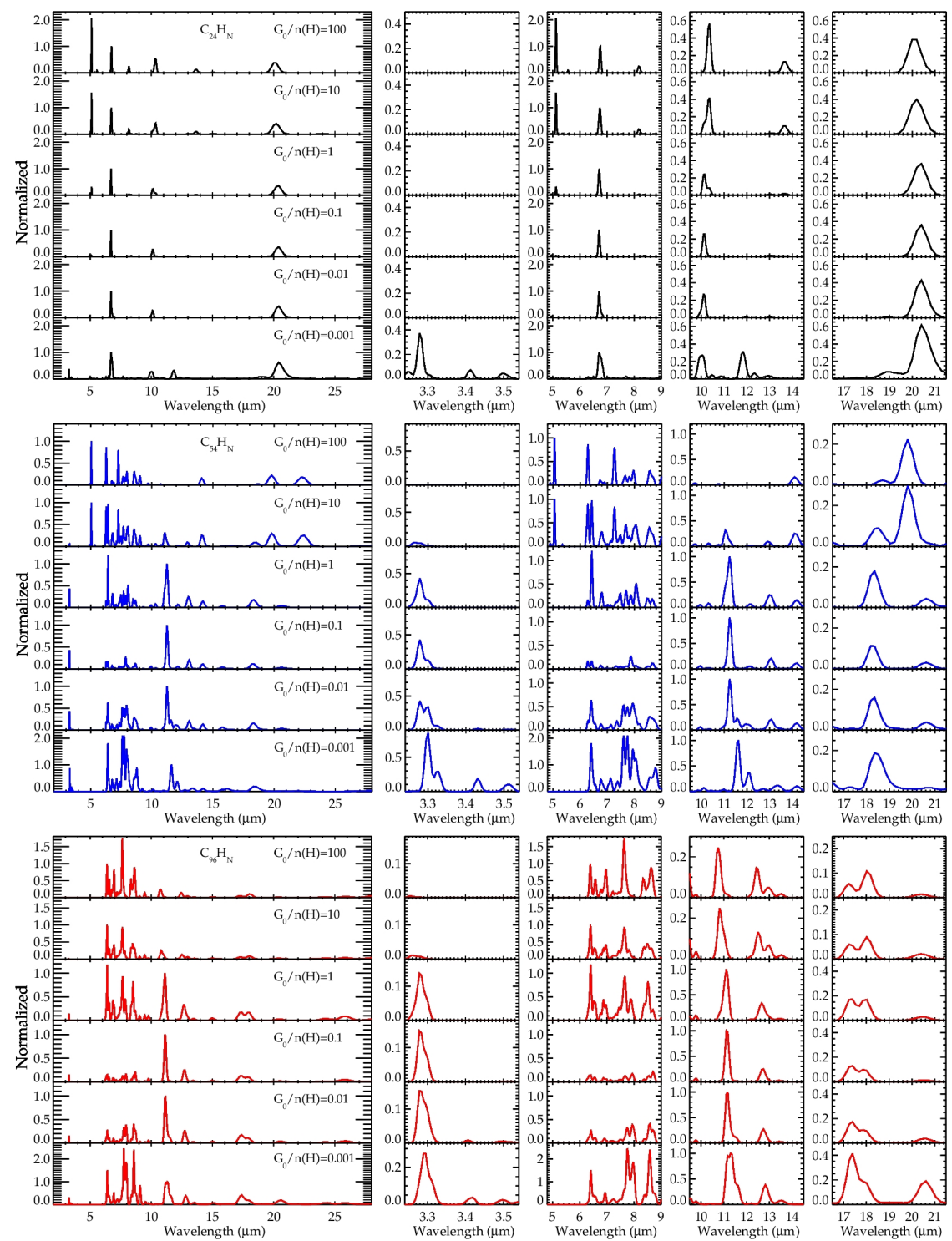

Fig. F.2. Emission spectra for different $G_{0} / n(\mathrm{H})$ ratios at $G_{0}=10^{3}$. The results for coronene derivatives are shown in black, while the spectra for $\mathrm{C}_{54} \mathrm{H}_{18}$ and $\mathrm{C}_{96} \mathrm{H}_{24}$ derivatives are shown in blue and red, respectively. The spectra have been conveniently normalized for a better comparison between consecutive $G_{0} / n(\mathrm{H})$ ratios. Given the unavailability of the intrinsic spectra for some of these molecules, the spectra at low $G_{0} / n(\mathrm{H})$ ratios are not complete due to the increasing abundance of highly superhydrogenated anionic species.

\section{F.3. Intrinsic spectra of different hydrogenation states of neutral coronene, circumcoronene and circumcircumcoronene molecules}

Figure F.3 shows a comparison of the IR spectra of different hydrogenation states of coronene, circumcoronene and circumcircumcoronene in the $3 \mu \mathrm{m}$ region. Here we see clearly the aromatic nature of the bands at 3.28-3.30 $\mu \mathrm{m}$ that disappear in the completely dehydrogenated molecules, and in the fully superhydrogenated molecule (e.g., $\mathrm{C}_{24} \mathrm{H}_{24}^{0}$ ). We have also checked this for other ionization states. The $3.5 \mu \mathrm{m}$ band is common to all superhydrogenated states of these species. This band is due to the $\mathrm{CH}$ (symmetric and asymmetric) stretching modes in a single $\mathrm{CH}_{2}$ group. In the case of $\mathrm{C}_{54} \mathrm{H}_{19}^{0}$ the bandat $3.5 \mu \mathrm{m}$ is very weak compared to the bands at $3.3 \mu \mathrm{m}$ (the peak intensity of the $3.28 \mu \mathrm{m}$ band is of $12 \times 10^{5} \mathrm{~cm}^{2} / \mathrm{mol}$, whereas the peak intensity of the band at $3.47 \mu \mathrm{m}$ is of $0.06 \times 10^{5} \mathrm{~cm}^{2} / \mathrm{mol}$ ). Thus it cannot be clearly seen with the scale used in this Figure. On the other hand, the band at $3.4 \mu \mathrm{m}$ appears from the second hydrogenation on, becoming particularly intense for the fully hydrogenated molecule in the case of coronene $\left(\mathrm{C}_{24} \mathrm{H}_{24}\right)$. This band is related to the $\mathrm{CH}$ stretches of the $\mathrm{CH}_{2}$ groups in the same (duo) rings. Superhydrogenated PAHs with extra $\mathrm{H}$ atoms in solo positions do not present this band. 
H. Andrews et al.: Hydrogenation and dehydrogenation of interstellar PAHs: Spectral characteristics and $\mathrm{H}_{2}$ formation
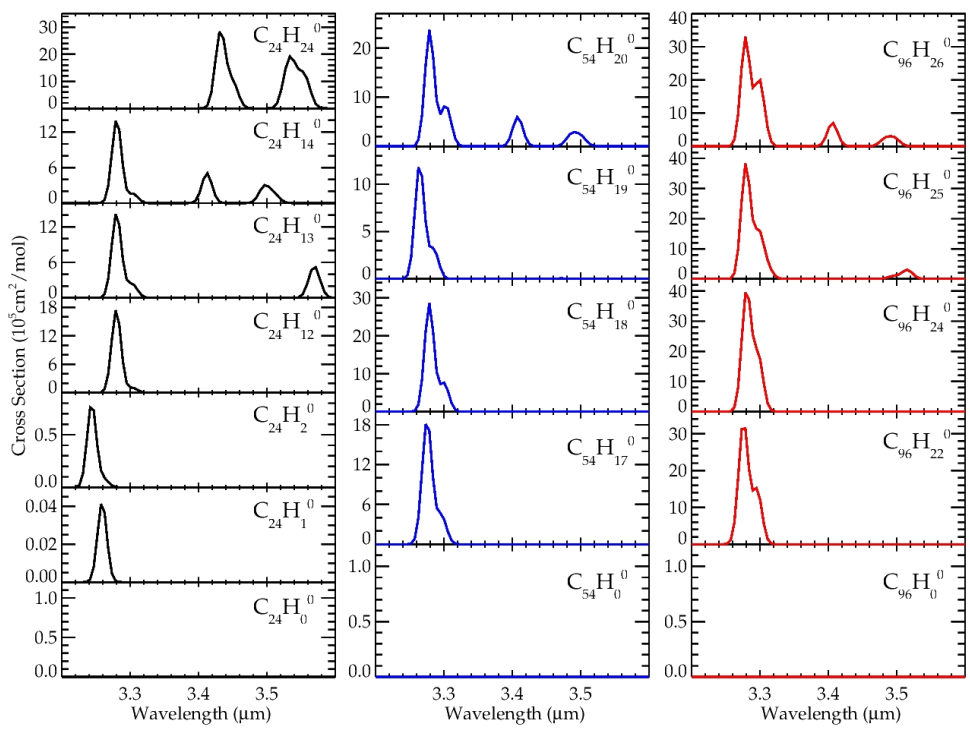

Fig. F.3. Intrinsic spectra of different hydrogenation states of the neutral coronene (left), circumcoronene (middle) and circumcircumcoronene (right panel) molecules. Only the $3 \mu \mathrm{m}$ region is presented. This spectral region is the one where differences in the hydrogenation state of PAHs can be clearly observed. Differences in the hydrogenation state (within a given PAH size and ionization state) are clear from the appearance of more features at 3.4 and $3.5 \mu \mathrm{m}$. 